\title{
MID-PACIFIC OCEANOGRAPHY
}

Part IV, Transequatorial Waters,

January-March, 1952

SPECIAL SCIENTIFIC REPORT-FISHERIES NO. 135 


\section{Explanatory Note}

The series embodies results of investigations, usually of restricted scope, intended to aid or direct management or utilization practices and as guides for administrative or legislative action. It is issued in limited quantities for the official use of Federal, State or cooperating Agencies and in processed form for economy and to avoid delay in publication. 
United States Department of the Interior, Douglas McKay. Secretary

Fish and Wildlife Service, John L. Farley, Director

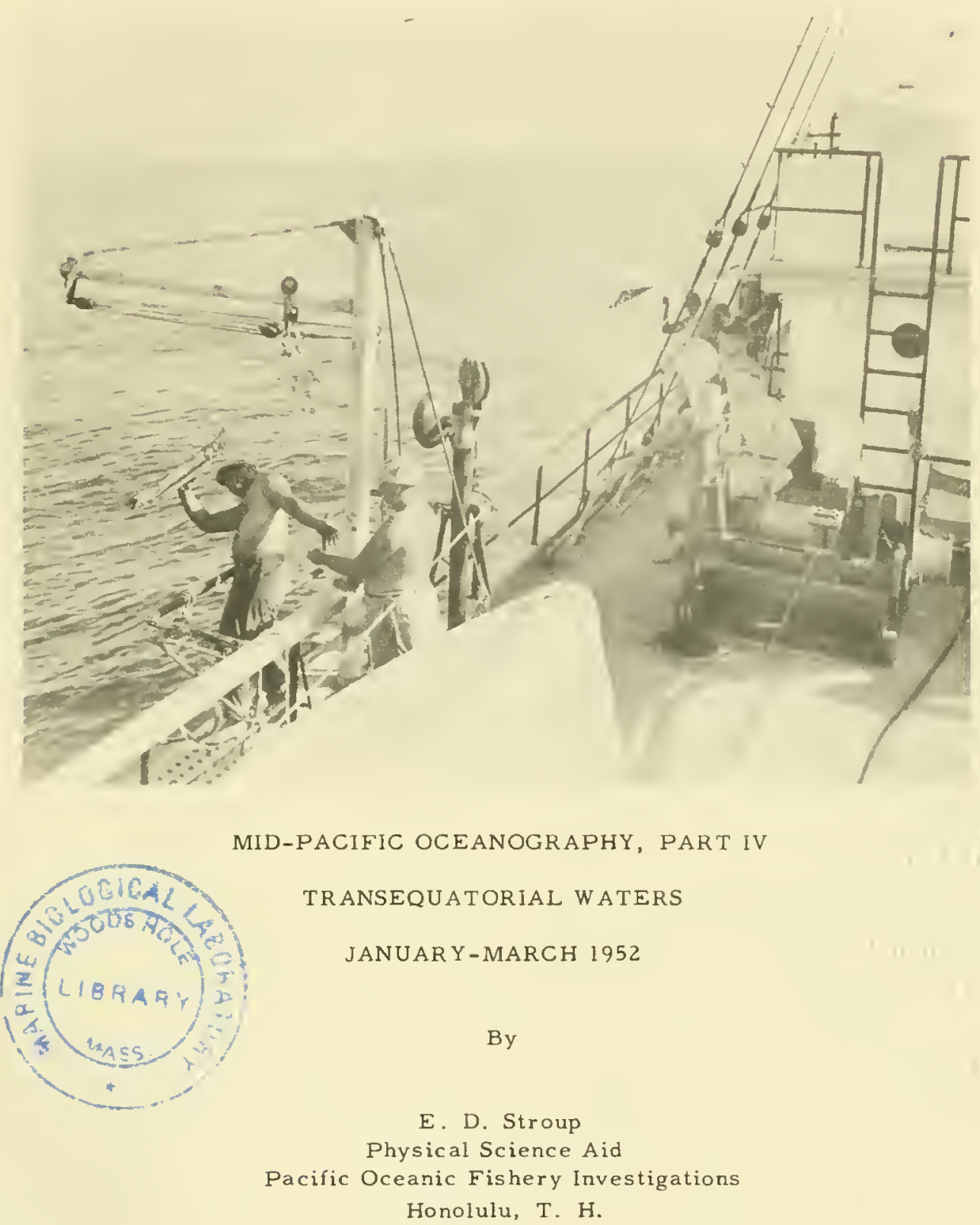

Special Scientific Report: Fisheries No. 135

W ASHINGTON: AUGUST 1954 



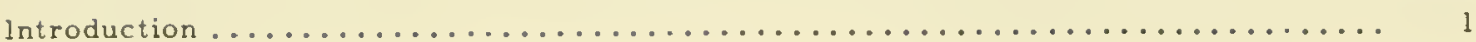

Vessel, equipment, and procedures $\ldots \ldots \ldots \ldots \ldots \ldots \ldots \ldots \ldots \ldots \ldots \ldots \ldots \ldots \ldots \ldots \ldots \ldots \ldots$

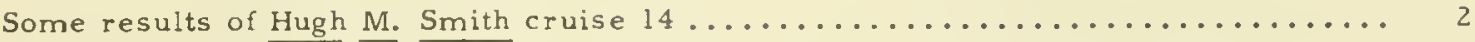

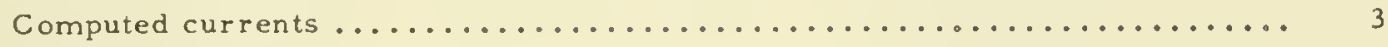

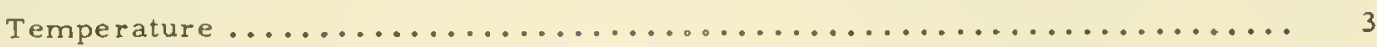

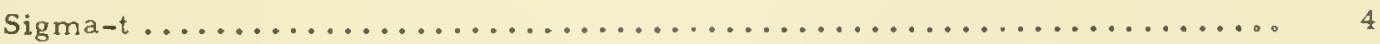

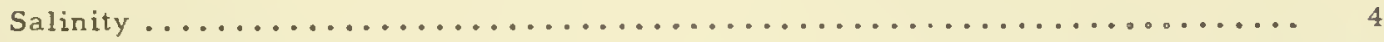

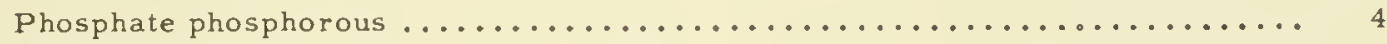

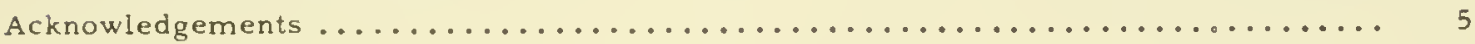

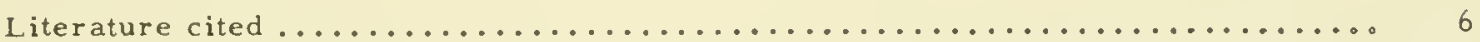

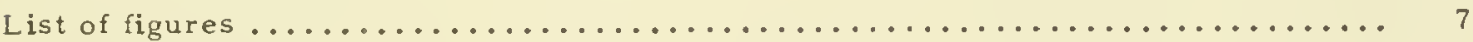

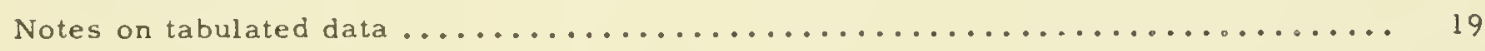



This report is concerned with cruise 14 of the $M / V$ Hugh $M$. Smith, the fifth in a series of equatorial oceanographic cruises by the Pacific Oceanic Fishery Investigations of the U. S. Fish and Wildlife Service. 1) The cruise was carried out in January and February 1952 in conjunction with longline tuna fishing in the same area on cruise 11 of the M/V John R. Manning; the results of the fishing have been described by Murphy and Shomura ( 1953 ). $\bar{T}$ he purpose of these simultaneous operations was to help relate the occurrence of tunas to the oceanography of the upper layers in the equatorial Pacific between $155^{\circ} \mathrm{W}$. and $180^{\circ}$ longitude.

Measurements of surface and subsurface temperature, salinity, and inorganic phosphate as well as plankton collections were made during Smith cruise 14 . The purpose of this report is to present the tabulated station data and various vertical sections, with minimum analys is of results. There are, in addition, brief descriptions of one of the smaller features of the area. The results of the plankton collections will appear in later reports.

\section{Vessel, Equipment, and Procedures}

The shipboard equipment was essentially as described by Cromwe!1 (1951), except that the hydrographic winch and work platform were raised to a safer and more workable location on the boat deck, about 6 meters above the sea surface (see frontispiece). The main-deck laboratories were also redesigned for greater convenience. The methods of collection and processing of the raw data have been described in detail (Cromwell 1951, 1954).

The treatment of the observed data from Smith cruise 14 leading to their ultimate presentation as interpolated data and vertical sections differs somewhat from the previous cruises. Smith cruises 5 and 8 (Cromwell 1954) and 11 (Austin 1954) were presented using modifications of the methods proposed by Montgomery in his evaluation of a Smith cruise 2 section (Montgomery 1954). The procedures used in the analysis of Smith cruise 14 are, in effect, an extension of Montgomery's method, embodying, in addition to the methods he employed, several further details suggested in his paper. An outline of the method of analysis will be given here; reference to the reports above (especially Montgomery 1954) should be made for detailed discussion and theoretical consideration.

The basic representation of each oceanographic station was made on a graph embodying a temperature scale vs. scales of depth, salinity, and phosphate phosphorous, with lines of constant sigma-t corresponding to the temperature and salinity arguments also included on the graph (fig. 1). Temperature-depth curves for each station were first drawn, passing through the values obtained from the reversing thermometers but utilizing the shape of the station bathythermograph (BT) trace to define detail in the upper 270 meters. Temperature-salinity and temperature-phosphate curves (station "characteristic curves") were then drawn. Values from adjacent stations, and in many cases from stations two or three removed on the same longitude, assisted in construction of the curves between observed points; this was readily accomplished by superposing the station graphs on a light table during the drawing. The necessity for this procedure is evident in an examination of the vertical sections (see below). The depths sampled by the Nansen bottles (entered as dots on the sections) necessarily often miss some of the major features of the distributions, especially in the region of the thermscline, but by utilizing adjacent-station values the continuity of these features is not destroyed. The variations in these characteristic curves with latitude were made as regular as possible, and, so far as the observed data permitted, maxima and minima were located on the same respective values of sigma-t from station to station.

1/ Cortain results of the previous cruises in this series appear in reports by Cromwell (1951, 1953, 1954), Austin (1954), and Montgomery (1954). 
Depths of sigma-t, salinity, and phosphate phosphorous at the desired isopleth values were taken from each station graph by referring to the common temperature scale and the temperature-depth curve, and each of these properties was plotted on a meridional section. Temperature sections to the same scales of latitude and depth were drawn for each station line of the cruise from the complete BT data (these were used in the analysis but are omitted here in favor of temperature sections with greater vertical exaggeration). The plots of sigma-t were then placed over the temperature sections on a light table and the isopleths were drawn, allowing the grosser detail of the temperature sections to influence the sigma-t sections between stations. The salinity and phosphate phosphorous plots were next drawn over the sigma-t sections in a similar fashion, with their isopleths made as nearly as possible parallel to the isopleths of sigma-t. In view of the extensive adjusting of the characteristic curves to obtain continuity between stations, the interpolated values of sigma-t, salinity, and phosphate phosphorous plotted on the sections were followed exactly during the drawing.

Values of temperature and salinity at standard depths were taken from the station graphs for use in the computation of geostrophic currents. The values of sigma-t tabulated at standard depths were computed from these temperature and salinity values, rather than read directly from the station graphs.

The most evident new procedure, that of allowing the BT temperature sections to influence construction of the sigma-t (and thus salinity and phosphate) sections, has been instituted to achieve consistency between the distributions of temperature and density, and internal consistency in the method of analysis. It is certainly not argued that all the detail thus introduced into the density distribution is meaningful from every point of view, but a more accurate presentation of the observed field of density is obtained than by merely drawing smooth curves through the station points. As a practical consideration, it is a great deal easier to include all the larger detail than to set up any arbitrary criterion for smoothing which would then have to be invoked continuously during the drawing of a section.

The method of mutual adjustment of the station characteristic curves is based on the fact that the ocean is continuous; with the present sampling methods this assistance between stations probably gives a closer representation of actuality than methods treating each station individually. The location of the maxima and minima of these curves on continuous values of sigma-t from station to station, and the subsequent drawing of the isopleths of the salinity and phosphate sections parallel to the isopleths of sigma-t, are based on the consideration that flow in the ocean normally takes place along surfaces of constant potential density, which are closely approximated by surfaces of constant sigma $-t$, and that mixing of by far the greatest magnitude takes place along these same surfaces.

\section{Some Results of Hugh M. Smith Cruise 14}

The station pattern for Smith cruise 14 appears in figure 2. The stations along $155^{\circ} \mathrm{W}$. longitude consisted of casts to at least 1,000 meters; to permit greater definition of detail in the region of interest the other two lines of stations were mostly limited to the upper 700 meters. Figures 3 to 7 , describing the section at $155^{\circ} \mathrm{W}$. longitude are in the sequence: dynamic topography, geostrophic currents, temperature, sigma-t, salinity. The second series of figures ( 8 to 11 ), describing the section at $180^{\circ}$ longitude and the third (12 to 15 ), describing the section at $169^{\circ} \mathrm{W}$. longitude are in the sequence: temperature, sigma-t, salinity, phosphate. Figure 16 presents temperature sections on $155^{\circ} \mathrm{W} .169^{\circ} \mathrm{W}$. and $180^{\circ}$ longitudes, taken on the fishing station lines of John R. Manning cruise 11 .

The numbers and positions of the stations are indicated on each section. For each variable except temperature, the location of every observation carried in the tabulated data is indicated by a spot on the vertical section in question. When there was serious doubt regarding the accuracy of an observation, it was not used in constructing the vertical section, and a footnote to this effect was entered in the tabulated data. Observations unquestionably in error were dropped from the tabulations. 
To make the relations between the different fields more readily apparent, selected isopleths of sigma-t have been plotted as dashed lines directly on the vertical sections of salinity and phosphate and on the section of computed currents. The two shallower isopleths roughly define the upper and lower boundaries of the strong density gradient in the thermocline.

Comparison of the temperature sections from Smith cruise 14 and Manning cruise 11 indicates that changes in the minor features of the various distributions may be quite rapid; this has been verified by the results of a subsequent cruise (Smith cruise 15, Austin MS.). For this reason no attempt has been made to plot any form of horizontal distribution over the area covered by Smith cruise 14.

It should be noted that the winds, atypically, were predominantly north of east over the entire area during the period of the cruise (wind data are listed for each station in the tabulated data).

Computed currents--As indicated above, only the stations along $155^{\circ} \mathrm{W}$. longitude were of sufficient depth to permit computation of geostrophic currents relative to a $1,000-m e t e r$ level of no motion (except for three stations on $180^{\circ}$ longitude, see below). The smoothed dynamic topographies of selected isobaric surfaces with respect to the 1,000-decibar surface are shown in figure 3; the computed dynamic heights at each station are carried as dots. Owing to equipment malfunctions the observations at $0^{\circ} 54^{\prime} \mathrm{N}$. latitude and $3^{\circ} 00^{\prime} \mathrm{S}$. latitude were too shallow to permit computation relative to this surface. From these smoothed topographies the currents shown in figure 4 were computed. The computation of currents becomes highly uncertain, and is not attempted, within $3^{\circ}$ of the Equator.

In the surface layer, the Equatorial Countercurrent is found from the northernmost station at $8^{\circ} \mathrm{N}$. latitude to the southern boundary at $4^{\circ} \mathrm{N}$. latitude; the Smith temperature section on this longitude (see below) indicates the northern boundary at approximately $9^{\circ} 30^{\prime} \mathrm{N}$. latitude. The westerly South Equatorial Current is found south of $4^{\circ} \mathrm{N}$. latitude.

The three southernmost stations of the $180^{\circ}$ longitude line reached 1,000 meters, and computation of dynamic heights of isobaric surfaces relative to this level have been carried out; these are carried in the tabulated data but not in a figure, and no currents have been computed. Examination of the data indicates that the currents in the surface layer were variable and perhaps partly easterly.

Temperature--Temperature sections from the BT data appear in figures $5,8,12$, and 16. As mentioned above, in order to reveal detail these sections were constructed with greater vertical exaggeration than those of the other variables. The BT casts from which the Smith sections were drawn were taken every 10 miles between stations, and every 30 miles along $155^{\circ} \mathrm{W}$. longitude north of $8^{\circ} \mathrm{N}$. latitude. Two casts were taken at each station; the one nearest in time to the hydrographic cast was plotted in each case. The Manning sections are drawn from casts made on the fishing stations only. The Manning fished one station per day; the date of the first station on each line is given in the figure caption.

On each section the downward slope of the thermocline away from the Equator to north and south (as distinguished from the pronounced arching of the shallower isotherms at the Equator) is evidence of the distribution of mass associated with the South Equatorial Current, and the northward rise starting a few degrees north of the Equator is associated with the easterly flow of the Equatorial Countercurrent. Only the Smith section along $155^{\circ} \mathrm{W}$. longitude extends beyond this into the North Equatorial Current, indicated by a second northward deepening. The southward rise of the isotherms at the southern end of the $180^{\circ}$ longitude line is reflected in the weakening of the westerly flow indicated here by the computed dynamic heights of isobaric surfaces. These gross "topographic" features, associated primarily with zonal currents, are reflected in the distributions of each of the variables. In the surface layer, lower temperatures at the Equator are evidence of the addition of water from somewhat greater depths. 
As mentioned above, a comparison of the Smith and Manning sections indicates the possibility of quite considerable changes in detail in a relatively short time, although the basic features remain distinct.

Surface temperatures are indicated at the top of each temperature section. These are often influenced by shallow diurnal effects, as at $3^{\circ} 15^{\prime} \mathrm{N}$. latitude, $180^{\circ}$ longitude (fig. 8 ); no attempt was made to include these temporary variations in the vertical temperature sections.

Several of the details of the temperature field, such as the reduction of the vertical temperature gradient at the Equator and the presence of stable inversions in the thermocline near the Equator, are described in reports of this series (Cromwell 1954, Austin Mj.). Both of these features are seen on each of the Smith cruise 14 temperature sections. A detail not so far described is the persistent, relatively "sharp" bottom of the main thermocline at about $2^{\circ}$ $3^{\circ}$ N. latitude. Figure 17 presents BT traces taken near these latitudes on various longitudes during several cruises. The traces best illustrating the phenomenon were chosen from a meridional series in each case, demonstrating a remarkable consistency in position; it has noreover never been completely absent. Figure 18 gives the detailed temperature structure on the oblique section marked on figure 2. The most evident "sharp" bottom of the thermocline is seen at $2^{\circ} 06^{\prime} \mathrm{N}$. latitude, $175^{\circ} 04^{\prime} \mathrm{W}$. Iongitude; this particular trace is second from the left in figure 17 .

Sigma-t--The distribution of sigma-t is shown in figures 6, 9, and 13. These figures are closely associated with, and share most of the details of, the temperature sections (see procedures). For an exception to this rather general rule, the surface layer decrease in density at the southern end of the $180^{\circ}$ longitude line is largely associated with a decrease in salinity. The expected slight east to west thickening of the light surface layer may be seen in a comparison of the sections.

There is a marked meridional change in the character of the water just beneath the main thermocline in approximately the $2^{\circ}-3^{\circ} \mathrm{N}$. latitude region discussed above; this feature is reflected in the sharp southerly increase in depth of the sigma-t isopleths 26.6 to 27.0 in this area. The effect of this distribution of density is evident in the plot of dynamic heights of isobaric surfaces, but at these latitudes the interpretation is extremely uncertain. Barnes et al. (1948, p. 872) describe what seems to be a similar case in the Marshall Islands area, with regard to a dip in the $10^{\circ} \mathrm{C}$. isotherm.

Salinity-- The vertical sections of the field of salinity are shown in figures 7,10 , and 14. The major features of the distribution are as described previously for the central Pacific (Cromwell 1951, 1953, 1954; Austin 1954; Montgomery 1954; Mao and Yoshida 1953). The similarity from section to section of Smith cruise 14 is self-evident.

The detailed vertical distribution of salinity in the region of $2^{\circ}-3^{\circ} \mathrm{N}$. latitude is of interest and is not readily evident from the sections. Figure 1, illustrating the method of representing the oceanographic stations is taken from Smith cruise 14,5 tation 27 at $2^{\circ} 07^{\prime} \mathrm{N}$. latitude, $179^{\circ} 57^{\prime} \mathrm{W}$. longitude and shows this distribution. The sharp bottom of the thermocline seems to be a boundary between overlying water associated with a salinity minimum extending from the north, in the thermocline, and deeper water with a temperature-salinity relation identical with that of water to the south. The salinity decreases downward through the thermocline, increases sharply across the boundary, then decreases gradually again below; the relative maximum and minimum values lie close together vertically. With the isopleth values used, this feature is most evident on the $180^{\circ}$ longitude section. The increase in salinity across the boundary is often accompanied by a stable temperature inversion. Farther to the north the vertical transition below the salinity minimum is more gradual; toward the Equator the salinity minimum disappears into the very salty water extending into the area from the south.

Phosphate phosphorous -- The vertical sections of inorganic phosphate for longitudes $180^{\circ}$ and $169^{\circ} \mathrm{W}$. appear in figures 11 and 15 . On the $155^{\circ} \mathrm{W}$. longitude line the colorimeter 
broke down after the first five stations; data for these are tabulated but no figure was drawn. A relatively higher concentration of phosphate about the Equator in the surface layer. seen on the two sections included, is evidence of enrichment from below.

\section{Acknowledgements}

Many more persons than can be mentioned here share the creait for the successful completion of Smith cruise 14, and for the publication of this report. The field party personnel during the cruise comprised: Garth I. Murphy (field party chief), E. D. Stroup, H. Yuen, T. Hida, and T. Roseberry. Special thanks must go to Captain Ralph Johnson and the crew of the Hugh M. Smith for the utmost cooperation with the scientific field party. Ashore, Mary Lynne Godfrey was largely responsible for the processing of the data. Tamotsu Nakata drafted the figures.

T. S. Austin, T. Cromwell, and Dr. R. B. Montgomery gave invaluable assistance and encouragement in the many stages of preparation of the data, and during the writing of the report. 
AUSTIN, T.S.

1954. Mid-Pacific oceanography 1II, transequatorial waters, August-October 1951. U. S. Fish and Wildlife Serv., Spec. Sci. Rep.: Fish. No. 131. (In press).

MS. Mid-Pacific oceanography V, transequatorial waters, May-June 1952 , August 1952 .

BARNES, C. A., D. R. BUMPUS and J. LYMAN

1948. Ocean circulation in the Marshall islands area. Trans. Amer. Geophys. Union, vol. 29, No. 6, pp. $871-876$.

CROMWELL, T.

1951. Mid-Pacific oceanography. January-March 1950. U. S. Fish and Wildlife Serv.. Spec. Sci. Rep.: Fish. No. 54, 9 pp.

1953. Circulation in a meridional plane in the central equatorial Pacific, Jour. of Mar. Res., Vol. 12, No. 2, pp. 196-213.

1954. Mid-Pacific oceanography II, transequatorial waters, June-August 1950, JanuaryMarch 1951. U. S. Fish and Wildlife Serv., Spec. Sci. Rep.: Fish. No. 131. (In press).

MAO, HAN-LEE and K. YOSHIDA

1953. Physical oceanography in the Marshall islands area. Univ. of Calif., Scripps Inst. of Ocean., Ref. 53-27, April 15, 1953.

MONTGOMERY, R. B.

1954. Analys is of a Hugh M. Smith oceanographic section from Honolulu southward across the Equator. Accepted for publication in the Jour. of Mar. Res., Vol. 13, No. 1.

MURPHY, G. I. and R. S. SHOMURA

1953. Longline fishing for deep-swimming tunas in the central Pacific, January-June 1952. U. S. Fish and Wildlife Serv., Spec. Sci. Rep.: Fish. No. 108, 32 pp. 
Frontispiece: Hydrographic platform and winch, Hugh M. Smith cruise 14. Photo by G. I. Murphy.

1. Example of station data representation.

2. Station positions.

3. Dynamic topography, $155^{\circ} \mathrm{W}$.

4. Geostrophic currents, $155^{\circ} \mathrm{W}$.

5. Vertical section of temperature, $155^{\circ} \mathrm{W}$.

6. Vertical section of sigma-t, $155^{\circ} \mathrm{W}$.

7. Vertical section of salinity, $155^{\circ} \mathrm{w}$.

8. Vertical section of temperature, $180^{\circ}$.

9. Vertical section of sigma-t, $180^{\circ}$.

10. Vertical section of salinity, $180^{\circ}$.

11. Vertical section of phosphate, $180^{\circ}$.

12. Vertical section of temperature, $169^{\circ} \mathrm{W}$.

13. Vertical section of sigma-t, $169^{\circ} \mathrm{W}$.

14. Vertical section of salinity, $169^{\circ} \mathrm{W}$.

15. Vertical section of phosphate, $169^{\circ} \mathrm{W}$.

16. Vertical sections of temperature, John R. Manning cruise 11 .

17. BT traces showing "sharp" thermocline bottom.

18. BT section showing "sharp" thermocline bottom. 


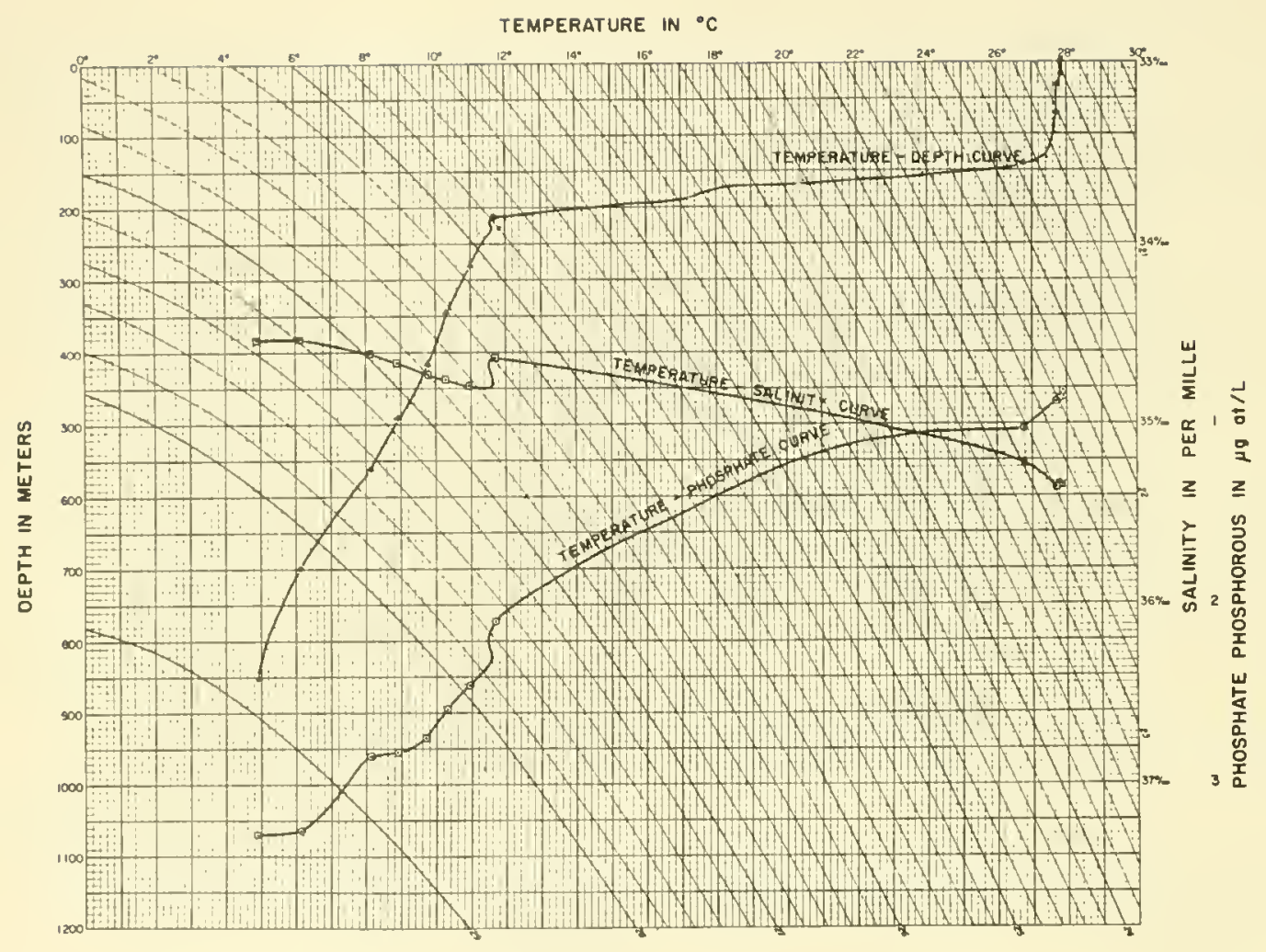

Fig. 1--(Revised from Cromwell 1954). Example of graph used to present temperature, salinity, and phosphate data for each station. Points under inverted $V$ represent reversing thermometer temperatures; plain points are taken from the station BT trace (both plotted against depth). Points in a square represent salinity, and those in a circle phosphate, both plotted against temperature.

Smith cruise 14 , station $27,2^{\circ} 07^{\prime} \mathrm{N}$. latitude, $179^{\circ} 57^{\prime} \mathrm{W}$. longitude, February 19, 1952, 1336 GCT. 


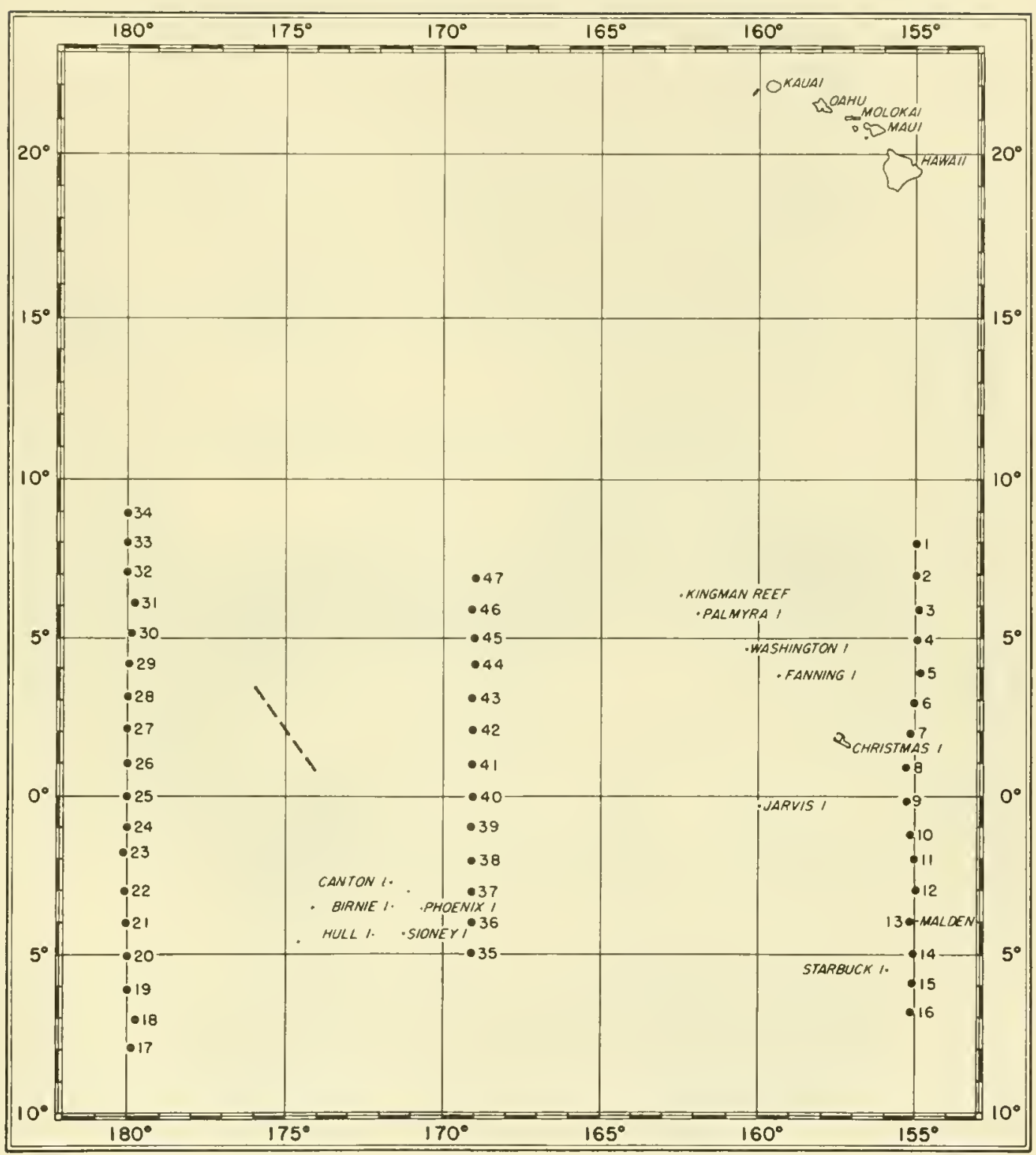

Fig. 2--Hydrographic station positions. Hugh M. Smith cruise 14, January-March 1952. Location of temperature section in figure 18 indicated by dashed line. 


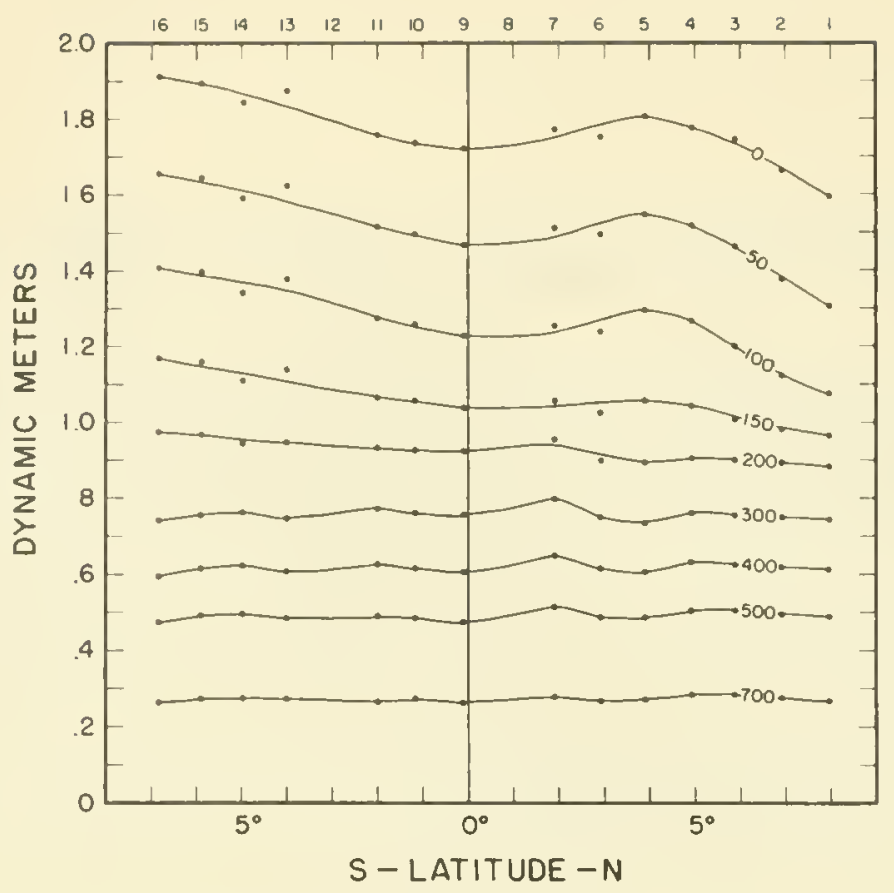

Fig. 3--Smoothed anomaly of topographies of isobaric surfaces relative to the 1,000-decibar surface. Hugh M. Smith cruise 14,

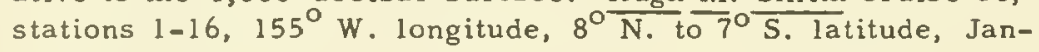
uary-February 1952. Points represent computed values.

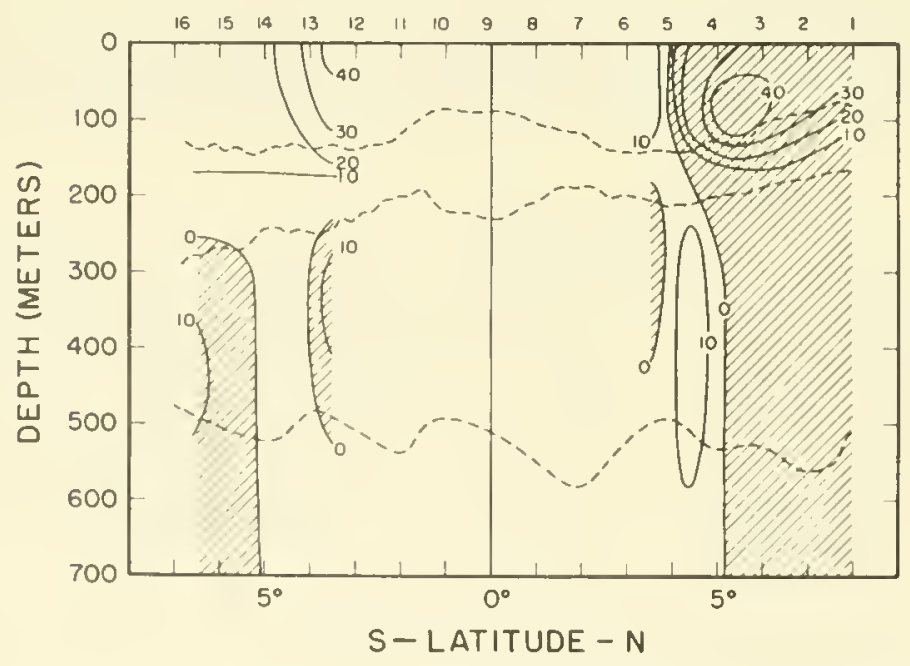

Fig. 4--Zonal component of geostrophic currents in centimeters per second. Hugh $M$. Smith cruise 14 , stations $1-16,155^{\circ} \mathrm{W}$.

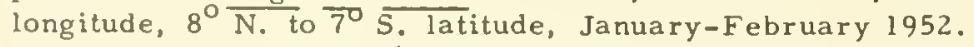
Plotting interval $10 \mathrm{~cm} / \mathrm{sec}$. East currents hatched. Isopleths of sigma-t $=23.2,26.4$ and 27.0 entered as dashed lines. 


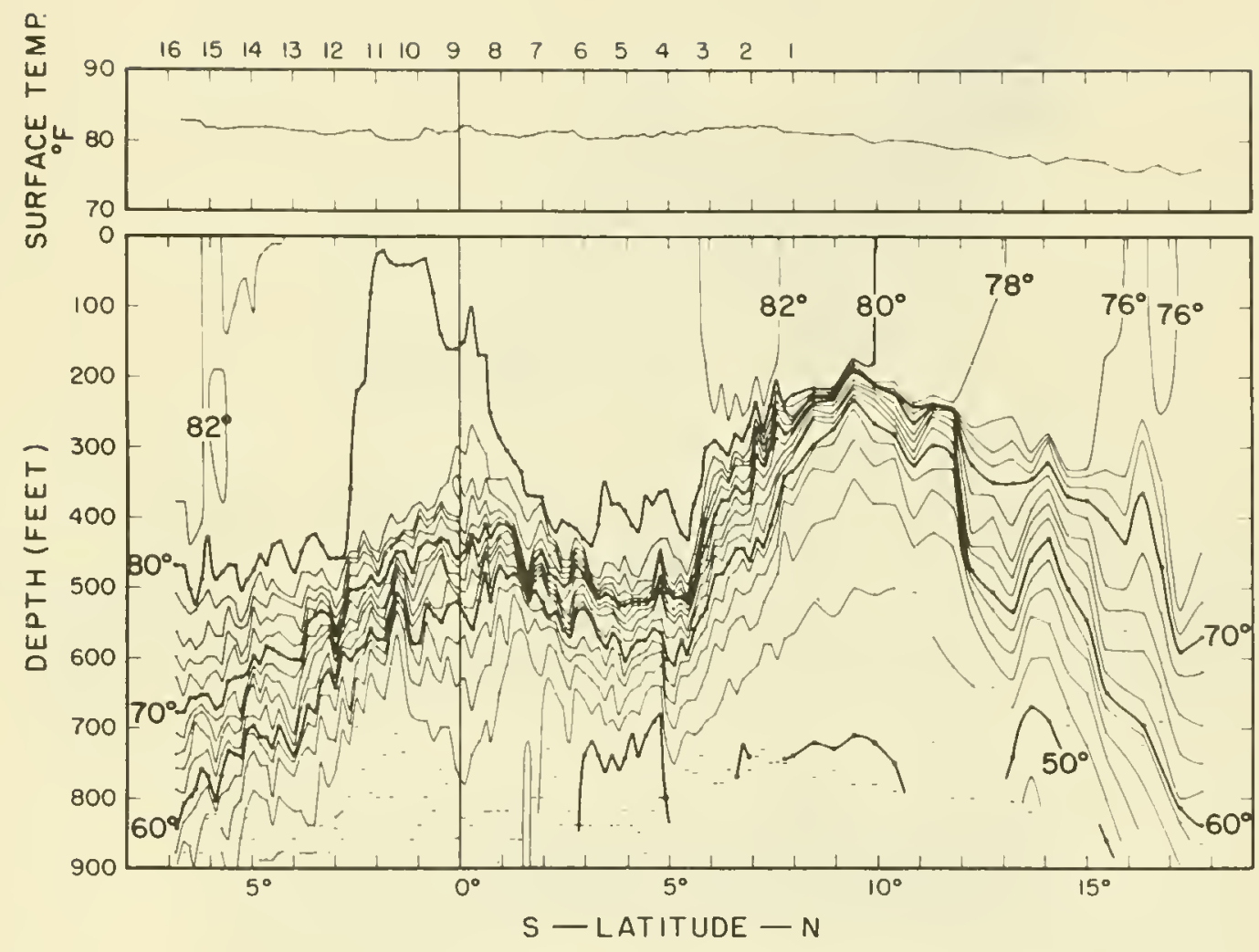

Fig. 5--Upper: surface temperatures by bucket thermometer in degrees F., measured with each BT cast. Lower: temperatures from BT data in degrees F. Hugh M. Smith cruise 14 , stations $1-16,155^{\circ} \mathrm{W}$. longitude,

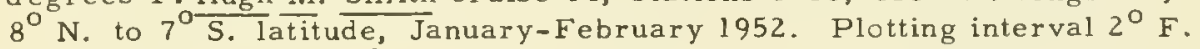
Horizontal dashes indicate depths of observations less than 900 feet. 


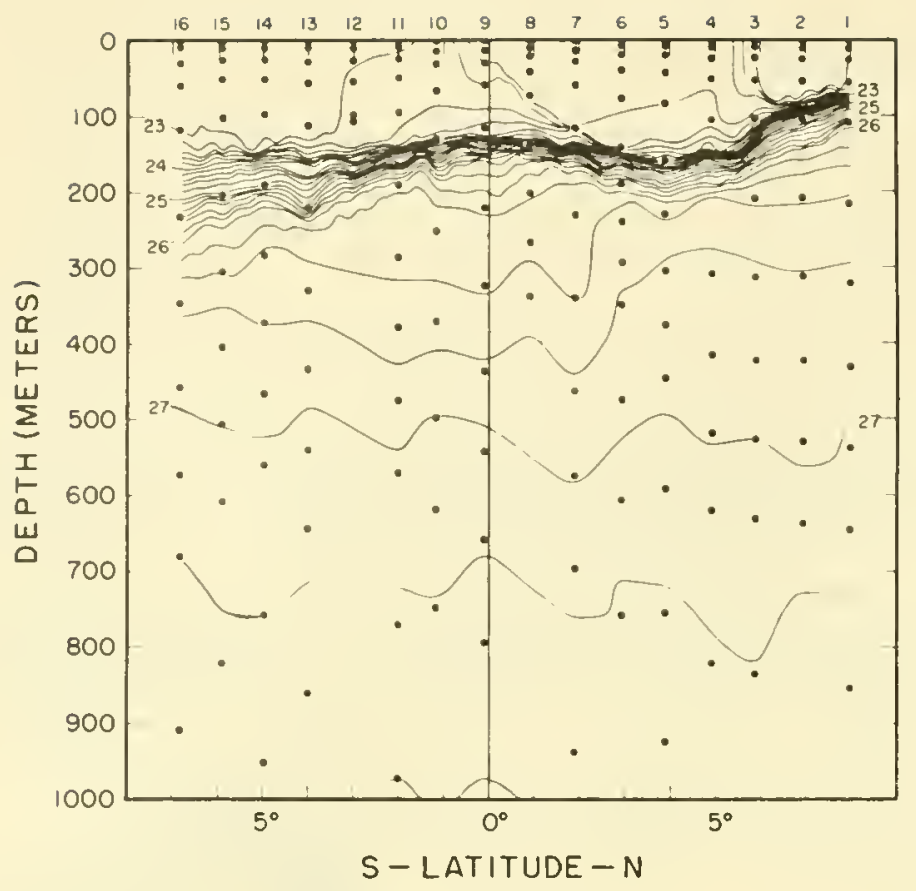

Fig. 6--Sigma-t in grams per liter. Hugh M. Smith cruise 14, stations $1-16,155^{\circ} \mathrm{W}$. longitude, $8^{\circ} \overline{\mathrm{N} \text {. to }} \overline{7}^{\circ} \mathrm{S}$. latitude, January February 1952. Plotting interval $0.2 \mathrm{~g} / \mathrm{l}$.

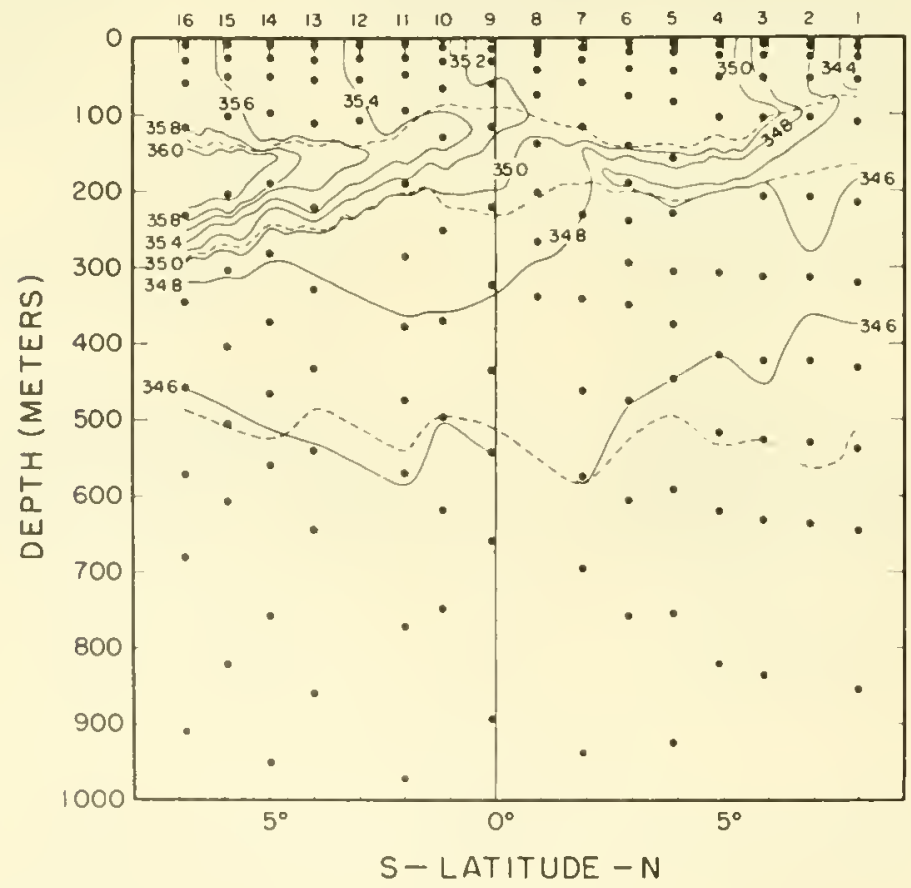

Fig. 7--Salinity in per mille. Hugh M. Smith cruise 14, stations

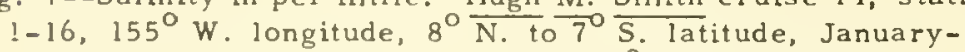
February 1952. Plotting interval $0.2 \%$. Isopleths of sigma-t $=23.2,26.4$ and 27.0 entered as dashed lines. 


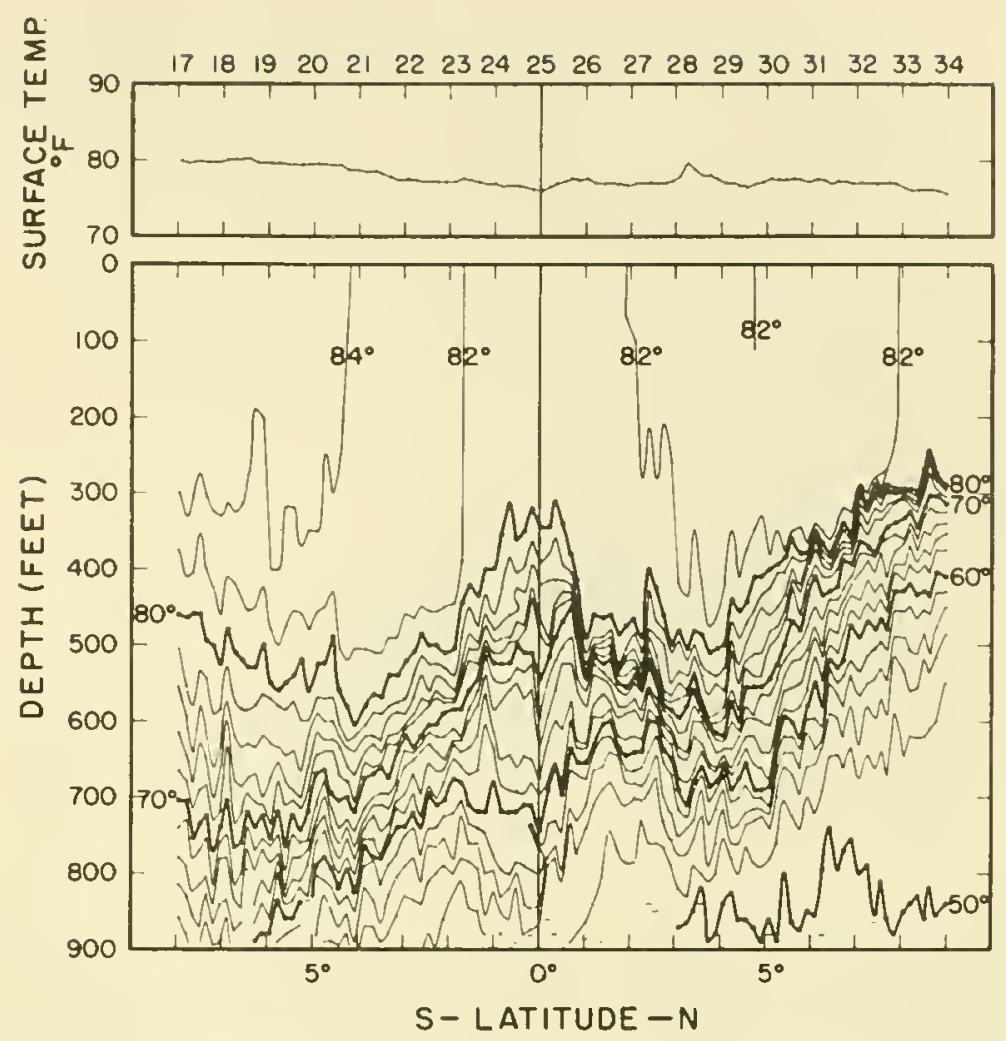

Fig. 8--Upper: surface temperatures by bucket thermometer in degrees F., measured with each BT cast. Lower: temperatures from BT data in degrees F. Hugh M. Smith cruise 14 , stations $\overline{17-34,} 180^{\circ}$ longitude, $8^{\circ} \mathrm{S}$. to $9^{\circ} \mathrm{N}$. latitude, February $\overline{1952}$. Plotting interval $2^{\circ} \mathrm{F}$. Horizontal dashes indicate depths of observations less than 900 feet.

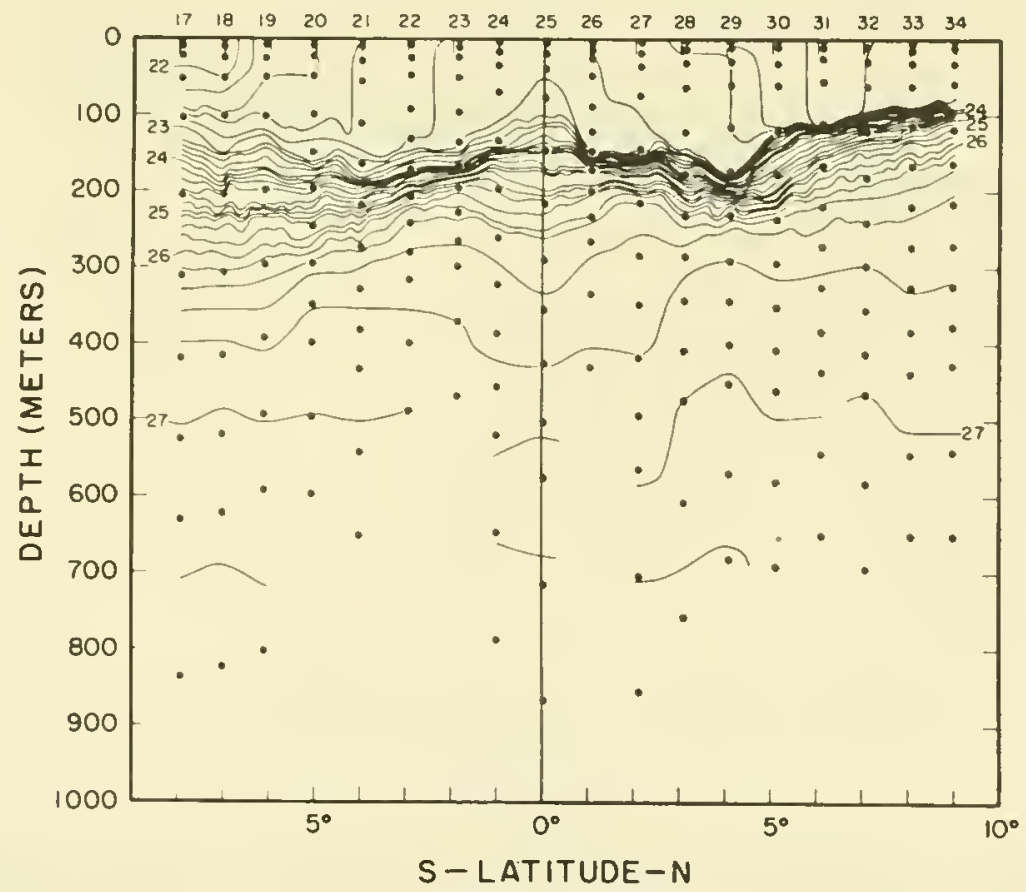

Fig. 9--Sigma-t in grams per liter. Hugh M. Smith cruise 14, stations 17-34, 180 longitude, $8^{\circ} \mathrm{S}$. to $9^{\circ} \mathrm{N}$. latitude, February 1952 . Plotting interval $0.2 \mathrm{~g} / \mathrm{l}$. 


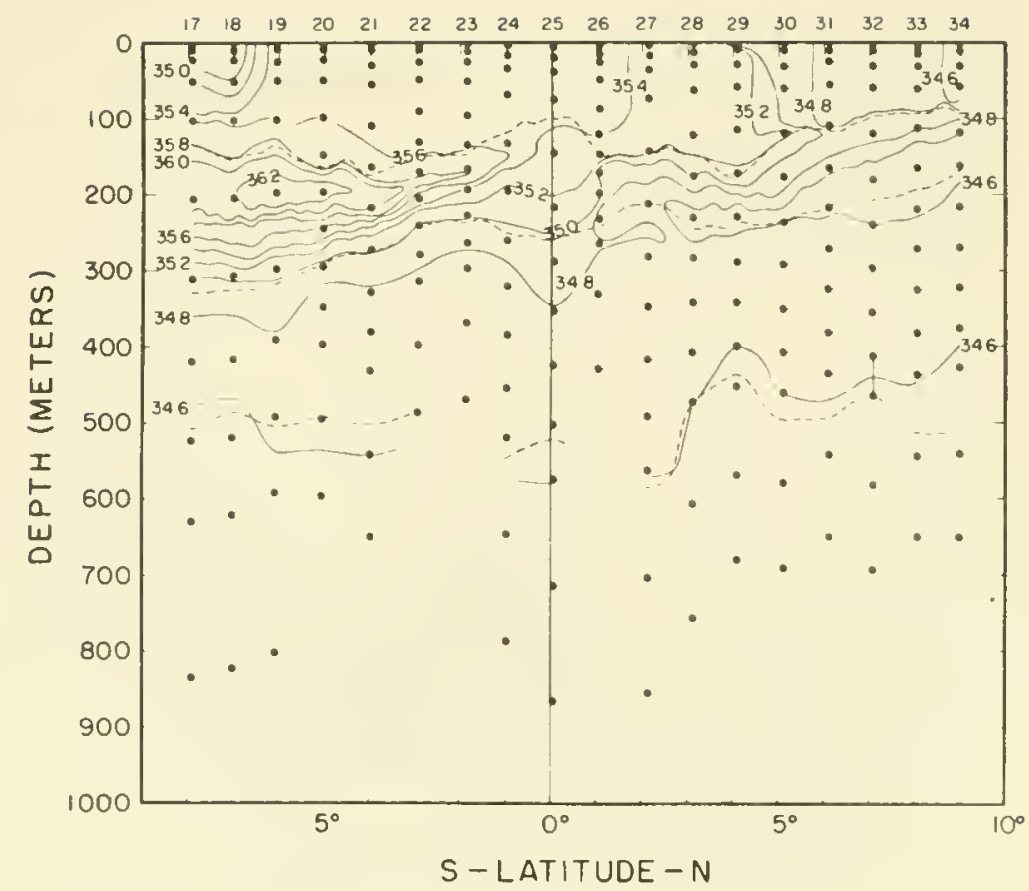

Fig. 10--Salinity in per mille. Hugh M. Smith cruise 14, stations 17-34, $180^{\circ}$ longitude, $8^{\circ}$ S. to $9^{\circ}$ N. latitude, February 1952. Plotting interval $0.2 \%$. Isopleths of sigma-t = 23.2, 26.4 and 27.0 entered as dashed lines. Solid vertical line indicates vertically isohaline water at the salinity of the intersecting isohaline.

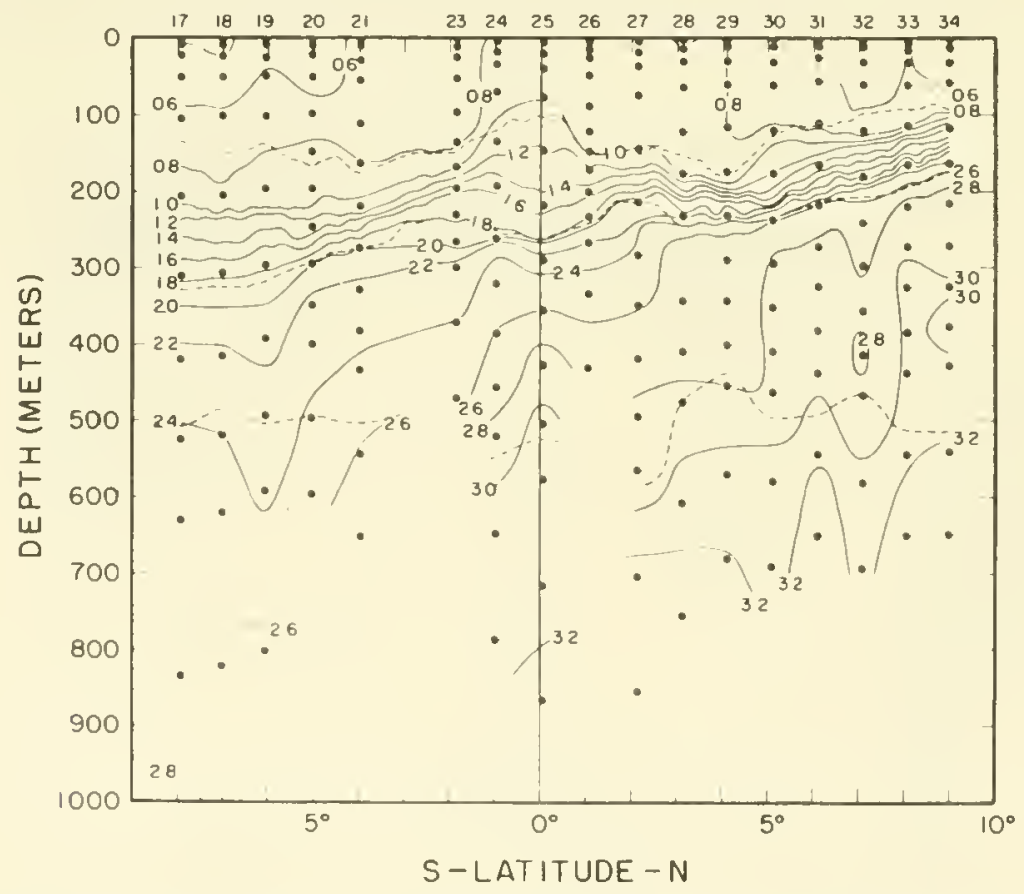

Fig. 11--Phosphate phosphorous in microgram-atoms per liter. Hugh M. Smith cruise 14, stations $17-34,180^{\circ}$ longitude, $8^{\circ} \mathrm{S}$. to $9^{\circ} \mathrm{N}$. latitude, February $19 \overline{52}$. Plotting interval $0.2 \mu g-a t / 1$. Is opleths of sigma-t $=23.2,26.4$ and 27.0 entered as dashed lines. 


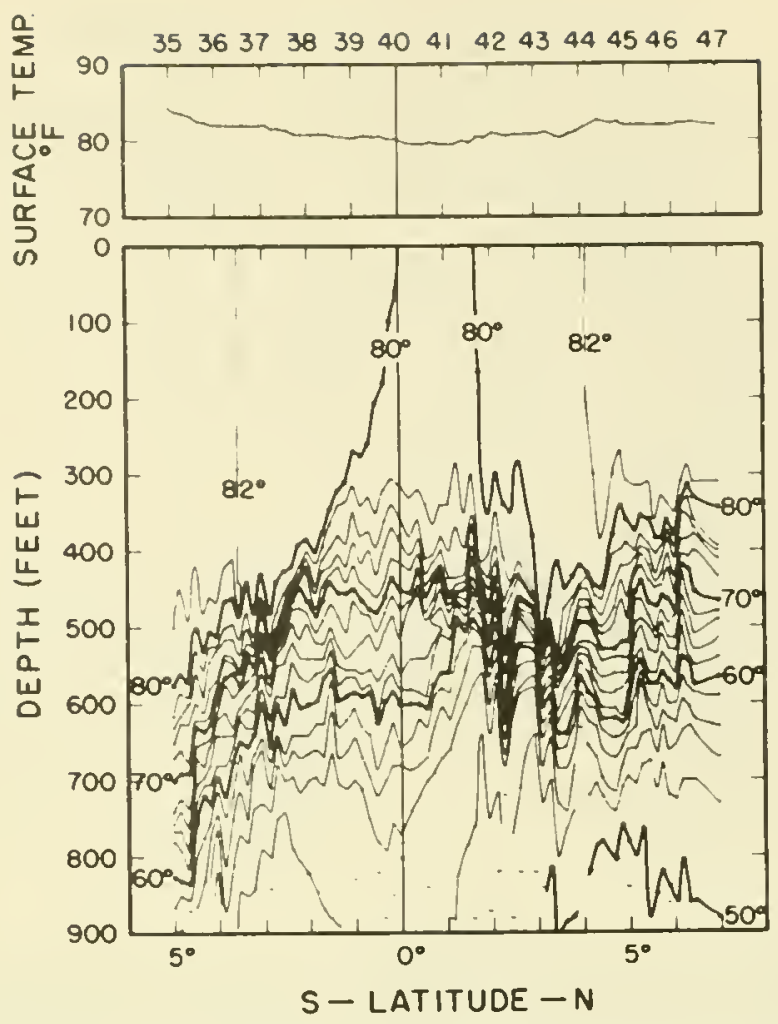

Fig. 12--Upper: surface temperatures by bucket thermometer in degrees F., measured with each BT cast. Lower: temperatures from BT data in degrees F. Hugh M. Smith cruise 14 , stations $35-47,169^{\circ} \mathrm{W}$. longitude, $5^{\circ} \mathrm{S}$. to $7^{\circ} \mathrm{N}$. latitude, March $\overline{1952 .}$ Plotting interval $2^{\circ} \mathrm{F}$. Horizontal dashes indicate depths of observations less than 900 feet.

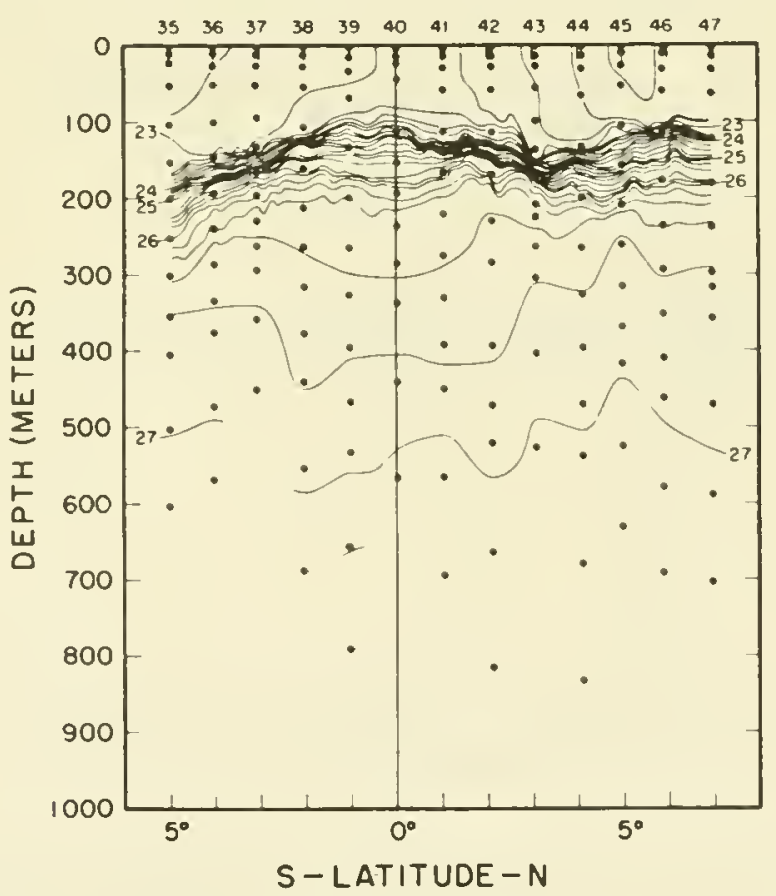

Fig. 13--Sigma-t in grams per liter. Hugh M. Smith cruise 14, stations $35-47,169^{\circ}$ W. longitude, $5^{\circ} \mathrm{S}$. to $7^{\circ} \mathrm{N}$. latitude, $\overline{\mathrm{Mar}} \overline{\mathrm{M}} 1952$. Plotting interval $0.2 \mathrm{~g} / \mathrm{l}$. 


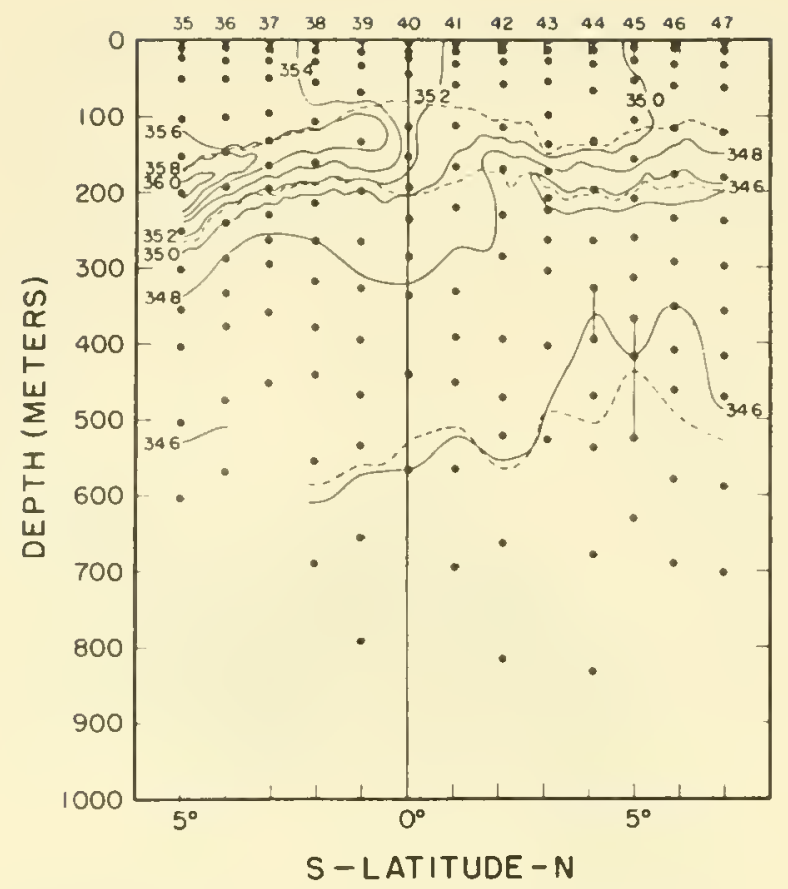

Fig. 14--Salinity in per mille. Hugh M. Smith cruise 14, stations 3547, $169^{\circ} \mathrm{W}$. longitude, $5^{\circ} \mathrm{S}$. to $7^{\circ} \overline{\mathrm{N}}$. latitude, March 1952. Plotting interval $0.2 \%$. Isopleths of sigma-t $=23.2,26.4$ and 27.0 entered as dashed lines. Solid vertical line indicates vertically isohaline water at the salinity of the intersecting isohaline.

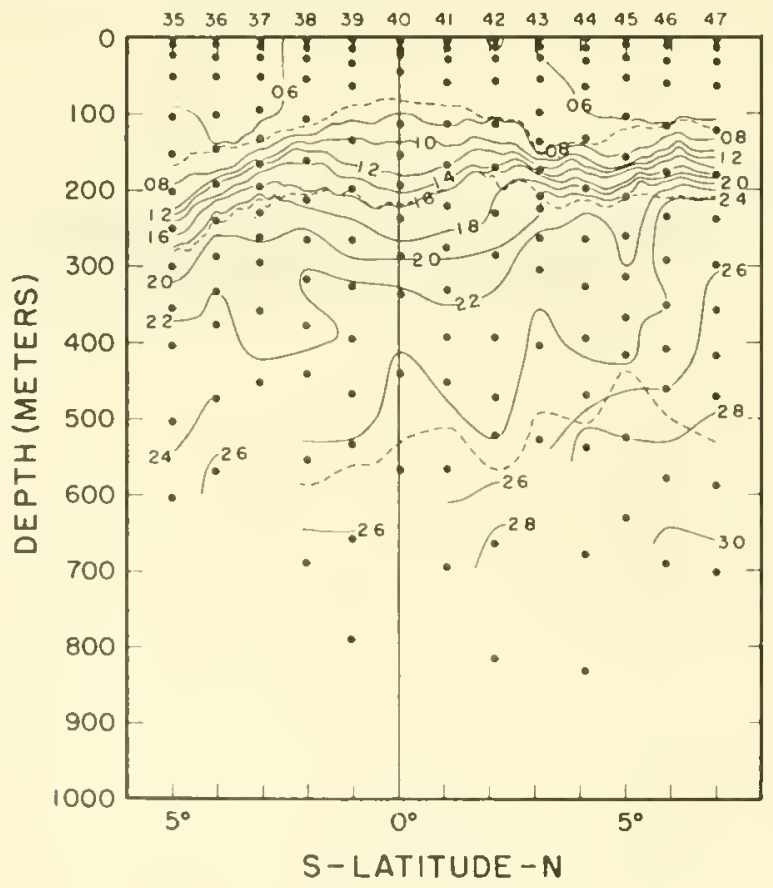

Fig. 15--Phosphate phosphorous in microgram-atoms per liter. Hugh M. Smith cruise 14 , stations $35-47,169^{\circ} \mathrm{W}$. longitude, $5^{\circ}$ S. to $7^{\sigma} \mathbf{N}$. latitude, March 1952. Plotting interval $0.2 \mu \mathrm{g}-\mathrm{at} / \mathrm{l}$. Isopleths of sigma-t $=23.2,26.4$, and 27.0 entered as dashed lines. 

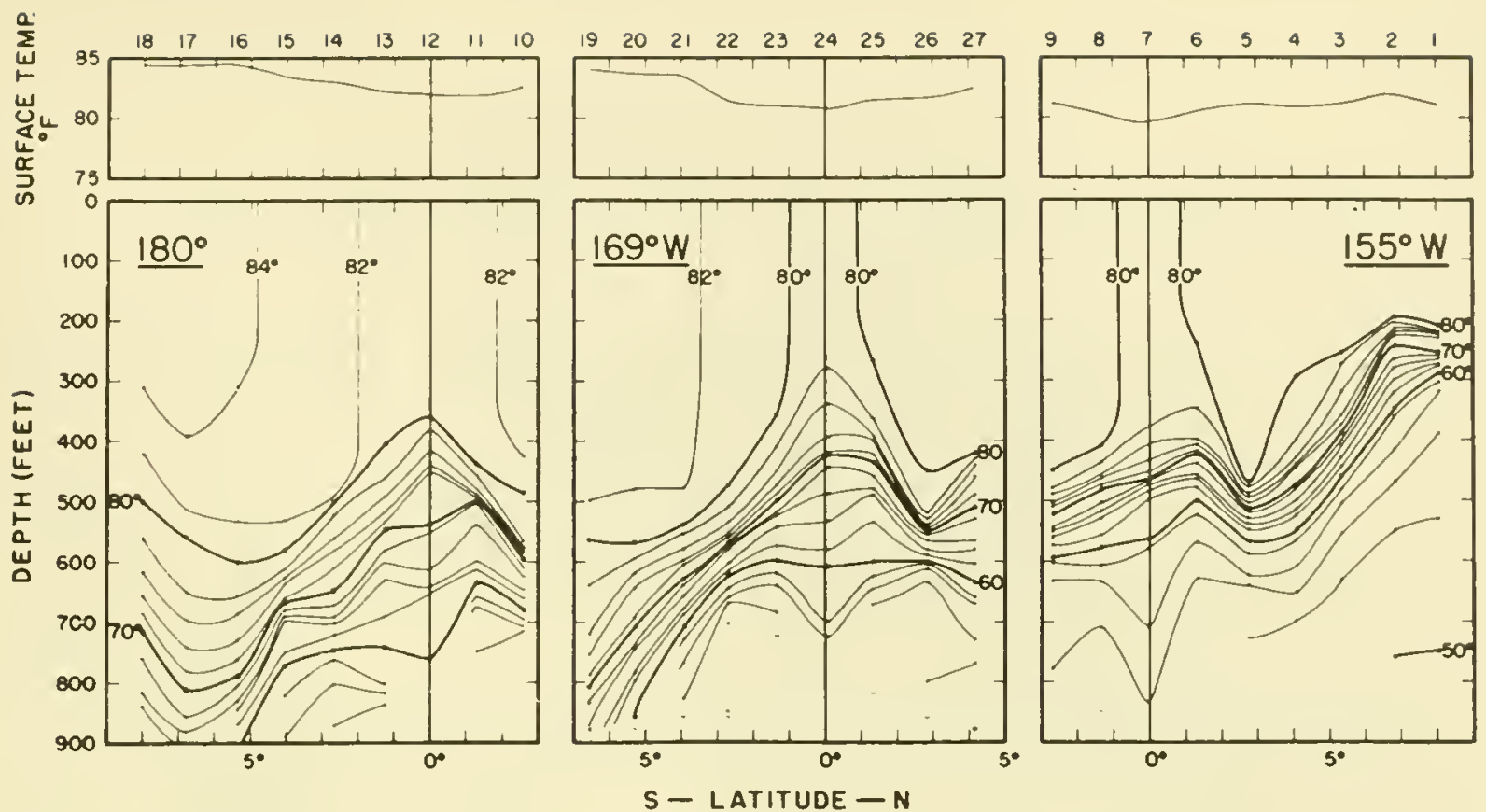

Fig. 16--Upper: surface temperatures by bucket thermometer in degrees F., measured with each BT cast. Lower: temperatures from BT data in degrees $\mathrm{F}$. John R. Manning cruise 1 l. Right: stations I (January 29) - 9, $155^{\circ} \mathrm{W}$. longitude, $8 \mathrm{O}^{\mathrm{N}}$. to $\overline{2^{\circ}} 40^{\prime} \mathrm{S} .1$ latitude, January-February 1952. Center: stations 19 (March 4) $-27,169^{\circ} \mathrm{W}$. longitude, $6^{\circ} 40^{\prime} \mathrm{S}$. to $4^{\circ} 10^{\prime} \mathrm{N}$. latitude, March 1952. Left: stations 10 (February 16) -18 , $180^{\circ}$ longitude, $2^{\circ} 35^{\prime} \mathrm{N}$. to $8^{\circ} \mathrm{S}$. latitude, February 1952 . Plotting interval $2^{\circ} \mathrm{F}$. Horizontal dashes indicate depths of observations less than 900 feet.

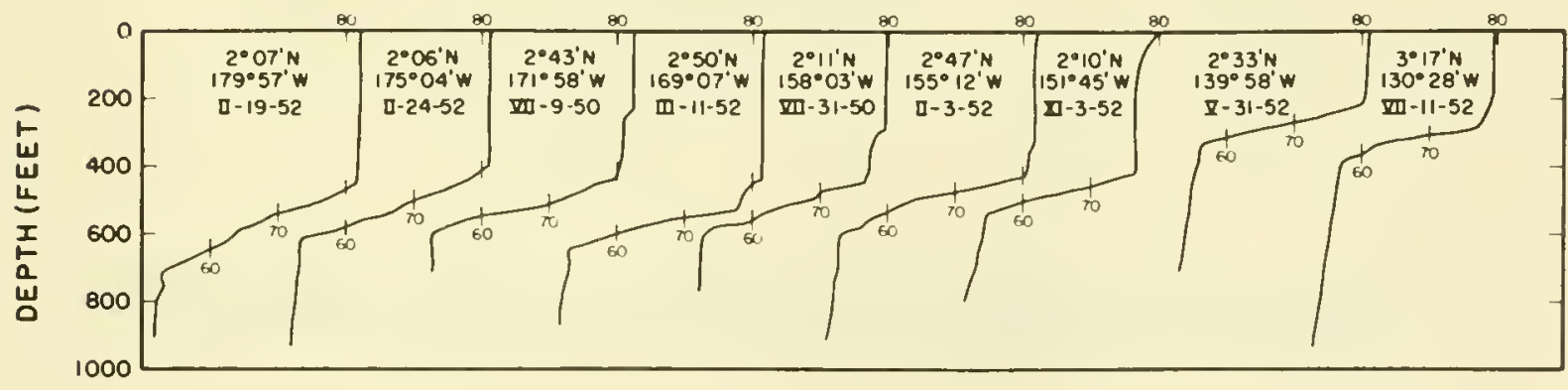

Fig. 17--BT traces illustrating the "sharp" thermocline bottom, $2^{\circ}-3^{\circ} \mathrm{N}$. latitude. Temperatures in degrees F., depths in feet. Position and date of each trace indicated. 


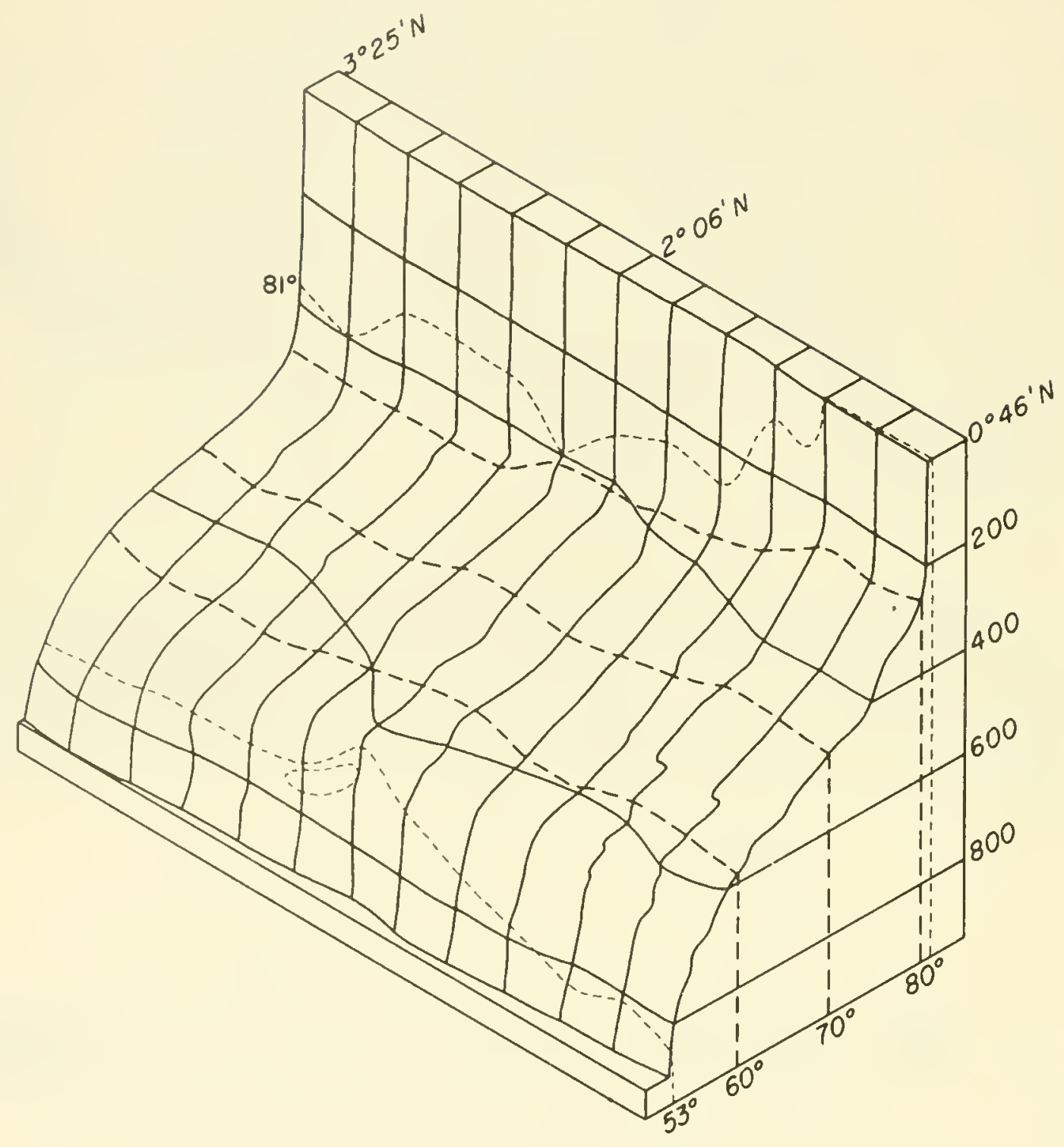

Fig. 18--Isometric block diagram presenting BT traces, $3^{\circ} 25^{\prime} \mathrm{N}$. latitude, $175^{\circ} 57 \mathrm{~W}$. longitude to $0^{\circ} 46^{\prime} \mathrm{N}$. latitude, $174^{\circ} 05^{\prime} \mathrm{W}$. Iongitude, Hugh. M. Smith cruise 14 .

Depth in feet, temperature in degrees $F$. 


\section{NOTES ON THE TABULATED DATA}

in a footnote.

In every case, any variation from the standard 13-bottle cast has been explained

Where more than one cast was made on a station, they are divided in the observed data by a horizontal line. The late $r$ casts are the deeper ones except on station 34 , where a single bottle was recast.

Where the corrected paired protected thermometer readings differed by more than $0.05^{\circ} \mathrm{C} .$, the depth and salinity are repeated, and both temperature values are carried. Sigma-t values calculated using each temperature are tabulated.

Where the duplicate determinations of inorganic phosphate differ in extinction value by more than 0.01 (approximately $0.05 \mu \mathrm{g}$ at $/ 1$ ), the depth is repeated, and both phosphate values are carried.

Weather is recorded in the ww (present weather) code given in the U. S. Weather Bureau Circular M, eighth edition, Manual of Marine Meteorological Observations. Cloud coverage is given in tenths of sky.

Wind velocity was measured with an anemometer 30 meters above the sea surface. The direction (given to the nearest $10^{\circ}$ ) is that from which the wind was blowing, measured clockwise through $360^{\circ}$ from north.

No phosphate determinations were made from station 6 to station 16 , and no data interpolated at standard depths for dynamic computations after station 19 (see text). 
STATION 1

M/V Hugh M. Smith: Cruise 14, $7^{\circ} 57 \cdot N, 154^{\circ} 57^{\prime} W$, January 27 , 1952. Messenger time: 2103 GCT. Weather: 03, cloud coverage 7. Wind: $070^{\circ}, 24 \mathrm{kt}$. Sea: $5-8 \mathrm{ft}$. Wire angle: $12^{\circ}$

O BSER VED

\begin{tabular}{|c|c|c|c|c|c|}
\hline $\begin{array}{c}\text { DEPTH } \\
\text { (m) }\end{array}$ & $\begin{array}{c}\mathrm{T} \\
\left({ }^{\circ} \mathrm{C}\right)\end{array}$ & $\begin{array}{c}5 \\
(0 / \infty)\end{array}$ & $\begin{array}{c}\sigma_{t} \\
(g / 1)\end{array}$ & $\begin{array}{c}\mathrm{O}_{2} \\
(\mathrm{ml} / \mathrm{l})\end{array}$ & $\begin{array}{c}\mathrm{PO}_{4}-\mathrm{P} \\
(\mu \mathrm{g} \text { at } / 1)\end{array}$ \\
\hline 00 & 27.43 & 34.18 & $21.9 \Xi$ & & 0.39 \\
\hline 11 & 27.44 & 34.18 & 21.98 & & 0.45 \\
\hline 26 & 27.40 & 34.20 & 22.01 & & 0.45 \\
\hline 55 & $27 \cdot 17$ & 34.29 & 22.15 & & 0.61 \\
\hline 109 & 13.35 & 34.44 & $25 \cdot 91$ & & $2 \cdot 31$ \\
\hline 215 & $10 \cdot 25$ & 34.66 & 26.66 & & $2 \cdot 34$ \\
\hline 321 & $09 \cdot 16$ & 34.63 & $26 \cdot 32$ & & 2.48 \\
\hline 432 & 08.39 & 34.56 & 26.89 & & 2.63 \\
\hline 538 & 07.35 & 34.53 & 27.02 & & 2.85 \\
\hline 646 & $26 \cdot 45$ & 34.47 & 27.10 & & 3.02 \\
\hline 853 & 05.04 & 34.52 & $27 \cdot 31$ & & 3.24 \\
\hline $\begin{array}{l}1064 \\
1266\end{array}$ & $\begin{array}{l}04.14 \\
03.54\end{array}$ & $34 \cdot 51$ & $27 \cdot 40$ & & 3.05 \\
\hline 1206 & 03.54 & 34.54 & 27.49 & & 2.93 \\
\hline
\end{tabular}

INTERPOLATED AND CALCULATED

\begin{tabular}{|c|c|c|c|c|c|}
\hline $\begin{array}{c}\text { DEPTH } \\
(\mathrm{m})\end{array}$ & $\begin{array}{c}\mathrm{T} \\
\left({ }^{\circ} \mathrm{C}\right)\end{array}$ & $\begin{array}{c}\mathrm{S} \\
(\% / 00)\end{array}$ & $\begin{array}{c}\sigma_{t} \\
(\mathrm{gm} / \mathrm{l})\end{array}$ & $\begin{array}{c}\Delta \mathrm{D} \\
(\mathrm{dyn} . \mathrm{m})\end{array}$ & $\begin{array}{c}\Delta \mathrm{D} 1000-\Delta \mathrm{D} \\
(\mathrm{dyn} . \mathrm{m})\end{array}$ \\
\hline
\end{tabular}

$\begin{array}{rlllll}00 & 27.43 & 34.13 & 21.98 & 0.000 & 1.505 \\ 10 & 27.42 & 34.19 & 21.99 & 0.058 & 1.537 \\ 20 & 27.42 & 34.19 & 22.00 & 0.117 & 1.478 \\ 30 & 27.40 & 34.20 & 22.01 & 0.175 & 1.420 \\ 50 & 27.20 & 34.27 & 22.13 & 0.291 & 1.304 \\ 75 & 25.10 & 34.51 & 22.96 & 0.424 & 1.171 \\ 100 & 15.58 & 34.46 & 25.45 & 0.512 & 1.077 \\ 150 & 11.94 & 34.49 & 26.23 & 0.629 & 0.966 \\ 200 & 10.61 & 34.63 & 26.57 & 0.713 & 0.822 \\ 250 & 09.79 & 34.66 & 26.74 & 0.736 & 0.810 \\ 300 & 09.35 & 34.65 & 26.21 & 0.253 & 0.742 \\ 400 & 02.62 & 34.58 & 26.27 & 0.922 & 0.613 \\ 500 & 07.71 & 34.54 & 26.98 & 1.105 & 0.490 \\ 600 & 06.87 & 34.49 & 27.06 & 1.220 & 0.376 \\ 700 & 06.03 & 34.48 & 27.16 & 1.326 & 0.269 \\ 800 & 05.33 & 34.51 & 27.27 & 1.423 & 0.173 \\ 1000 & 04.41 & 34.52 & 27.32 & 1.595 & 0.000\end{array}$




\section{STATION 2}

M/V Hugh M. Smith: Cruise $14,6^{\circ} 55^{\prime} N, 154^{\circ} 57^{\prime} W$, January 28 , 1952. Messenger time: $0647 \mathrm{GCT}$. Weather: 80 , cloud coverage 10. Wind: $030^{\circ}, 22 \mathrm{kt}$. Sea: 5-8 ft. Wire angle: $17^{\circ}$

OBSER VED

\begin{tabular}{|c|c|c|c|c|c|}
\hline $\begin{array}{c}\text { DEPTH } \\
\text { (m) }\end{array}$ & $\left({ }^{\mathrm{T}} \mathrm{C}\right)$ & $\begin{array}{c}S \\
(0 / 00)\end{array}$ & $\begin{array}{c}\sigma_{t} \\
(g / 1)\end{array}$ & $\begin{array}{c}\mathrm{O}_{2} \\
(\mathrm{ml} / \mathrm{l})\end{array}$ & $\begin{array}{l}\mathrm{PO}_{4}-\mathrm{P} \\
(\mu \mathrm{g} \text { at } / 1)\end{array}$ \\
\hline 00 & 27.93 & 34.65 & $22 \cdot 18$ & & 0.34 \\
\hline 11 & 27.96 & 34.60 & $22 \cdot 13$ & & 0.32 \\
\hline 11 & & & & & 0.32 \\
\hline 25 & $27 \cdot 94$ & 34.60 & $22 \cdot 13$ & & 0.36 \\
\hline 53 & $27 \cdot 96$ & 34.59 & $22 \cdot 12$ & & 0.47 \\
\hline 105 & 20.08 & 34.74 & 24.56 & & 0.72 \\
\hline 209 & 10.24 & 34.55 & 26.58 & & $2 \cdot 32$ \\
\hline 313 & 09.18 & 34.62 & $26 \cdot 81$ & & 2.56 \\
\hline $\begin{array}{l}423 \\
423\end{array}$ & $06 \cdot 34$ & 34.57 & 26.91 & & $\begin{array}{l}2.79 \\
2.86\end{array}$ \\
\hline 530 & 07.63 & 34.50 & 26.96 & & 2.97 \\
\hline 637 & 06.79 & 34.52 & 27.09 & & 3.08 \\
\hline $\begin{array}{c}1052 \\
1 /\end{array}$ & $04 \cdot 38$ & 34.50 & $27 \cdot 37$ & & $3 \cdot 24$ \\
\hline
\end{tabular}

INTERPOLATED AND CALCULATED

\begin{tabular}{|c|c|c|c|c|c|}
\hline $\begin{array}{l}\text { DEPTH } \\
\text { (m) }\end{array}$ & $\left({ }^{\circ} \mathrm{C}\right)$ & $\begin{array}{c}5 \\
(\% 00)\end{array}$ & $\begin{array}{c}\sigma_{\mathrm{t}} \\
(\mathrm{g} \mathrm{m} / 1)\end{array}$ & $\begin{array}{c}\Delta D \\
(d y n, m)\end{array}$ & $\begin{array}{c}\Delta D 1000-\Delta D \\
\text { (dyn. m) }\end{array}$ \\
\hline 00 & 27.93 & 34.65 & $22 \cdot 18$ & 0.000 & 1.663 \\
\hline 10 & 27.95 & 34.60 & 22.13 & 0.057 & 1.606 \\
\hline 20 & 27.95 & 34.60 & $22 \cdot 13$ & 0.114 & 1.549 \\
\hline 30 & 27.95 & 34.60 & 22.13 & 0.171 & 1.492 \\
\hline 50 & 27.96 & 34.59 & 22.12 & 0.286 & 1.378 \\
\hline 75 & 27.61 & 34.68 & $22 \cdot 30$ & 0.427 & $1 \cdot 236$ \\
\hline 100 & 21.00 & 34.77 & $24 \cdot 34$ & 0.542 & 1.121 \\
\hline 150 & 12.70 & 34.53 & $26 \cdot 11$ & 0.683 & 0.981 \\
\hline 200 & $10 \cdot 38$ & 34.55 & 26.55 & 0.770 & 0.893 \\
\hline 250 & 09.78 & 34.58 & 26.68 & 0.844 & 0.819 \\
\hline 300 & 09.29 & 34.61 & 26.79 & 0.913 & 0.750 \\
\hline 400 & 08.50 & 34.59 & 26.89 & 1.043 & 0.620 \\
\hline 500 & 07.89 & $34 \cdot 52$ & $26 \cdot 93$ & 1.166 & 0.497 \\
\hline 600 & 07.06 & 34.51 & 27.05 & 1.283 & 0.380 \\
\hline 700 & 06.24 & 34.52 & 27.16 & 1.390 & 0.273 \\
\hline 800 & 05.50 & 34.53 & $27 \cdot 27$ & 1.487 & 0.176 \\
\hline 1000 & 04.48 & 34.50 & 27.36 & 1.663 & 0.000 \\
\hline
\end{tabular}

1/ Two bottles pretripped, but were not recast. 
STATION 3

$\mathrm{M} / \mathrm{V}$ Hugh $\mathrm{M}$. Smith: Cruise $14,5^{\circ} 531 \mathrm{~N}, 154^{\circ} 55^{\prime} \mathrm{W}$, January 28 , 1952. Messenger time: $1708 \mathrm{GCT}$. Weather: 02 , cloud coverage 1. Wind: $040^{\circ}, 22 \mathrm{kt}$. Sea: $8-12 \mathrm{ft}$. Wire angle: $19^{\circ}$

OBSER VED

\begin{tabular}{|c|c|c|c|c|c|}
\hline $\begin{array}{c}\text { DEPTH } \\
\text { (m) }\end{array}$ & $\begin{array}{c}\mathrm{T} \\
\left({ }^{\circ} \mathrm{C}\right)\end{array}$ & $\begin{array}{c}S \\
(0 / \infty)\end{array}$ & $\begin{array}{l}\sigma_{t} \\
(g / 1)\end{array}$ & $\begin{array}{c}\mathrm{O}_{2} \\
(\mathrm{ml} / \mathrm{l})\end{array}$ & $\begin{array}{c}\mathrm{PO}_{4}-\mathrm{P} \\
(\mu 8 \text { at } / 1)\end{array}$ \\
\hline 01 & 27.84 & 34.65 & $22 \cdot 20$ & & 0.40 \\
\hline $\begin{array}{l}09 \\
09\end{array}$ & 27.87 & 34.66 & $22 \cdot 20$ & & $\begin{array}{l}0.45 \\
0.37\end{array}$ \\
\hline 24 & 27.82 & 34.63 & 22.20 & & 0.39 \\
\hline 53 & 27.73 & 34.74 & $22 \cdot 31$ & & 0.40 \\
\hline 105 & 26.16 & 35.09 & 23.07 & & 0.70 \\
\hline 209 & 10.66 & 34.62 & 26.56 & & 2.43 \\
\hline 313 & 09.16 & 34.64 & 26.83 & & $2 \cdot 49$ \\
\hline 423 & 02.45 & 34.61 & 26.92 & & 2.66 \\
\hline 527 & 07.65 & 34.55 & 26.99 & & 2.94 \\
\hline 632 & 06.85 & 34.56 & $27 \cdot 11$ & & 2.98 \\
\hline 835 & 05.92 & 34.52 & 27.20 & & 3.10 \\
\hline 1039 & 04.40 & 34.54 & 27.40 & & 3.18 \\
\hline 1237 & 03.74 & 34.56 & 27.48 & & 3.07 \\
\hline 1237 & & & & & $3 \cdot 21$ \\
\hline
\end{tabular}

INTERPOLATED AND CALCULATED

\begin{tabular}{|c|c|c|c|c|c|}
\hline $\begin{array}{c}\text { DEPTH } \\
(\mathrm{m})\end{array}$ & $\begin{array}{c}\mathrm{T} \\
\left({ }^{\circ} \mathrm{C}\right)\end{array}$ & $\begin{array}{c}\mathrm{S} \\
(\% / \mathrm{oo})\end{array}$ & $\begin{array}{c}\sigma_{\mathrm{t}} \\
(\mathrm{gm} / \mathrm{m})\end{array}$ & $\begin{array}{c}\Delta \mathrm{D} \\
(\mathrm{dyn}, \mathrm{m})\end{array}$ & $\begin{array}{c}\Delta \mathrm{D} 1000-\Delta D \\
(\mathrm{dyn} . \mathrm{m})\end{array}$ \\
\hline
\end{tabular}

$\begin{array}{rlllll}00 & 27.84 & 34.65 & 22.20 & 0.000 & 1.744 \\ 10 & 27.87 & 34.66 & 22.20 & 0.056 & 1.688 \\ 20 & 27.84 & 34.64 & 22.20 & 0.113 & 1.631 \\ 30 & 27.80 & 34.65 & 22.22 & 0.169 & 1.575 \\ 50 & 27.76 & 34.73 & 22.29 & 0.281 & 1.463 \\ 75 & 27.22 & 34.95 & 22.63 & 0.417 & 1.327 \\ 100 & 26.40 & 35.08 & 22.99 & 0.544 & 1.200 \\ 150 & 17.00 & 34.73 & 25.32 & 0.736 & 1.008 \\ 200 & 11.00 & 34.62 & 26.50 & 0.844 & 0.900 \\ 250 & 10.01 & 34.63 & 26.62 & 0.919 & 0.825 \\ 300 & 09.30 & 34.64 & 26.31 & 0.928 & 0.756 \\ 400 & 08.58 & 34.62 & 26.91 & 1.116 & 0.623 \\ 500 & 07.92 & 34.57 & 26.97 & 1.237 & 0.507 \\ 600 & 07.05 & 34.56 & 27.09 & 1.351 & 0.393 \\ 700 & 06.60 & 34.55 & 27.14 & 1.457 & 0.287 \\ 800 & 06.15 & 34.53 & 27.19 & 1.560 & 0.124 \\ 1000 & 04.67 & 34.54 & 27.37 & 1.744 & 0.000\end{array}$




\section{STATION 4}

M/V Hugh M. Smith: Cruise $14,4^{\circ} 55^{\prime} N, 154^{\circ} 51^{\prime} \mathrm{W}$, January 29. 1952. Messenger time: $0227 \mathrm{GCT}$. Weather: 02, cloud coverage 1. Wind: $060^{\circ}, 22 \mathrm{kt}$. Sea: $8-12 \mathrm{ft}$. Wire angle: $15^{\circ}$

O BSER VE D

\begin{tabular}{c|cc|c|c|c|}
\hline $\begin{array}{c}\text { DEPTH } \\
(\mathrm{m})\end{array}$ & $\begin{array}{c}\mathrm{T} \\
\left({ }^{\circ} \mathrm{C}\right)\end{array}$ & $\begin{array}{c}\mathrm{S} \\
(\% / 00)\end{array}$ & $\begin{array}{c}\sigma_{\mathrm{t}} \\
(\mathrm{g} / 1)\end{array}$ & $\begin{array}{c}\mathrm{O}_{2} \\
(\mathrm{ml} / \mathrm{l})\end{array}$ & $\begin{array}{c}\mathrm{PO}_{4}^{-\mathrm{P}} \\
(\mu \mathrm{g} \text { at } / 1)\end{array}$ \\
\hline 00 & 27.25 & 35.08 & 22.72 & & 0.77 \\
10 & 27.26 & 35.07 & 22.71 & & 0.71 \\
10 & & & & 0.63 \\
24 & 27.17 & 35.04 & 22.71 & & 0.79 \\
24 & & & & 0.64 \\
52 & 27.02 & 35.04 & 22.76 & & 0.60 \\
105 & 26.71 & 35.16 & 22.95 & & 0.79 \\
$2071 /$ & 10.84 & & & \\
309 & 09.26 & 34.65 & 26.37 & & 2.29 \\
309 & & & & 2.52 \\
416 & 08.70 & 34.60 & 26.87 & & 2.71 \\
518 & 07.99 & 34.58 & 26.97 & & 3.91 \\
620 & 07.14 & 34.57 & 27.08 & & 2.84 \\
82121 & 05.79 & 34.52 & 27.22 & & 3.14 \\
821 & 05.82 & 34.52 & 27.22 & & 2.27 \\
1027 & 04.60 & 34.54 & 27.38 & & 2.51 \\
1027 & 04.60 & 34.54 & 27.38 & & 2.20 \\
1225 & 03.84 & 34.55 & 27.46 & & \\
1225 & & & & &
\end{tabular}

INTERPOLATED AND CALCULATED

\begin{tabular}{|c|c|c|c|c|c|}
\hline $\begin{array}{c}\text { DEPTH } \\
(\mathrm{m})\end{array}$ & $\begin{array}{c}\mathrm{T} \\
\left({ }^{\circ} \mathrm{C}\right)\end{array}$ & $\begin{array}{c}\mathrm{S} \\
(\% / \mathrm{oo})\end{array}$ & $\begin{array}{c}\sigma_{\mathrm{t}} \\
(\mathrm{gm} / \mathrm{l})\end{array}$ & $\begin{array}{c}\Delta \mathrm{D} \\
(\mathrm{dyn} . \mathrm{m})\end{array}$ & $\begin{array}{c}\Delta \mathrm{D} 1000-\Delta \mathrm{D} \\
(\mathrm{dyn} . \mathrm{m})\end{array}$ \\
\hline 00 & 27.25 & 35.08 & 22.72 & 0.000 & 1.775 \\
10 & 27.26 & 35.07 & 22.71 & 0.051 & 1.723 \\
20 & 27.20 & 35.04 & 22.71 & 0.103 & 1.672 \\
30 & 27.10 & 35.04 & 22.74 & 0.155 & 1.620 \\
50 & 27.02 & 35.04 & 22.76 & 0.257 & 1.518 \\
75 & 26.89 & 35.14 & 22.82 & 0.384 & 1.391 \\
100 & 26.77 & 35.16 & 22.94 & 0.509 & 1.266 \\
150 & 22.00 & 34.94 & 24.19 & 0.729 & 1.046 \\
200 & 11.90 & 34.62 & 26.33 & 0.869 & 0.906 \\
250 & 09.64 & 34.65 & 26.76 & 0.946 & 0.829 \\
300 & 09.30 & 34.65 & 26.81 & 1.013 & 0.762 \\
400 & 08.79 & 34.61 & 26.87 & 1.142 & 0.633 \\
500 & 08.10 & 34.52 & 26.95 & 1.267 & 0.508 \\
600 & 07.28 & 34.57 & 27.06 & 1.383 & 0.392 \\
700 & 06.57 & 34.54 & 27.14 & 1.491 & 0.284 \\
800 & 05.90 & 34.52 & 27.21 & 1.592 & 0.183 \\
1000 & 04.74 & 34.53 & 27.35 & 1.775 & 0.000
\end{tabular}

1/ Phosphate and salinity values are not good; temperature seems all right.

2/ Both the temperature and phosphate duplicates were out of tolerance for this observation. 


\section{STATION 5}

$\mathrm{M} / \mathrm{V}$ Hugh $\mathrm{M}$. Smith: Cruise $14,3^{\circ} 54^{\prime} \mathrm{N}, 154^{\circ} 51^{\prime} \mathrm{W}$, January 29, 1952. Messenger time: 1128 GCT. Weather: 02, cloud coverage 1 . Wind: $040^{\circ}, 15 \mathrm{kt}$. Sea: $5-8 \mathrm{ft}$. Wire angle: $38^{\circ}$

OBSER VED

\begin{tabular}{|c|c|c|c|c|c|}
\hline $\begin{array}{c}\text { DEPTH } \\
\text { (m) }\end{array}$ & $\left(\begin{array}{c}\mathrm{T} \\
\left({ }^{\circ} \mathrm{C}\right)\end{array}\right.$ & $\begin{array}{c}S \\
(\% / 00)\end{array}$ & $\begin{array}{c}\sigma_{t} \\
(\mathrm{~g} / 1)\end{array}$ & $\begin{array}{c}\mathrm{O}_{2} \\
(\mathrm{ml} / \mathrm{l})\end{array}$ & $\begin{array}{c}\mathrm{PO}_{4}-\mathrm{P} \\
(\mu \mathrm{g} \text { at } / 1)\end{array}$ \\
\hline $\begin{array}{l}00 \\
08 \\
20 \\
44 \\
84 \\
159 \\
230 \\
305 \\
376 \\
447 \\
592 \\
592 \\
755 \\
925\end{array}$ & $\begin{array}{l}27.17 \\
27.20 \\
27.12 \\
27.19 \\
27.07 \\
23.61 \\
10.80 \\
09.16 \\
08.75 \\
08.26 \\
06.88 \\
06.93 \\
05.77 \\
04.88\end{array}$ & $\begin{array}{l}35.14 \\
35.17 \\
35.14 \\
35.14 \\
35.12 \\
34.92 \\
34.61 \\
34.62 \\
34.61 \\
34.60 \\
34.52 \\
34.52 \\
34.52 \\
34.52\end{array}$ & $\begin{array}{l}22 \cdot 79 \\
22 \cdot 80 \\
22 \cdot 79 \\
22.78 \\
22.80 \\
23.76 \\
26.53 \\
26.82 \\
26.87 \\
26.94 \\
27.08 \\
27.07 \\
27.22 \\
27.33\end{array}$ & & $\begin{array}{l}0.72 \\
0.73 \\
0.75 \\
0.69 \\
0.35 \\
0.44 \\
2.02 \\
2.16 \\
2.26 \\
2.50 \\
2.83\end{array}$ \\
\hline
\end{tabular}

INTERPOLATED AND CALCULATED

\begin{tabular}{|c|c|c|c|c|c|}
\hline $\begin{array}{c}\text { DEPTH } \\
(\mathrm{m})\end{array}$ & $\begin{array}{c}\mathrm{T} \\
\left({ }^{\circ} \mathrm{C}\right)\end{array}$ & $\begin{array}{c}\mathrm{s} \\
(\%)\end{array}$ & $\begin{array}{c}\sigma_{\mathrm{t}} \\
(\mathrm{gm} / \mathrm{l})\end{array}$ & $\begin{array}{c}\Delta \mathrm{D} \\
(\mathrm{dyn}, \mathrm{m})\end{array}$ & $\begin{array}{c}\Delta \mathrm{D} 1000-\Delta \mathrm{D} \\
(\mathrm{dyn}, \mathrm{m})\end{array}$ \\
\hline
\end{tabular}

$\begin{array}{rlllll}00 & 27.17 & 35.14 & 22.79 & 0.000 & 1.801 \\ 10 & 27.19 & 35.16 & 22.80 & 0.051 & 1.751 \\ 20 & 27.18 & 35.14 & 22.79 & 0.102 & 1.700 \\ 30 & 27.18 & 35.14 & 22.79 & 0.152 & 1.649 \\ 50 & 27.16 & 35.14 & 22.79 & 0.254 & 1.547 \\ 75 & 27.10 & 35.12 & 22.80 & 0.382 & 1.420 \\ 100 & 26.98 & 35.11 & 22.83 & 0.509 & 1.292 \\ 150 & 24.10 & 35.01 & 23.64 & 0.745 & 1.056 \\ 200 & 13.71 & 34.58 & 25.94 & 0.907 & 0.894 \\ 250 & 10.00 & 34.63 & 26.68 & 0.996 & 0.805 \\ 300 & 09.17 & 34.62 & 26.81 & 1.064 & 0.737 \\ 400 & 08.57 & 34.61 & 26.90 & 1.192 & 0.609 \\ 500 & 07.68 & 34.56 & 27.00 & 1.312 & 0.489 \\ 600 & 06.85 & 34.52 & 27.08 & 1.425 & 0.376 \\ 700 & 06.13 & 34.52 & 27.18 & 1.529 & 0.272 \\ 800 & 05.45 & 34.52 & 27.26 & 1.625 & 0.176 \\ 100011 & 04.62 & 34.52 & 27.36 & 1.301 & 0.000\end{array}$

1/ Extrapolated values. 
M/V Hugh M. Smith: Cruise $14,2^{\circ} 56{ }^{\prime} \mathrm{N}, 154^{\circ} 59^{\prime} \mathrm{W}$, January 29. 1952. Messenger time: $2023 \mathrm{GCT}$. Weather: 02, cloud coverage 4 . Wind: $040^{\circ}, 19 \mathrm{kt}$. Sea: $5-8 \mathrm{ft}$. Wire angle: $47^{\circ}$

OBSER VED

\begin{tabular}{|c|c|c|c|c|c|}
\hline $\begin{array}{c}\text { DEPTH } \\
(\mathrm{m})\end{array}$ & $\begin{array}{c}\mathrm{T} \\
\left({ }^{\circ} \mathrm{C}\right)\end{array}$ & $\begin{array}{c}\mathrm{S} \\
\left({ }^{\circ} / \mathrm{oo}\right)\end{array}$ & $\begin{array}{c}\sigma_{\mathrm{t}} \\
(\mathrm{g} / 1)\end{array}$ & $\begin{array}{c}\mathrm{O}_{2} \\
(\mathrm{ml} / 1)\end{array}$ & $\begin{array}{c}\mathrm{PO}_{4}-\mathrm{P} \\
(\mu \mathrm{g} \text { at } / 1)\end{array}$ \\
\hline 00 & 27.10 & 35.08 & 22.77 & \\
08 & 27.12 & 35.07 & 22.75 & \\
20 & 27.07 & 35.08 & 22.77 & \\
41 & 27.07 & 35.08 & 22.77 & \\
77 & 27.07 & 35.07 & 22.77 & \\
141 & 26.76 & 35.11 & 22.90 & \\
190 & 12.59 & 34.58 & 26.17 & \\
240 & 10.39 & 34.69 & 26.66 & \\
295 & 10.06 & 34.69 & 26.72 & \\
350 & 09.43 & 34.67 & 26.31 & \\
475 & 08.53 & 34.61 & 26.91 & \\
475 & 08.60 & 34.61 & 26.89 & \\
607 & 06.86 & 34.54 & 27.10 & \\
758 & 05.62 & 34.51 & 27.23 &
\end{tabular}

INTERPOLATED AND CALCULATED

\begin{tabular}{|c|c|c|c|c|c|}
\hline $\begin{array}{c}\text { DEPTH } \\
\text { (m) }\end{array}$ & $\left({ }^{\circ} \mathrm{C}\right)$ & $\begin{array}{c}5 \\
(0 / 00)\end{array}$ & $\begin{array}{c}\sigma_{\mathrm{t}} \\
(\mathrm{gm} / 1)\end{array}$ & $\begin{array}{c}\Delta D \\
(\mathrm{dyn}, \mathrm{m})\end{array}$ & $\begin{array}{c}\Delta D 1000-\triangle D \\
\text { (dyn. m) }\end{array}$ \\
\hline $\begin{array}{l}60 \\
10 \\
20 \\
30 \\
50 \\
75 \\
100 \\
150 \\
200 \\
250 \\
300 \\
400 \\
500 \\
600 \\
700 \\
80011 \\
1000 \mathrm{II}\end{array}$ & $\begin{array}{l}27.10 \\
27.10 \\
27.07 \\
27.07 \\
27.07 \\
27.07 \\
26.98 \\
20.10 \\
11.62 \\
10.28 \\
09.97 \\
09.18 \\
09.14 \\
06.96 \\
66.07 \\
05.40 \\
04.08\end{array}$ & $\begin{array}{l}35.05 \\
35.07 \\
35.08 \\
35.05 \\
35.07 \\
35.07 \\
35.09 \\
34.83 \\
34.62 \\
34.69 \\
34.69 \\
34.66 \\
34.58 \\
34.54 \\
34.52 \\
34.52 \\
34.56\end{array}$ & $\begin{array}{l}22 \cdot 77 \\
22.76 \\
22 \cdot 73 \\
22 \cdot 78 \\
22.77 \\
22.77 \\
22.32 \\
24.63 \\
26.39 \\
26.68 \\
26.73 \\
26.84 \\
26.94 \\
27.08 \\
27.19 \\
27.27 \\
27.36\end{array}$ & $\begin{array}{l}0.000 \\
0.051 \\
0.102 \\
0.153 \\
0.255 \\
0.583 \\
0.511 \\
0.724 \\
0.851 \\
0.930 \\
1.000 \\
1.135 \\
1.260 \\
1.376 \\
1.480 \\
1.575 \\
1.750\end{array}$ & $\begin{array}{l}1.750 \\
1.699 \\
1.648 \\
1.597 \\
1.495 \\
1.367 \\
1.239 \\
1.026 \\
0.899 \\
0.820 \\
0.750 \\
0.615 \\
0.429 \\
0.374 \\
0.270 \\
0.175 \\
0.000\end{array}$ \\
\hline
\end{tabular}




\section{STATION 7}

M/V Hugh M. Smith: Cruise $14, I^{\circ} 54 ' N, 155^{\circ} 03^{\prime} \mathrm{W}$, January 30 , 1952. Messenger time: First cast $0655 \mathrm{GCT}$, second cast 0729 GCT. Weather: 50 , cloud coverage 10 . Wind: $050^{\circ}$, $18 \mathrm{kt}$. Sea: 5-8 ft. Wire angle:- First cast $58^{\circ}$, second cast $40^{\circ}$

OBSER VED

\begin{tabular}{|c|c|c|c|c|c|}
\hline $\begin{array}{c}\text { DEPTH } \\
(\mathrm{m})\end{array}$ & $\begin{array}{c}\mathrm{T} \\
\left({ }^{\circ} \mathrm{C}\right)\end{array}$ & $\begin{array}{c}\mathrm{S} \\
(\mathrm{o} / \mathrm{oo})\end{array}$ & $\begin{array}{c}\sigma_{\mathrm{t}} \\
(\mathrm{g} / 1)\end{array}$ & $\begin{array}{c}\mathrm{O}_{2} \\
(\mathrm{ml} / 1)\end{array}$ & $\begin{array}{c}\mathrm{PO}_{4}-\mathrm{P} \\
(\mu \mathrm{gt} / 1)\end{array}$ \\
\hline 00 & 27.40 & 35.11 & 22.71 & \\
13 & 27.42 & 35.10 & 22.68 & \\
29 & 27.38 & 35.08 & 22.68 & \\
59 & 27.32 & 35.11 & 22.72 \\
\hline 117 & 25.00 & 35.07 & 23.41 & \\
231 & 11.40 & 34.80 & 26.56 & \\
341 & 10.81 & 34.70 & 26.60 & \\
463 & 09.32 & 34.68 & 26.84 & \\
575 & 08.08 & 34.61 & 26.98 & \\
696 & 06.58 & 34.52 & 27.12 & \\
938 & 04.83 & 34.51 & 27.33 & \\
938 & 04.85 & 34.51 & 27.33 & \\
1209 & 03.74 & 34.54 & 27.47 & \\
21 & & & &
\end{tabular}

INTERPOLATED AND CALCULATED

\begin{tabular}{|c|c|c|c|c|c|}
\hline $\begin{array}{c}\text { DEPTH } \\
(\mathrm{m})\end{array}$ & $\begin{array}{c}\mathrm{T} \\
\left({ }^{\circ} \mathrm{C}\right)\end{array}$ & $\begin{array}{c}\mathrm{S} \\
\left({ }^{\circ} / \mathrm{oo}\right)\end{array}$ & $\begin{array}{c}\sigma_{\mathrm{t}} \\
(\mathrm{gm} / \mathrm{l})\end{array}$ & $\begin{array}{c}\Delta \mathrm{D} \\
(\mathrm{dyn} . \mathrm{m})\end{array}$ & $\begin{array}{c}\Delta \mathrm{D} 1000-\Delta \mathrm{D} \\
(\mathrm{d} y \mathbf{y n} . \mathrm{m})\end{array}$ \\
\hline 00 & 27.40 & 35.11 & 22.69 & 0.000 & 1.770 \\
10 & 27.41 & 35.11 & 22.69 & 0.052 & 1.718 \\
20 & 27.40 & 35.09 & 22.68 & 0.103 & 1.666 \\
30 & 27.38 & 35.08 & 22.68 & 0.155 & 1.614 \\
50 & 27.34 & 35.11 & 22.71 & 0.259 & 1.511 \\
75 & 27.30 & 35.11 & 22.73 & 0.388 & 1.381 \\
100 & 27.22 & 35.11 & 22.75 & 0.518 & 1.252 \\
150 & 16.62 & 34.80 & 25.47 & 0.711 & 1.058 \\
200 & 11.76 & 34.83 & 26.52 & 0.815 & 0.954 \\
250 & 11.37 & 34.80 & 26.57 & 0.893 & 0.876 \\
300 & 11.13 & 34.75 & 26.58 & 0.971 & 0.799 \\
400 & 10.10 & 34.68 & 26.70 & 1.120 & 0.650 \\
500 & 08.96 & 34.66 & 26.88 & 1.256 & 0.513 \\
600 & 07.71 & 34.58 & 27.01 & 1.379 & 0.391 \\
700 & 06.50 & 34.52 & 27.13 & 1.490 & 0.279 \\
800 & 05.54 & 34.51 & 27.23 & 1.591 & 0.179 \\
1000 & 04.58 & 34.52 & 27.36 & 1.770 & 0.000
\end{tabular}

$1 /$ Under way to reduce wire angle.

2/ Bottom bottle pretripped, but was not recast. 
M/V Hugh M. Smith: Cruise $14,0^{\circ} 54 ' N, 155^{\circ} 11 ' \mathrm{~W}$, January 30 , 1952. Messenger time: First cast $1638 \mathrm{GCT}$, second cast 1726 GCT, third cast $1811 \mathrm{GCT}$. Weather: 01 , cloud coverage 1. Wind: $020^{\circ}, 13 \mathrm{kt}$. Sea: $3-5 \mathrm{ft}$. Wire angle: First cast $50^{\circ}, 1 /$ second cast $55^{\circ}$

OBSER VED

\begin{tabular}{c|c|c|c|c|c|}
\hline $\begin{array}{c}\text { DEPTH } \\
(\mathrm{m})\end{array}$ & $\begin{array}{c}\mathrm{T} \\
\left({ }^{\circ} \mathrm{C}\right)\end{array}$ & $\begin{array}{c}\mathrm{S} \\
(\% / \mathrm{oo})\end{array}$ & $\begin{array}{c}\sigma_{\mathrm{t}} \\
(\mathrm{g} / \mathrm{l})\end{array}$ & $\begin{array}{c}\mathrm{O}_{2} \\
(\mathrm{ml} / \mathrm{l})\end{array}$ & $\begin{array}{c}\mathrm{PO}_{4}-\mathrm{P} \\
(\mu \mathrm{g} \text { at } / 1)\end{array}$ \\
\hline 00 & 27.25 & 35.06 & 22.70 & \\
10 & 27.26 & 35.03 & 22.68 & \\
21 & 27.24 & 35.04 & 22.69 \\
\hline 42 & 27.22 & 35.06 & 22.71 \\
74 & 27.04 & 35.06 & 22.77 & \\
139 & 19.00 & 34.82 & 24.90 & \\
202 & 12.72 & 34.88 & 26.37 & \\
267 & 11.66 & 34.84 & 25.54 & \\
332 & 10.43 & 34.74 & 26.69 \\
21 & &
\end{tabular}

INTERPOLATED AND CALCULATED

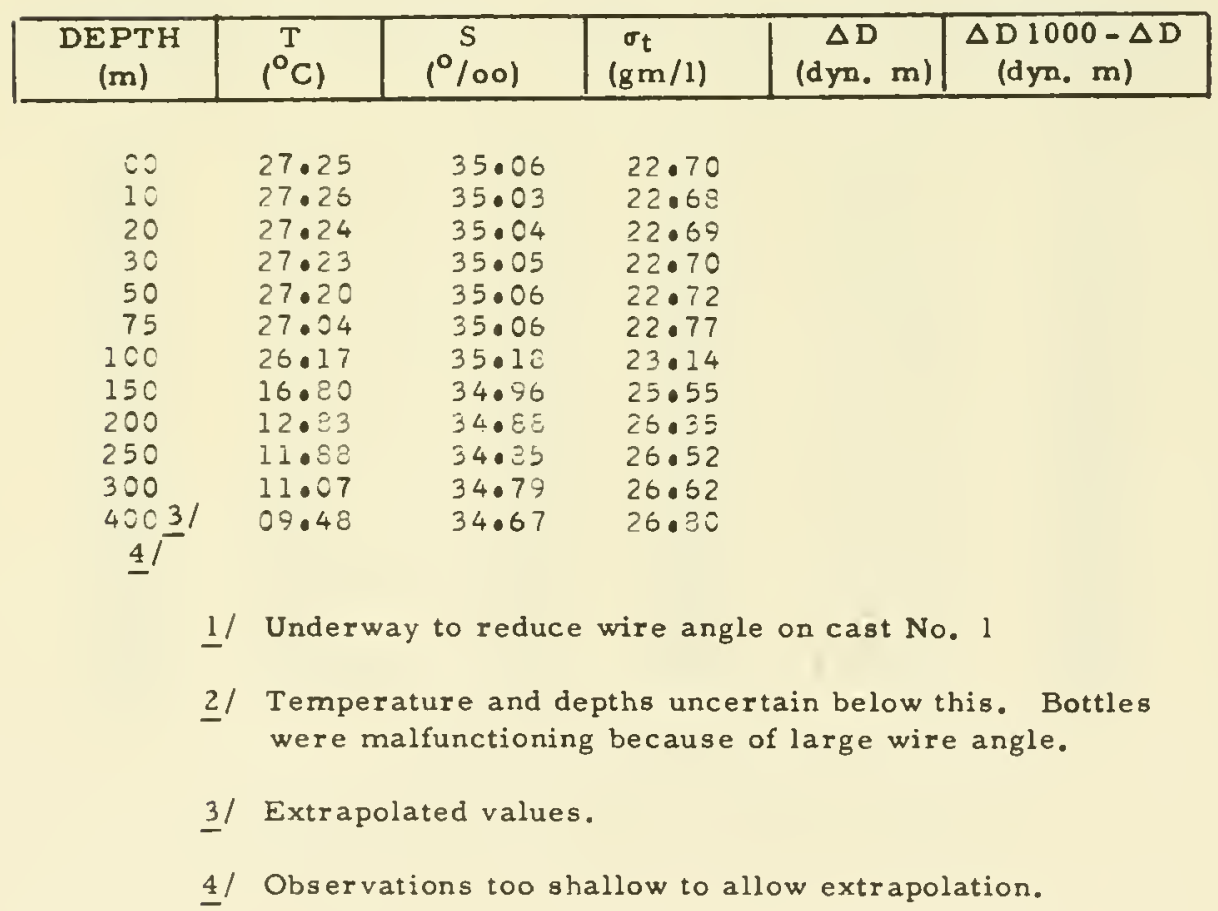




\section{STATION 9}

M/V Hugh M. Smith: Cruise $14,0^{\circ} 06 ' S, 155^{\circ} 14 ' \mathrm{~W}$, January 31 , 1952. Messenger time: 0421 GCT. Weather: 02, cloud coverage 4. Wind: $060^{\circ}, 8 \mathrm{kt}$. Sea: < I ft. Wire angle: $1 / 48^{\circ}$

OBSER VED

\begin{tabular}{|c|c|c|c|c|c|}
\hline $\begin{array}{c}\text { DEPTH } \\
(\mathrm{m})\end{array}$ & $\begin{array}{c}\mathrm{T} \\
\left({ }^{\circ} \mathrm{C}\right)\end{array}$ & $\begin{array}{c}\mathbf{S} \\
\left({ }^{\circ} / \mathrm{oo}\right)\end{array}$ & $\begin{array}{c}\sigma_{\mathrm{t}} \\
(\mathrm{g} / 1)\end{array}$ & $\begin{array}{c}\mathrm{O}_{2} \\
(\mathrm{ml} / 1)\end{array}$ & $\begin{array}{c}\mathrm{PO}_{4}-\mathbf{P} \\
(\mu \mathrm{g} / 1)\end{array}$ \\
\hline 02 & 27.31 & 35.04 & 22.57 & \\
14 & 27.14 & 35.04 & 22.72 & \\
30 & 26.96 & 35.07 & 22.80 & \\
60 & 26.68 & 35.23 & 23.01 & \\
116 & 25.35 & 35.26 & 23.45 & \\
222 & 13.04 & 34.97 & 26.38 & \\
324 & 11.46 & 34.82 & 26.57 & \\
437 & 09.30 & 34.67 & 26.83 & \\
543 & 07.51 & 34.60 & 27.05 & \\
659 & 06.31 & 34.54 & 27.17 & \\
894 & 04.94 & 34.55 & 27.35 & \\
894 & 04.89 & 34.55 & 27.36 & \\
1145 & 03.83 & 34.56 & 27.67 & \\
21 & & & &
\end{tabular}

INTERPOLATED AND CALCULATED

\begin{tabular}{|c|c|c|c|c|c|}
\hline $\begin{array}{c}\text { DEPTH } \\
\text { (m) }\end{array}$ & $\left({ }^{\circ} \mathrm{C}\right)$ & $\begin{array}{c}5 \\
(\% / 00)\end{array}$ & $\begin{array}{c}\sigma_{\mathrm{t}} \\
(\mathrm{gm} / \mathrm{m})\end{array}$ & $\begin{array}{c}\Delta D \\
(\mathrm{dyn}, \mathrm{m})\end{array}$ & $\begin{array}{r}\Delta \mathrm{D} 1000- \\
\text { (dyn. m) }\end{array}$ \\
\hline 00 & $27 \cdot 31$ & 35.04 & 22.67 & 0.000 & 1.720 \\
\hline 10 & 27.20 & 35.04 & 22.71 & 0.052 & 1.66 \\
\hline 20 & 27.05 & 35.05 & 22.76 & 0.103 & 1.61 \\
\hline 30 & 26.96 & 35.07 & 22.31 & 0.154 & 1.56 \\
\hline 50 & $26 \cdot 79$ & $35 \cdot 18$ & $22 \cdot 54$ & 0.254 & 1.466 \\
\hline 75 & 26.50 & $35 \cdot 25$ & 23.09 & 0.376 & $1 \cdot 34$ \\
\hline 100 & 25.89 & $35 \cdot 26$ & 23.28 & 0.495 & $1 \cdot 22$ \\
\hline 150 & 18.60 & 35.08 & 25.20 & 0.602 & 1.03 \\
\hline 200 & 13.43 & 35.00 & $26 \cdot 32$ & 0.798 & 0.92 \\
\hline 250 & 12.60 & 34.93 & $26 \cdot 44$ & 0.234 & 0.83 \\
\hline 300 & 11.83 & 34.86 & 26.53 & 0.966 & 0.75 \\
\hline 400 & 10.00 & 34.71 & $26 \cdot 74$ & 1.115 & 0.60 \\
\hline 500 & 08.20 & 34.62 & 26.97 & $1 \cdot 245$ & 0.47 \\
\hline 600 & 06.85 & 34.56 & 27.11 & $1 \cdot 358$ & 0.36 \\
\hline 700 & 06.00 & $34 \cdot 54$ & $27 \cdot 21$ & $1 \cdot 459$ & 0.26 \\
\hline 800 & 05.39 & 34.55 & $27 \cdot 30$ & 1.552 & 0.16 \\
\hline 1000 & $04 \cdot 43$ & 34.55 & 27.40 & 1.720 & 0.00 \\
\hline
\end{tabular}

1/ Underway to reduce wire angle.

2/ Bottom bottle pretripped, but was not recast. 


\section{STATION 10}

M/V Hugh M. Smith: Cruise 14, $1^{\circ} 10^{\prime} \mathrm{S}, 155^{\circ} 08^{\prime} \mathrm{W}, \mathrm{January} 31$, 1952. Messenger time: 1405 GCT. Weather: 02, cloud coverage 2. Wind: $030^{\circ}, 6 \mathrm{kt}$. Sea: < $1 \mathrm{ft}$. Wire angle: $25^{\circ}$

OBSER V E D

\begin{tabular}{c|cc|c|c|c|}
\hline $\begin{array}{c}\text { DEPTH } \\
(\mathrm{m})\end{array}$ & $\begin{array}{c}\mathrm{T} \\
\left({ }^{\circ} \mathrm{C}\right)\end{array}$ & $\begin{array}{c}\mathrm{S} \\
\left({ }^{\circ} / 00\right)\end{array}$ & $\begin{array}{c}\sigma_{t} \\
(\mathrm{~g} / 1)\end{array}$ & $\begin{array}{c}\mathrm{O}_{2} \\
(\mathrm{ml} / 1)\end{array}$ & $\begin{array}{c}\mathrm{PO}_{4}-\mathrm{P} \\
(\mu \mathrm{g} \text { at } / 1)\end{array}$ \\
\hline 00 & 26.87 & 35.31 & 23.02 & \\
14 & 26.72 & 35.29 & 23.05 & \\
32 & 26.64 & 35.31 & 23.09 & \\
66 & 26.56 & 35.32 & 23.12 & \\
129 & 25.55 & 35.47 & 23.55 & \\
252 & 11.72 & 34.95 & 26.54 & \\
371 & 10.50 & 34.79 & 26.67 & \\
498 & 07.94 & 34.61 & 77.00 & \\
620 & 06.69 & 34.54 & 27.12 & \\
749 & 05.93 & 34.52 & 27.20 & \\
1015 & 04.40 & 34.53 & 27.39 & \\
1015 & 04.45 & 34.53 & 27.39 & \\
1293 & 03.44 & 34.60 & 27.54 & \\
1585 & 03.02 & 34.59 & 27.52 &
\end{tabular}

INTERPOLATED AND CALCULATED

\begin{tabular}{|c|c|c|c|c|c|}
\hline $\begin{array}{l}\text { DEPTH } \\
\text { (m) }\end{array}$ & $\left({ }^{\mathrm{T}} \mathrm{C}\right)$ & $\left(\begin{array}{c}5 \\
(00)\end{array}\right.$ & $\begin{array}{c}\sigma_{t} \\
(\mathrm{gm} / 1)\end{array}$ & $\begin{array}{c}\Delta D \\
(\mathrm{dyn}, \mathrm{m})\end{array}$ & $\begin{array}{c}\Delta D 1000-\triangle D \\
\text { (dyn. m) }\end{array}$ \\
\hline 00 & 26.87 & 35.31 & 23.02 & 0.000 & 1.735 \\
\hline 10 & 26.77 & 35.29 & 23.03 & 0.048 & 1.687 \\
\hline 20 & 26.70 & 35.29 & 23.05 & 0.097 & 1.639 \\
\hline 30 & 26.65 & 35.30 & 23.08 & 0.145 & 1.590 \\
\hline 50 & 26.60 & $35 \cdot 32$ & $23 \cdot 11$ & 0.241 & 1.494 \\
\hline 75 & 26.52 & 35.33 & 23.14 & 0.361 & $1 \cdot 375$ \\
\hline 100 & 26.13 & 35.41 & $23.3 ?$ & 0.473 & 1.257 \\
\hline 150 & 21.70 & 35.39 & 24.62 & 0.679 & 1.057 \\
\hline 200 & 13.88 & 35.02 & $26 \cdot 24$ & 0.210 & $C .925$ \\
\hline 250 & 11.83 & 34.86 & 26.53 & 0.896 & 0.840 \\
\hline 300 & 11.41 & 34.83 & 26.58 & 0.974 & 0.761 \\
\hline 400 & 10.12 & 34.75 & 26.76 & 1.121 & 0.615 \\
\hline 500 & 07.88 & 34.61 & 27.01 & I. 248 & 0.498 \\
\hline 600 & 06.83 & 34.55 & 27.11 & 1.359 & 0.377 \\
\hline 700 & 06.18 & 34.52 & 27.17 & 1.462 & 0.273 \\
\hline 800 & 05.62 & 34.52 & $27 \cdot 24$ & 1.560 & 0.176 \\
\hline 1000 & 04.48 & 34.53 & $27 \cdot 38$ & 1.735 & 0.000 \\
\hline
\end{tabular}


$M / V$ Hugh $M$. Smith: Cruise $14,2^{\circ} 00 ' S, 155^{\circ} 03^{\prime} W$, January 31 , 1952. Messenger time: $2124 \mathrm{GCT}$. Weather: 01 , cloud coverage 1. Wind: $020^{\circ}, 11 \mathrm{kt}$. Sea: < $1 \mathrm{ft}$. Wire angle: $35^{\circ}$

O B S R V E D

\begin{tabular}{|c|c|c|c|c|c|}
\hline $\begin{array}{c}\text { DEPTH } \\
(\mathrm{m})\end{array}$ & $\begin{array}{c}\mathrm{T} \\
\left({ }^{\circ} \mathrm{C}\right)\end{array}$ & $\begin{array}{c}\mathrm{S} \\
\left({ }^{\circ} / \mathrm{oo}\right)\end{array}$ & $\begin{array}{c}\sigma_{t} \\
(\mathrm{~g} / 1)\end{array}$ & $\begin{array}{c}\mathrm{O}_{2} \\
(\mathrm{ml} / 1)\end{array}$ & $\begin{array}{c}\mathrm{PO}_{4}-\mathrm{P} \\
(\mu \mathrm{g} \text { at } / 1)\end{array}$ \\
\hline 00 & 27.10 & 35.27 & 22.91 & \\
$0 \varepsilon$ & 26.87 & 35.26 & 22.92 & \\
25 & 26.68 & 35.26 & 23.04 & \\
49 & 26.72 & 35.29 & 23.05 & \\
95 & 26.66 & 35.34 & 23.10 & \\
191 & 14.17 & 35.12 & 26.26 & \\
286 & 11.51 & 34.83 & 26.57 & \\
379 & 10.76 & 34.79 & 26.67 & \\
476 & 08.93 & 34.69 & 26.91 & \\
571 & 07.73 & 34.61 & 27.03 & \\
771 & 05.61 & 34.52 & 27.24 & \\
771 & 05.65 & 34.52 & 27.24 & \\
973 & 04.54 & 34.56 & $>7.40$ & \\
1178 & 03.79 & 34.56 & 27.47 &
\end{tabular}

INTERPOLATED AND CALCULATED

\begin{tabular}{|c|c|c|c|c|c|}
\hline $\begin{array}{c}\text { DEPTH } \\
(\mathrm{m})\end{array}$ & $\left.{ }^{\mathrm{T}} \mathrm{C}\right)$ & $\begin{array}{c}\mathrm{S} \\
\left({ }^{\circ} / \mathrm{oo}\right)\end{array}$ & $\begin{array}{c}\sigma_{t} \\
(\mathrm{gm} / \mathrm{l})\end{array}$ & $\begin{array}{c}\Delta \mathrm{D} \\
(\mathrm{dyn} . \mathrm{m})\end{array}$ & $\begin{array}{c}\Delta \mathrm{D} 1000-\Delta \mathrm{D} \\
(\mathrm{dyn} . \mathrm{m})\end{array}$ \\
\hline
\end{tabular}

$\begin{array}{llllll}00 & 27.10 & 35.27 & 22.91 & 0.000 & 1.757 \\ 10 & 25.93 & 35.26 & 22.96 & 0.049 & 1.708 \\ 20 & 26.74 & 35.26 & 23.02 & 0.099 & 1.559 \\ 30 & 26.65 & 35.23 & 23.05 & 0.147 & 1.610 \\ 50 & 26.73 & 35.30 & 23.05 & 0.244 & 1.514 \\ 75 & 26.71 & 35.33 & 23.05 & 0.365 & 1.393 \\ 120 & 26.62 & 35.36 & 23.13 & 0.425 & 1.272 \\ 150 & 22.35 & 35.45 & 24.49 & 0.694 & 1.064 \\ 200 & 13.38 & 35.05 & 26.37 & 0.325 & 0.932 \\ 250 & 11.69 & 34.87 & 26.53 & 0.908 & 0.850 \\ 300 & 11.42 & 34.83 & 26.58 & 0.926 & 0.771 \\ 400 & 10.39 & 34.75 & 26.72 & 1.134 & 0.523 \\ 502 & 05.62 & 34.67 & 26.24 & 1.267 & 0.490 \\ 600 & 07.30 & 34.55 & 27.06 & 1.384 & 0.373 \\ 700 & 06.23 & 34.52 & 27.17 & 1.490 & 0.267 \\ 800 & 05.46 & 34.52 & 27.26 & 1.527 & 0.171 \\ 1200 & 04.41 & 34.56 & 27.41 & 1.757 & 0.000\end{array}$


M/V Hugh M. Smith: Cruise 14, $3^{\circ} 00^{\prime} S, 154^{\circ} 58^{\prime} \mathrm{W}$, February 1 , 1952. Messenger time: 0617 GCT. Weather: 21, cloud coverage not recorded. Wind: $000^{\circ}$, l l kt. Sea: < l ft. Wire angle: $27^{\circ}$

OBSER V E D

\begin{tabular}{|c|c|c|c|c|c|}
\hline $\begin{array}{c}\text { DEPTH } \\
(\mathrm{m})\end{array}$ & $\begin{array}{c}\mathrm{T} \\
\left({ }^{\circ} \mathrm{C}\right)\end{array}$ & $\begin{array}{c}\mathrm{S} \\
(\% / 00)\end{array}$ & $\begin{array}{c}\sigma_{\mathrm{t}} \\
(\mathrm{g} / \mathrm{l})\end{array}$ & $\begin{array}{c}\mathrm{O}_{2} \\
(\mathbf{m l} / \mathbf{l})\end{array}$ & $\begin{array}{c}\mathrm{PO}_{4}-\mathrm{P} \\
(\mu \mathrm{g} / \mathrm{t} / \mathrm{t})\end{array}$ \\
\hline 00 & 27.37 & 35.35 & 22.80 & \\
10 & 27.34 & 35.34 & 22.89 & \\
27 & 27.02 & 35.35 & 22.97 & \\
54 & 27.04 & 35.39 & 23.02 & \\
108 & 27.13 & 35.44 & 23.03 &
\end{tabular}

INTERPOLATED AND CALCULATED

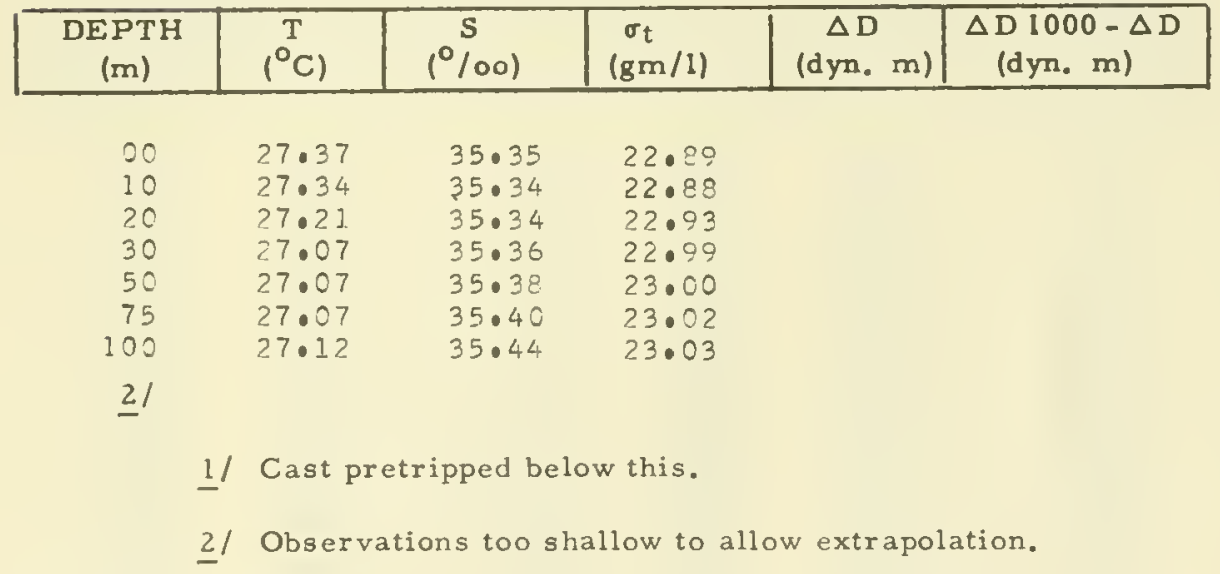


M/V Hugh M. Smith: Cruise $14,4^{\circ} 00$ 'S, $155^{\circ} 07^{\prime} \mathrm{W}$, February I, 1952. Messenger time: First cast $1548 \mathrm{GCT}$, second cast 1617 GCT. Weather: 15 , cloud coverage 7 . Wind: $040^{\circ}, 14 \mathrm{kt}$. Sea: $1-3 \mathrm{ft}$. Wire angle: First cast $17^{\circ}$, second cast $20^{\circ}$

O BSER VED

\begin{tabular}{c|c|c|c|}
\hline $\begin{array}{c}\text { DEPTH } \\
(\mathrm{m})\end{array}$ & $\begin{array}{c}\mathrm{T} \\
\left({ }^{\circ} \mathrm{C}\right)\end{array}$ & $\begin{array}{c}\mathrm{S} \\
(\% / 00)\end{array}$ & $\begin{array}{c}\sigma_{\mathrm{t}} \\
(\mathrm{g} / 1)\end{array}$ \\
\hline 00 & 27.65 & 35.55 & 22.94 \\
09 & 27.69 & 35.53 & 22.92 \\
28 & 27.64 & 35.52 & 22.92 \\
56 & 27.54 & 35.53 & 22.96 \\
\hline 112 & 27.60 & 35.56 & 22.96 \\
222 & 10.26 & 35.46 & 25.58 \\
330 & 10.41 & 34.76 & 26.71 \\
434 & 03.74 & 34.65 & 26.91 \\
541 & 07.48 & 34.59 & 27.05 \\
646 & 06.64 & 34.54 & 27.13 \\
861 & 05.18 & 34.52 & 27.30 \\
861 & 05.22 & 34.52 & 27.29 \\
1072 & 04.23 & 34.54 & 27.42 \\
1280 & 03.56 & 34.56 & 27.50
\end{tabular}

INTERPOLATED AND CALCULATED

\begin{tabular}{|c|c|c|c|c|c|}
\hline $\begin{array}{c}\text { DEPTH } \\
(\mathrm{m})\end{array}$ & $\begin{array}{c}\mathrm{T} \\
\left({ }^{\circ} \mathrm{C}\right)\end{array}$ & $\begin{array}{c}\mathrm{S} \\
(\% / 00)\end{array}$ & $\begin{array}{c}\sigma_{\mathrm{t}} \\
(\mathrm{gm} / \mathrm{l})\end{array}$ & $\begin{array}{c}\Delta \mathrm{D} \\
(\mathrm{dyn} . \mathrm{m})\end{array}$ & $\begin{array}{c}\Delta \mathrm{D} 1000-\Delta \mathrm{D} \\
(\mathrm{dyn} . \mathrm{m})\end{array}$ \\
\hline
\end{tabular}

$\begin{array}{rlllll}00 & 27.65 & 35.55 & 22.94 & 0.000 & 1.873 \\ 10 & 27.68 & 35.52 & 22.91 & 0.049 & 1.824 \\ 20 & 27.68 & 35.52 & 22.91 & 0.099 & 1.774 \\ 30 & 27.63 & 35.52 & 22.93 & 0.149 & 1.724 \\ 50 & 27.55 & 35.52 & 22.95 & 0.248 & 1.625 \\ 75 & 27.56 & 35.54 & 22.96 & 0.371 & 1.502 \\ 100 & 27.58 & 35.55 & 22.96 & 0.495 & 1.378 \\ 150 & 26.60 & 35.71 & 23.40 & 0.734 & 1.139 \\ 200 & 20.82 & 35.60 & 25.02 & 0.924 & 0.949 \\ 250 & 13.42 & 35.04 & 26.36 & 1.044 & 0.829 \\ 300 & 11.19 & 34.83 & 26.63 & 1.125 & 0.748 \\ 400 & 09.22 & 34.68 & 26.85 & 1.265 & 0.608 \\ 500 & 07.27 & 34.62 & 27.02 & 1.387 & 0.486 \\ 500 & 06.92 & 34.56 & 27.11 & 1.498 & 0.375 \\ 700 & 06.20 & 34.53 & 27.18 & 1.501 & 0.272 \\ 800 & 05.50 & 34.52 & 27.26 & 1.698 & 0.175 \\ 1000 & 04.56 & 34.53 & 27.37 & 1.873 & 0.000\end{array}$




\section{STATION 14}

M/V Hugh M. Smith: Cruise 14, $4^{\circ} 58^{\prime} \mathrm{S}, 155^{\circ} 00^{\prime} \mathrm{W}$, February 2 , 1952. Messenger time: $0441 \mathrm{GCT}$. Weather: 02, cloud coverage 6. Wind: $060^{\circ}, 18 \mathrm{kt}$. Sea: $3-5 \mathrm{ft}$. Wire angle: $32^{\circ}$

OBSER VED

\begin{tabular}{|c|c|c|c|c|c|}
\hline $\begin{array}{c}\text { DEPTH } \\
\text { (m) }\end{array}$ & $\left(\begin{array}{c}\mathrm{T} \\
\left({ }^{\circ} \mathrm{C}\right)\end{array}\right.$ & $\begin{array}{c}5 \\
(0 / 00)\end{array}$ & $\begin{array}{l}\sigma_{t} \\
(g / 1)\end{array}$ & $\begin{array}{c}\mathrm{O}_{2} \\
(\mathrm{ml} / 1)\end{array}$ & $\begin{array}{c}\mathrm{PO}_{4}-\mathrm{P} \\
(\mu \mathrm{g} \text { at } / 1)\end{array}$ \\
\hline 00 & 27.79 & 35.45 & $22 \cdot 32$ & & \\
\hline 09 & 27.84 & $35 \cdot 47$ & 22.82 & & \\
\hline 26 & 27.72 & $35 \cdot 43$ & 22.83 & & \\
\hline 51 & 27.66 & 35.48 & $22 \cdot 89$ & & \\
\hline 98 & 27.62 & 35.56 & 22.96 & & \\
\hline 191 & 21.10 & 35.89 & $25 \cdot 15$ & & \\
\hline 283 & 11.20 & 34.8211 & 26.63 & & \\
\hline 373 & 09.67 & $34 \cdot 70^{-}$ & $26 \cdot 79$ & & \\
\hline 467 & 08.51 & 34.63 & $26 \cdot 92$ & & \\
\hline 560 & 07.56 & 34.58 & 27.03 & & \\
\hline 753 & $05.9 \mathrm{C}$ & 34.51 & 27.20 & & \\
\hline 758 & 05.95 & 34.51 & $27 \cdot 20$ & & \\
\hline 952 & 04.59 & 34.51 & $27 \cdot 35$ & & \\
\hline 1149 & 03.99 & 34.54 & $27 \cdot 44$ & & \\
\hline
\end{tabular}

INTERPOLATED AND CALCULATED

\begin{tabular}{|c|c|c|c|c|c|}
\hline $\begin{array}{c}\text { DEPTH } \\
(\mathrm{m})\end{array}$ & $\left({ }^{\circ} \mathrm{C}\right)$ & $\begin{array}{c}5 \\
(\% 00)\end{array}$ & $\begin{array}{c}\sigma_{t} \\
(g \mathrm{~m} / 1)\end{array}$ & $\begin{array}{c}\Delta D \\
(\mathrm{dyn}, \mathrm{m})\end{array}$ & $\begin{array}{l}\Delta D 1000- \\
\text { (dyn. m) }\end{array}$ \\
\hline 00 & 27.79 & 35.45 & $22 \cdot \varepsilon 2$ & 0.000 & 1.842 \\
\hline 10 & 27.84 & $35 \cdot 47$ & 22.82 & 0.050 & 1.791 \\
\hline 20 & 27.74 & 35.45 & $22 \cdot \varepsilon 4$ & 0.101 & $1 \cdot 741$ \\
\hline 30 & $27.7 C$ & 35.44 & 22.84 & 0.151 & 1.691 \\
\hline 50 & 27.66 & 35.48 & 22.59 & 0.252 & 1.590 \\
\hline 75 & 27.64 & 35.50 & $22 \cdot 91$ & 0.377 & 1.465 \\
\hline 100 & 27.62 & 35.57 & $22 \cdot 96$ & 0.501 & $1 \cdot 340$ \\
\hline 150 & 26.25 & 35.98 & 23.71 & 0.732 & 1.109 \\
\hline 200 & $1 \varepsilon .52$ & 35.63 & $25 \cdot 64$ & 0.900 & 0.942 \\
\hline 250 & 12.62 & 34.97 & $26 \cdot 46$ & 1.002 & 0.839 \\
\hline 300 & $10 . \varepsilon 4$ & 34.79 & $26 \cdot 66$ & 1.080 & 0.762 \\
\hline 400 & 09.32 & 34.63 & $26 \cdot 23$ & 1.219 & 0.623 \\
\hline 500 & 00.14 & 34.61 & 26.97 & $1 \cdot 344$ & 0.457 \\
\hline 600 & 07.17 & 34.56 & 27.07 & 1.459 & 0.383 \\
\hline 700 & 06.34 & 34.52 & $27 \cdot 15$ & 1.566 & 0.276 \\
\hline 800 & 05.00 & $34 \cdot 51$ & $27 \cdot 24$ & 1.665 & 0.177 \\
\hline & 04.47 & 34.52 & $27 \cdot 33$ & 1.042 & \\
\hline
\end{tabular}

1/ This sample may possibly have been accidentally contaminated, but appears to be good. 
$\mathrm{M} / \mathrm{V}$ Hugh M. Smith: Cruise 14, $5^{\circ} 54^{\prime} \mathrm{S}, 155^{\circ} 03^{\prime} \mathrm{W}$, February 2 , 1952. Messenger time: 1411 GCT. Weather: 02, cloud coverage 2. Wind: $060^{\circ}, 18 \mathrm{kt}$. Sea: $3-5 \mathrm{ft}$. Wire angle: $32^{\circ}$

OBSER V E D

\begin{tabular}{|c|c|c|c|c|c|}
\hline $\begin{array}{c}\text { DEPTH } \\
(\mathrm{m})\end{array}$ & $\begin{array}{c}\mathrm{T} \\
\left({ }^{\circ} \mathrm{C}\right)\end{array}$ & $\begin{array}{c}\mathrm{S} \\
(\% / 00)\end{array}$ & $\begin{array}{c}\sigma_{\mathrm{t}} \\
(\mathrm{g} / 1)\end{array}$ & $\begin{array}{c}\mathrm{O}_{2} \\
(\mathrm{ml} / 1)\end{array}$ & $\begin{array}{c}\mathrm{PO}_{4}-\mathrm{P} \\
(\mu \mathrm{g} / / 1)\end{array}$ \\
\hline 00 & 27.79 & 35.52 & 22.87 & \\
09 & 27.82 & 35.54 & 22.88 & \\
26 & 27.78 & 35.54 & 22.89 & \\
51 & 27.81 & 35.55 & 22.89 & \\
102 & 28.07 & 35.76 & 22.96 & \\
204 & 21.94 & 36.09 & 25.08 & \\
305 & 11.46 & 34.83 & 26.58 & \\
404 & 08.88 & 34.67 & 26.89 & \\
507 & 07.84 & 34.58 & 26.99 & \\
608 & 07.04 & 34.54 & 27.07 & \\
822 & 05.45 & 34.52 & 27.26 & \\
1033 & 04.30 & 34.54 & 27.41 & \\
1240 & 03.66 & 34.58 & 27.51 &
\end{tabular}

INTERPOLATED AND CALCULATED

\begin{tabular}{|c|c|c|c|c|c|}
\hline $\begin{array}{c}\text { DEPTH } \\
(\mathrm{m})\end{array}$ & $\begin{array}{c}\mathrm{T} \\
\left({ }^{\circ} \mathrm{C}\right)\end{array}$ & $\begin{array}{c}\mathrm{S} \\
(\% / \mathrm{oo})\end{array}$ & $\begin{array}{c}\sigma_{\mathrm{t}} \\
(\mathrm{gm} / 1)\end{array}$ & $\begin{array}{c}\Delta \mathrm{D} \\
(\mathrm{dyn} . \mathrm{m})\end{array}$ & $\begin{array}{c}\Delta \mathrm{D} 1000-\Delta \mathrm{D} \\
\text { (dyn. m) }\end{array}$ \\
\hline
\end{tabular}

$\begin{array}{rlllll}00 & 27.79 & 35.52 & 22.87 & 0.000 & 1.898 \\ 10 & 27.80 & 35.54 & 22.89 & 0.050 & 1.848 \\ 20 & 27.80 & 35.54 & 22.89 & 0.100 & 1.798 \\ 30 & 27.79 & 35.54 & 22.89 & 0.150 & 1.748 \\ 50 & 27.81 & 35.55 & 22.89 & 0.250 & 1.648 \\ 75 & 28.13 & 35.65 & 22.86 & 0.375 & 1.523 \\ 100 & 28.06 & 35.76 & 22.97 & 0.500 & 1.398 \\ 150 & 27.22 & 36.02 & 23.43 & 0.739 & 1.160 \\ 200 & 22.32 & 36.10 & 24.98 & 0.928 & 0.969 \\ 250 & 15.18 & 35.30 & 26.18 & 1.054 & 0.844 \\ 300 & 11.64 & 34.56 & 26.56 & 1.141 & 0.757 \\ 400 & 08.93 & 34.68 & 26.90 & 1.281 & 0.617 \\ 500 & 07.94 & 34.58 & 26.97 & 1.403 & 0.495 \\ 600 & 07.10 & 34.54 & 27.06 & 1.517 & 0.380 \\ 700 & 06.33 & 34.52 & 27.15 & 1.624 & 0.274 \\ 800 & 05.60 & 34.52 & 27.24 & 1.723 & 0.175 \\ 1000 & 04.49 & 34.54 & 27.39 & 1.898 & 0.000\end{array}$


M/V Hugh M. Smith: Cruise 14, $6^{\circ} 50^{\prime} S, 155^{\circ} 05^{\prime} W$, February 2, 1952. Messenger time: 2322 GCT. Weather: 02, cloud coverage 5. Wind: $020^{\circ}, 13 \mathrm{kt}$. Sea: $3-5 \mathrm{ft}$. Wire angle: $12^{\circ}$

OBSER VED

\begin{tabular}{|c|c|c|c|c|c|}
\hline $\begin{array}{c}\text { DEPTH } \\
(\mathrm{m})\end{array}$ & $\begin{array}{c}\mathrm{T} \\
\left({ }^{\circ} \mathrm{C}\right)\end{array}$ & $\begin{array}{c}\mathrm{S} \\
(\% / 00)\end{array}$ & $\begin{array}{c}\sigma_{t} \\
(\mathrm{~g} / 1)\end{array}$ & $\begin{array}{c}\mathrm{O}_{2} \\
(\mathrm{ml} / \mathrm{l})\end{array}$ & $\begin{array}{c}\mathrm{PO}_{4}-\mathrm{P} \\
(\mu \mathrm{g} \text { at } / 1)\end{array}$ \\
\hline 00 & 28.49 & 35.75 & 22.82 & \\
10 & 29.48 & 35.76 & 22.83 & \\
30 & 28.40 & 35.76 & 22.86 & \\
50 & 28.35 & 35.76 & 22.87 & \\
118 & 28.19 & 35.76 & 22.93 & \\
233 & 19.69 & 35.92 & 25.56 & \\
347 & 09.04 & 34.72 & 26.76 & \\
458 & 08.22 & 34.61 & 26.95 & \\
573 & 06.02 & 34.53 & 27.09 & \\
682 & 06.02 & 34.52 & 27.19 & \\
910 & 04.81 & 3.4 .52 & 27.34 & \\
1132 & 04.10 & 34.54 & 27.43 & \\
1344 & 03.54 & 34.54 & 27.49 &
\end{tabular}

INTERPOLATED AND CALCULATED

\begin{tabular}{|c|c|c|c|c|c|}
\hline $\begin{array}{c}\text { DEPTH } \\
(\mathrm{m})\end{array}$ & $\begin{array}{c}\mathrm{T} \\
\left({ }^{\circ} \mathrm{C}\right)\end{array}$ & $\begin{array}{c}\mathrm{S} \\
(\% / 00)\end{array}$ & $\begin{array}{c}\sigma_{\mathrm{t}} \\
(\mathrm{gm} / \mathrm{l})\end{array}$ & $\begin{array}{c}\Delta \mathrm{D} \\
(\mathrm{dyn} . \mathrm{m})\end{array}$ & $\begin{array}{c}\Delta \mathrm{D} 1000-\Delta \mathrm{D} \\
(\mathrm{dyn} . \mathrm{m})\end{array}$ \\
\hline
\end{tabular}

$\begin{array}{rlllll}00 & 28.49 & 35.75 & 22.82 & 0.000 & 1.911 \\ 10 & 28.49 & 35.76 & 22.23 & 0.050 & 1.860 \\ 20 & 28.44 & 35.76 & 22.84 & 0.101 & 1.810 \\ 30 & 28.40 & 35.76 & 22.96 & 0.151 & 1.760 \\ 50 & 28.39 & 35.76 & 22.86 & 0.252 & 1.659 \\ 75 & 28.30 & 35.76 & 22.89 & 0.377 & 1.534 \\ 100 & 28.25 & 35.76 & 22.90 & 0.503 & 1.408 \\ 150 & 27.18 & 36.03 & 23.46 & 0.742 & 1.169 \\ 200 & 23.00 & 36.13 & 24.81 & 0.936 & 0.975 \\ 250 & 17.44 & 35.62 & 25.90 & 1.072 & 0.839 \\ 300 & 12.42 & 34.95 & 26.49 & 1.168 & 0.743 \\ 400 & 09.05 & 34.66 & 26.35 & 1.314 & 0.597 \\ 500 & 07.68 & 34.57 & 27.00 & 1.436 & 0.475 \\ 600 & 06.63 & 34.53 & 27.12 & 1.546 & 0.365 \\ 700 & 05.87 & 34.52 & 27.21 & 1.647 & 0.264 \\ 800 & 05.25 & 34.52 & 27.29 & 1.740 & 0.171 \\ 1000 & 04.50 & 34.53 & 27.38 & 1.911 & 0.000\end{array}$


M/V Hugh M. Smith: Cruise $14,7^{\circ} 56$ 'S, $179^{\circ} 53^{\prime} \mathrm{W}$, February 15 . 1952. Messenger time: $1236 \mathrm{GCT}$. Weather: 03, cloud coverage 3. Wind: $040^{\circ}, 8 \mathrm{kt}$. Sea: $1-3 \mathrm{ft}$. Wire angle: $13^{\circ}$

OBSER VED

\begin{tabular}{|c|c|c|c|c|c|}
\hline $\begin{array}{c}\text { DEPTH } \\
\text { (m) }\end{array}$ & $\frac{\mathrm{T}}{\left({ }^{\circ} \mathrm{C}\right)}$ & $\begin{array}{c}S \\
(0 / 00)\end{array}$ & $\begin{array}{c}\sigma_{\mathrm{t}} \\
(\mathrm{g} / 1)\end{array}$ & $\begin{array}{c}\mathrm{O}_{2} \\
(\mathrm{ml} / \mathrm{l})\end{array}$ & $\begin{array}{c}\mathrm{PO}_{4}-\mathrm{P} \\
(\mu \mathrm{g} \text { at } / 1)\end{array}$ \\
\hline 00 & 29.58 & 34.90 & $21 \cdot 81$ & & 0.35 \\
\hline 09 & 29.60 & 34.89 & 21.80 & & 0.44 \\
\hline 23 & 29.42 & 34.90 & 21.87 & & 0.46 \\
\hline 52 & 29.36 & $35 \cdot 15$ & 22.08 & & 0.45 \\
\hline 104 & $28 \cdot 36$ & 35.60 & $22 \cdot 75$ & & 0.69 \\
\hline 207 & $22 \cdot 86$ & 36.07 & 24.30 & & 0.94 \\
\hline 311 & 13.54 & 34.97 & $26 \cdot 28$ & & $1 \cdot 76$ \\
\hline 420 & 09.23 & 34.68 & 26.85 & & $2 \cdot 26$ \\
\hline 525 & 07.52 & $34 \cdot 56$ & 27.02 & & $2 \cdot 41$ \\
\hline 630 & 06.54 & 34.52 & $27 \cdot 13$ & & $2 \cdot 47$ \\
\hline 835 & 05.11 & $34 \cdot 50$ & 27.29 & & $2 \cdot 72$ \\
\hline 835 & 05.15 & 34.50 & 27.28 & & \\
\hline 1044 & 04.28 & 34.53 & 27.40 & & $2 \cdot 81$ \\
\hline 1245 & 03.72 & $34 \cdot 53$ & $27 \cdot 46$ & & 2.66 \\
\hline
\end{tabular}

INTERPOLATED AND CALCULATED

\begin{tabular}{|c|c|c|c|c|c|}
\hline $\begin{array}{c}\text { DEPTH } \\
\text { (m) }\end{array}$ & $\left({ }^{\circ} \mathrm{C}\right)$ & $\begin{array}{c}5 \\
(\% / 00)\end{array}$ & $\begin{array}{c}\sigma_{t} \\
(\mathrm{gm} / 1)\end{array}$ & $\begin{array}{c}\Delta D \\
(\mathrm{dyn}, \mathrm{m})\end{array}$ & $\begin{array}{c}\Delta D 1000-\Delta D \\
\text { (dyn, m) }\end{array}$ \\
\hline$O C$ & 29.58 & 34.90 & 21.82 & 0.000 & 2.037 \\
\hline 10 & 29.60 & 34.89 & 21.80 & 0.060 & 1.976 \\
\hline 20 & 29.50 & 34.90 & 21.84 & 0.120 & 1.916 \\
\hline 30 & 29.41 & 34.94 & 21.90 & 0.180 & 1.857 \\
\hline 50 & $29 \cdot 37$ & 35.13 & 22.06 & 0.297 & 1.739 \\
\hline 75 & 29.18 & 35.29 & $22 \cdot 24$ & 0.440 & $1 \cdot 596$ \\
\hline 100 & $28 \cdot 49$ & 35.55 & $22 \cdot 67$ & 0.576 & 1.460 \\
\hline 150 & 26.20 & 35.96 & 23.71 & 0.814 & $1 \cdot 222$ \\
\hline 200 & $23 \cdot 42$ & 36.09 & 24.66 & 1.006 & 1.031 \\
\hline 250 & 18.59 & 35.67 & 25.65 & 1.152 & 0.885 \\
\hline 300 & $14 \cdot 40$ & 35.09 & $26 \cdot 19$ & 1.261 & 0.775 \\
\hline 400 & 09.72 & 34.71 & $26 \cdot 79$ & 1.426 & 0.611 \\
\hline 500 & 07.24 & 34.58 & 26.99 & 1.552 & 0.485 \\
\hline 600 & 06.79 & 34.52 & 27.09 & 1.664 & 0.372 \\
\hline 700 & 06.00 & 34.51 & 27.19 & 1.768 & 0.269 \\
\hline 800 & 05.32 & 34.50 & $27 \cdot 26$ & 1.863 & 0.174 \\
\hline 1000 & 04.45 & 34.52 & $27 \cdot 38$ & 2.037 & 0.000 \\
\hline
\end{tabular}


M/V Hugh M. Smith: Cruise 14, $7^{\circ}$ Ol'S, $179^{\circ} 49^{\prime} W$. February 15 , 1952. Messengertime: $2219 \mathrm{GCT}$. Weather: 02, cloud coverage 4. Wind: $050^{\circ}, 12 \mathrm{kt}$. Sea: $1-3 \mathrm{ft}$. Wire angle: $17^{\circ}$

OBSER VED

\begin{tabular}{|c|c|c|c|c|c|}
\hline $\begin{array}{c}\text { DEPTH } \\
\text { (m) }\end{array}$ & $\begin{array}{c}\mathrm{T} \\
\left({ }^{\circ} \mathrm{C}\right)\end{array}$ & $\begin{array}{c}5 \\
(0 / 00)\end{array}$ & $\begin{array}{c}\sigma_{t} \\
(g / 1)\end{array}$ & $\begin{array}{c}\mathrm{O}_{2} \\
(\mathrm{ml} / \mathrm{l})\end{array}$ & $\begin{array}{c}\mathrm{PO}_{4}-\mathrm{P} \\
(\mu \mathrm{g} \text { at } / 1) \\
\end{array}$ \\
\hline$C O$ & 29.32 & 34.91 & 21.91 & & 0.38 \\
\hline 10 & 29.23 & 34.90 & 21.91 & & 0.39 \\
\hline 24 & $29 \cdot 24$ & $34 \cdot 94$ & 21.96 & & 0.40 \\
\hline 52 & 79.4 .2 & 35.10 & 22.02 & & 0.44 \\
\hline 102 & 28.99 & 35.51 & 22.47 & & 0.66 \\
\hline 205 & 23.96 & $36 \cdot 19$ & $24 \cdot 58$ & & 0.86 \\
\hline 308 & $14 \cdot 12$ & 35.06 & $26 \cdot 23$ & & 1.74 \\
\hline 416 & 09.10 & 34.66 & $26 \cdot 86$ & & $2 \cdot 25$ \\
\hline 519 & 07.45 & 34.57 & 27.04 & & $2 \cdot 40$ \\
\hline 823 & 05.19 & 34.50 & $27 \cdot 28$ & & 2.68 \\
\hline 823 & 05.22 & 34.50 & $27 \cdot 27$ & & \\
\hline 621 & 06.43 & $34 \cdot 51$ & 27.13 & & $2 \cdot 47$ \\
\hline 1026 & 04.26 & 34.52 & 27.40 & & $2 \cdot 80$ \\
\hline 1223 & 03.70 & 34.55 & 27.48 & & $2 \cdot 82$ \\
\hline
\end{tabular}

INTERPOLATED AND CALCULATED

\begin{tabular}{|c|c|c|c|c|c|}
\hline $\begin{array}{l}\text { DEPTH } \\
(\mathrm{m})\end{array}$ & $\left({ }^{\circ} \mathrm{C}\right)$ & $\begin{array}{c}5 \\
(0 / 0)\end{array}$ & $\begin{array}{c}\sigma_{\mathrm{t}} \\
(\mathrm{gm} / 1)\end{array}$ & $\begin{array}{c}\Delta D \\
\text { (dyn. m) }\end{array}$ & $\begin{array}{c}\Delta D 1000-\triangle D \\
\text { (dyn.m) }\end{array}$ \\
\hline 00 & 29.32 & 34.91 & 21.91 & 0.000 & 2.084 \\
\hline 10 & 29.28 & 34.90 & $21 \cdot 92$ & 0.059 & 2.025 \\
\hline 20 & 29.26 & 34.92 & 21.94 & 0.118 & 1.966 \\
\hline 30 & 29.23 & 34.97 & 21.99 & 0.177 & 1.907 \\
\hline 50 & 29.40 & 35.08 & 22.01 & 0.294 & 1.790 \\
\hline 75 & 29.25 & $35 \cdot 33$ & 22.25 & 0.437 & 1.646 \\
\hline 100 & 29.00 & 35.49 & $22 \cdot 45$ & 0.576 & 1.508 \\
\hline 150 & 28.08 & 35.77 & 22.97 & 0.837 & 1.247 \\
\hline 200 & $24 \cdot 45$ & 36.19 & $24 \cdot 42$ & 1.052 & 1.031 \\
\hline 250 & 18.20 & 35.72 & $25 \cdot 64$ & $1 \cdot 204$ & 0.879 \\
\hline 300 & 14.80 & $35 \cdot 15$ & $26 \cdot 15$ & $1 \cdot 315$ & 0.769 \\
\hline 400 & 09.55 & 34.69 & 26.80 & $1 \cdot 481$ & 0.603 \\
\hline 500 & 07.67 & $34 \cdot 58$ & 27.01 & 1.605 & 0.478 \\
\hline 600 & 06.63 & 34.52 & $27 \cdot 11$ & 1.715 & 0.368 \\
\hline 700 & 05.90 & 34.51 & 27.20 & 1.817 & 0.267 \\
\hline 800 & 05.32 & $34 \cdot 51$ & $27 \cdot 27$ & 1.912 & 0.172 \\
\hline 1000 & 04.39 & 34.52 & 27.39 & 2.084 & 0.000 \\
\hline
\end{tabular}


M/V Hugh M. Smith: Cruise 14, $6^{\circ} 04^{\prime} \mathrm{S}, 179^{\circ} 59^{\prime} \mathrm{W}$, February 16 , 1952. Messenger time: $0727 \mathrm{GCT}$. Weather: 02 , cloud coverage 4. Wind: $050^{\circ}, 17 \mathrm{kt}$. Sea: $3-5 \mathrm{ft}$. Wire angle: $28^{\circ}$

OBSER VED

\begin{tabular}{|c|c|c|c|c|c|}
\hline $\begin{array}{c}\text { DEPTH } \\
\text { (m) }\end{array}$ & $\begin{array}{c}\mathrm{T} \\
\left({ }^{\circ} \mathrm{C}\right)\end{array}$ & $\begin{array}{c}5 \\
(0 / 00)\end{array}$ & $\begin{array}{c}\sigma_{t} \\
(g / 1)\end{array}$ & $\begin{array}{c}\mathrm{O}_{2} \\
(\mathrm{ml} / \mathrm{l})\end{array}$ & $\begin{array}{c}\mathrm{PO}_{4}-\mathrm{P} \\
(\mu \mathrm{g} \text { at } / 1)\end{array}$ \\
\hline 00 & 29.37 & 35.47 & $22 \cdot 31$ & & 0.47 \\
\hline OE & 29.40 & 35.44 & $22 \cdot 28$ & & 0.49 \\
\hline 26 & $29 \cdot 34$ & 35.45 & $22 \cdot 31$ & & 0.52 \\
\hline 50 & 29.12 & 35.46 & $22 \cdot 39$ & & 0.65 \\
\hline 101 & $28 . \$ 1$ & 35.58 & 22.58 & & 0.67 \\
\hline 198 & 23.85 & 36.25 & 24.65 & & 0.86 \\
\hline 297 & $14 \cdot 15$ & $35 \cdot 11$ & $26 \cdot 26$ & & 1.76 \\
\hline 392 & 10.34 & $34.7 \mathrm{c}$ & $26 \cdot 74$ & & $2 \cdot 12$ \\
\hline 493 & 08.20 & 34.63 & 26.97 & & $2 \cdot 28$ \\
\hline 592 & 07.16 & 34.57 & 27.08 & & $2 \cdot 37$ \\
\hline 802 & 05.32 & 34.52 & 27.28 & & 2.63 \\
\hline 802 & 05.35 & $34 \cdot 52$ & 27.27 & & \\
\hline 1013 & $04 \cdot 36$ & 34.53 & 27.40 & & $2 \cdot 70$ \\
\hline 1224 & 03.72 & 34.57 & 27.49 & & 2.79 \\
\hline
\end{tabular}

INTERPOLATED AND CALCULATED

\begin{tabular}{|c|c|c|c|c|c|}
\hline $\begin{array}{c}\text { DEPTH } \\
(\mathrm{m})\end{array}$ & $\begin{array}{c}\mathrm{T} \\
\left({ }^{\circ} \mathrm{C}\right)\end{array}$ & $\begin{array}{c}\mathrm{S} \\
(\% / 00)\end{array}$ & $\begin{array}{c}\sigma_{t} \\
(\mathrm{gm} / \mathrm{l})\end{array}$ & $\begin{array}{c}\Delta \mathrm{D} \\
(\mathrm{dyn} . \mathrm{m})\end{array}$ & $\begin{array}{c}\Delta \mathrm{D} 1000-\Delta \mathrm{D} \\
(\mathrm{dyn} . \mathrm{m})\end{array}$ \\
\hline
\end{tabular}

$\begin{array}{llllll}00 & 29.37 & 35.47 & 22.31 & 0.000 & 2.010 \\ 10 & 29.40 & 35.44 & 22.28 & 0.055 & 1.955 \\ 20 & 29.39 & 35.44 & 22.29 & 0.111 & 1.899 \\ 30 & 29.28 & 35.45 & 22.33 & 0.167 & 1.844 \\ 50 & 29.12 & 35.46 & 22.39 & 0.277 & 1.734 \\ 75 & 29.00 & 35.50 & 22.46 & 0.413 & 1.597 \\ 100 & 28.82 & 35.58 & 22.59 & 0.547 & 1.463 \\ 150 & 26.91 & 36.02 & 23.53 & 0.792 & 1.218 \\ 200 & 23.82 & 36.25 & 24.66 & 0.988 & 1.022 \\ 250 & 18.00 & 35.61 & 25.76 & 1.131 & 0.879 \\ 300 & 14.01 & 35.10 & 26.26 & 1.236 & 0.774 \\ 400 & 10.12 & 34.76 & 26.76 & 1.398 & 0.612 \\ 500 & 08.11 & 34.62 & 26.98 & 1.527 & 0.483 \\ 600 & 07.09 & 34.57 & 27.09 & 1.640 & 0.370 \\ 700 & 06.19 & 34.54 & 27.19 & 1.744 & 0.266 \\ 800 & 05.36 & 34.52 & 27.27 & 1.839 & 0.171 \\ 1000 & 04.39 & 34.53 & 27.39 & 2.010 & 0.000\end{array}$


STATION 20

$\mathrm{M} / \mathrm{V}$ Hugh $M$. Smith: Cruise $14,5^{\circ} 031 \mathrm{~S}, 179^{\circ} 59^{\prime} \mathrm{W}$, February 16 , 1952. Messenger time: $1705 \mathrm{GCT}$. Weather: 02, cloud coverage not recorded. Wind: $060^{\circ}, 14 \mathrm{kt}$. Sea: $1-3 \mathrm{ft}$. Wire angle: $22^{\circ}$

OBSER VED

\begin{tabular}{|c|c|c|c|c|c|}
\hline $\begin{array}{c}\text { DEPTH } \\
(\mathrm{m})\end{array}$ & $\begin{array}{c}\mathrm{T} \\
\left({ }^{\circ} \mathrm{C}\right)\end{array}$ & $\begin{array}{c}\mathrm{S} \\
(\% / 00)\end{array}$ & $\begin{array}{c}\sigma_{\mathrm{t}} \\
(\mathrm{g} / 1)\end{array}$ & $\begin{array}{c}\mathrm{O}_{2} \\
(\mathrm{ml} / 1)\end{array}$ & $\begin{array}{c}\mathrm{PO}_{4}-\mathrm{P} \\
(\mu \mathrm{g} \text { at } / 1)\end{array}$ \\
\hline
\end{tabular}

$\begin{array}{rrrrr}00 & 29.14 & 35.51 & 22.42 & 0.51 \\ 09 & 29.18 & 35.47 & 22.38 & 0.51 \\ 23 & 29.15 & 35.47 & 22.39 & 0.58 \\ 50 & 29.18 & 35.50 & 22.40 & 0.58 \\ 99 & 29.03 & 35.60 & 22.52 & 0.61 \\ 149 & 28.01 & 35.62 & 22.88 & 0.84 \\ 197 & 24.54 & 36.26 & 24.45 & 0.90 \\ 246 & 18.00 & 35.52 & 25.69 & 1.35 \\ 295 & 12.58 & 34.95 & 26.46 & 1.97 \\ 349 & 9.90 & 34.73 & 26.78 & 2.25 \\ 398 & 9.10 & 34.69 & 26.88 & 2.32 \\ 398 & 9.05 & 34.69 & 26.89 & 2.43 \\ 496 & 8.00 & 34.62 & 27.00 & 2.56 \\ 597 & 7.12 & 34.57 & 27.09 & \end{array}$

\section{STATION 21}

$M / V$ Hugh $M$. Smith: Cruise $14,3^{\circ} 59^{\prime} S, 179^{\circ} 59^{\prime} W$, February 17, 1952. Messenger time: $0242 \mathrm{GCT}$. Weather: 01 , cloud coverage 2. Wind: $070^{\circ}, 16 \mathrm{kt}$. Sea: $3-5 \mathrm{ft}$. Wire angle: $22^{\circ}$

O BSER VED

\begin{tabular}{|c|c|c|c|c|c|}
\hline $\begin{array}{c}\text { DEPTH } \\
(\mathrm{m})\end{array}$ & $\left.\sigma^{\mathrm{T}} \mathrm{C}\right)$ & $\left.\sigma^{\circ} / 00\right)$ & $\begin{array}{c}\sigma_{\mathrm{t}} \\
(\mathrm{g} / 1)\end{array}$ & $\begin{array}{c}\mathrm{O}_{2} \\
(\mathrm{ml} / 1)\end{array}$ & $\begin{array}{c}\mathrm{PO}_{4}-\mathrm{P} \\
(\mu \mathrm{g} \text { at } / 1)\end{array}$ \\
\hline
\end{tabular}

$\begin{array}{rrrrr}00 & 28.76 & 35.59 & 22.61 & 0.64 \\ 09 & 28.74 & 35.60 & 22.62 & 0.64 \\ 29 & 28.59 & 35.59 & 22.66 & 0.64 \\ 55 & 28.59 & 35.58 & 22.66 & 0.64 \\ 111 & 28.62 & 35.59 & 22.65 & 0.64 \\ 164 & 27.59 & 35.67 & 23.05 & 0.82 \\ 218 & 21.76 & 36.01 & 25.10 & 1.01 \\ 274 & 13.55 & 35.03 & 26.32 & 1.95 \\ 328 & 10.35 & 34.78 & 26.74 & 2.23 \\ 382 & 9.30 & 34.67 & 26.83 & 2.32 \\ 433 & 8.78 & 34.65 & 26.90 & 2.44 \\ 433 & 8.82 & 34.65 & 26.89 & 2.61 \\ 543 & 7.60 & 34.60 & 27.04 & 2.71 \\ 651 & 6.54 & 34.53 & 27.13 & \end{array}$


STATION 22

$\mathrm{M} / \mathrm{V}$ Hugh $M$. Smith: Cruise $14,2^{\circ} 56^{\prime} \mathrm{S}, 180^{\circ} 00^{\prime} \mathrm{W}$, February 17 , 1952. Messenger time: $1212 \mathrm{GCT}$. Weather: 02, cloud coverage not recorded. Wind: $050^{\circ}, 20 \mathrm{kt}$. Sea: $3-5 \mathrm{ft}$. Wire angle: $37^{\circ}$

O BSER V E D

\begin{tabular}{|c|c|c|c|c|c|}
\hline $\begin{array}{c}\text { DEPTH } \\
(\mathrm{m})\end{array}$ & $\begin{array}{c}\mathrm{T} \\
\left({ }^{\circ} \mathrm{C}\right)\end{array}$ & $\begin{array}{c}\mathrm{S} \\
(\% / 00)\end{array}$ & $\begin{array}{c}\sigma_{\mathrm{t}} \\
(\mathrm{g} / 1)\end{array}$ & $\begin{array}{c}\mathrm{O}_{2} \\
(\mathrm{ml} / 1)\end{array}$ & $\begin{array}{c}\mathrm{PO}_{4}-\mathrm{P} \\
(\mu \mathrm{g} \mathrm{at} / 1)\end{array}$ \\
\hline
\end{tabular}

$\begin{array}{rrrr}00 & 28.17 & 35.55 & 22.77 \\ 09 & 28.20 & 35.55 & 22.76 \\ 25 & 28.17 & 35.53 & 22.76 \\ 49 & 28.16 & 35.56 & 22.78 \\ 92 & 28.20 & 35.57 & 22.78 \\ 132 & 27.90 & 35.56 & 22.87 \\ 171 & 25.71 & 35.74 & 23.70 \\ 206 & 18.04 & 35.52 & 25.68 \\ 241 & 12.82 & 34.98 & 26.43 \\ 280 & 11.32 & 34.86 & 26.63 \\ 315 & 10.33 & 34.76 & 26.73 \\ 315 & 10.36 & 34.76 & 26.72 \\ 399 & 9.12 & 34.66 & 26.85 \\ 488 & 7.98 & 34.61 & 26.99\end{array}$

1/ Phosphate analysis on this station was faulty.

\section{STATION 23}

$M / V$ Hugh $M$. Smith: Cruise $14,1^{\circ} 52^{\prime} \mathrm{S}, 179^{\circ} 54^{\prime} \mathrm{E}$, February 17 , 1952. Messenger time: $2234 \mathrm{GCT}$. Weather: 02, cloud coverage 2. Wind: $060^{\circ}, 10 \mathrm{kt}$. Sea: $1-3 \mathrm{ft}$. Wire angle: $43^{\circ}$

OBSER VED

\begin{tabular}{|c|c|c|c|c|c|}
\hline $\begin{array}{c}\text { DEPTH } \\
(\mathrm{m})\end{array}$ & $\left.{ }^{\mathrm{T}} \mathrm{C}\right)$ & $\begin{array}{c}\mathrm{S} \\
(\% / \mathrm{oo})\end{array}$ & $\begin{array}{c}\sigma_{\mathrm{t}} \\
(\mathrm{g} / \mathrm{l})\end{array}$ & $\begin{array}{c}\mathrm{O}_{2} \\
(\mathrm{ml} / 1)\end{array}$ & $\begin{array}{c}\mathrm{PO}_{4}-\mathrm{P} \\
(\mu \mathrm{g} \text { at } / 1)\end{array}$ \\
\hline
\end{tabular}

$\begin{array}{rrrrr}00 & 28.00 & 35.53 & 22.81 & 0.68 \\ 11 & 27.92 & 35.54 & 22.85 & 0.68 \\ 24 & 27.88 & 35.53 & 22.85 & 0.70 \\ 51 & 27.90 & 35.53 & 22.85 & 0.70 \\ 96 & 27.92 & 35.53 & 22.84 & 0.69 \\ 135 & 27.92 & 35.57 & 22.87 & 0.71 \\ 167 & 25.64 & 35.77 & 23.74 & 0.99 \\ 195 & 16.90 & 35.36 & 25.83 & 1.59 \\ 228 & 13.64 & 35.04 & 26.31 & 1.76 \\ 265 & 11.41 & 34.83 & 26.58 & 1.89 \\ 298 & 10.62 & 34.77 & 26.68 & 2.25 \\ 298 & 10.66 & 34.77 & 26.68 & \\ 370 & 9.85 & 34.75 & 26.80 & 2.41 \\ 469 & 9.04 & 34.69 & 26.89 & \end{array}$


STATION 24

M/V Hugh M. Smith: Cruise 14, $0^{\circ} 59^{\prime} S, 179^{\circ} 57^{\prime} \mathrm{E}$, February 18 , 1952. Messenger time: $0702 \mathrm{GCT}$. Weather: 02, cloud coverage 2. Wind: $070^{\circ}, 13 \mathrm{kt}$. Sea: $1-3 \mathrm{ft}$. Wire angle: $1 / 35^{\circ}$

OBSER V E D

\begin{tabular}{|c|c|c|c|c|c|}
\hline $\begin{array}{c}\text { DEPTH } \\
(\mathrm{m})\end{array}$ & $\begin{array}{c}\mathrm{T} \\
\left({ }^{\circ} \mathrm{C}\right)\end{array}$ & $\begin{array}{c}\mathrm{S} \\
(\% / 00)\end{array}$ & $\begin{array}{c}\sigma_{\mathrm{t}} \\
(\mathrm{g} / 1)\end{array}$ & $\begin{array}{c}\mathrm{O}_{2} \\
(\mathrm{ml} / 1)\end{array}$ & $\begin{array}{c}\mathrm{PO}_{4}-\mathrm{P} \\
(\mu \mathrm{g} \text { at } / 1)\end{array}$ \\
\hline
\end{tabular}

$\begin{array}{lllll}04 & 27.74 & 35.44 & 22.83 & 0.84 \\ 17 & 27.62 & 35.44 & 22.87 & 0.92 \\ 34 & 27.55 & 35.44 & 22.89 & 0.88 \\ 69 & 27.56 & 35.44 & 22.89 & 0.96 \\ 134 & 25.93 & 35.57 & 23.50 & 1.15 \\ 197 & 16.53 & 35.12 & 25.73 & 1.57 \\ 261 & 11.74 & 34.86 & 26.55 & 2.13 \\ 321 & 10.92 & 34.77 & 26.63 & 2.50 \\ 386 & 10.25 & 34.75 & 26.73 & 2.61 \\ 455 & 9.31 & 34.70 & 26.85 & 2.74 \\ 520 & 8.42 & 34.65 & 26.95 & 2.85 \\ 520 & 8.45 & 34.65 & 26.95 & 3.12 \\ 647 & 6.44 & 34.56 & 27.17 & 3.13 \\ 787 & 5.24 & 34.55 & 27.31 & \end{array}$

1/ Underway to reduce wire angle.

\section{STATION 25}

$M / V$ Hugh M. Smith: Cruise 14, $0^{\circ} 02^{\prime} N, 179^{\circ} 59^{\prime} E$, February 18 , 1952. Messenger time: $1648 \mathrm{GCT}$. Weather: 02, cloud coverage not recorded. Wind: $060^{\circ}, 9 \mathrm{kt}$. Sea: $1-3 \mathrm{ft}$. Wire angle: $1 / 27^{\circ}$

O BSER VED

\begin{tabular}{|c|c|c|c|c|c|}
\hline $\begin{array}{c}\text { DEPTH } \\
(\mathrm{m})\end{array}$ & $\left({ }^{\circ} \mathrm{C}\right)$ & $\left(\begin{array}{c}5 \\
0\end{array}\right.$ & $\begin{array}{l}\sigma_{t} \\
(g / 1)\end{array}$ & $\begin{array}{c}\mathrm{O}_{2} \\
(\mathrm{ml} / \mathrm{l}) \\
\end{array}$ & $\begin{array}{c}\mathrm{PO}_{4}-\mathrm{P} \\
(\mu \mathrm{g} \text { at } / 1)\end{array}$ \\
\hline $\begin{array}{l}05 \\
20 \\
39 \\
76 \\
146 \\
217 \\
289 \\
355 \\
426 \\
503 \\
576 \\
576 \\
715 \\
866\end{array}$ & $\begin{array}{r}27.45 \\
27.48 \\
27.39 \\
27.16 \\
22.26 \\
15.87 \\
11.70 \\
10.88 \\
9.60 \\
8.52 \\
7.39 \\
7.43 \\
5.83 \\
4.82\end{array}$ & $\begin{array}{l}35.49 \\
35.50 \\
35.48 \\
35.48 \\
35.25 \\
35.13 \\
34.85 \\
34.79 \\
34.72 \\
34.67 \\
34.61 \\
34.61 \\
34.54 \\
34.53\end{array}$ & $\begin{array}{l}22 \cdot 96 \\
22 \cdot 96 \\
22 \cdot 97 \\
23 \cdot 05 \\
24 \cdot 35 \\
25 \cdot 90 \\
26 \cdot 55 \\
26 \cdot 65 \\
26 \cdot 79 \\
26 \cdot 95 \\
27 \cdot 08 \\
27 \cdot 07 \\
27 \cdot 23 \\
27.35\end{array}$ & & $\begin{array}{l}0.92 \\
0.95 \\
0.96 \\
0.99 \\
1.21 \\
1.56 \\
2.30 \\
2.62 \\
2.88 \\
3.05 \\
3.17 \\
3.16 \\
3.24\end{array}$ \\
\hline
\end{tabular}

1) Underway to reduce wire angle. 
STATION 26

M/V Hugh M. Smith: Cruise 14, $1^{\circ} 03^{\prime} \mathrm{N}, 179^{\circ} 58^{\prime} \mathrm{W}$, February 19 , 1952. Messenger time: $0346 \mathrm{GCT}$. Weather: 03, cloud coverage 8 . Wind: $090^{\circ}, 13 \mathrm{kt}$. Sea: $1-3 \mathrm{ft}$. Wire angle: $49^{\circ}$

O BS E R E D

\begin{tabular}{|c|c|c|c|c|c|}
\hline $\begin{array}{c}\text { DEPTH } \\
\text { (m) }\end{array}$ & $\begin{array}{c}\mathrm{T} \\
\left({ }^{\circ} \mathrm{C}\right)\end{array}$ & $\begin{array}{c}5 \\
(0 / 00)\end{array}$ & $\begin{array}{c}\sigma_{t} \\
(g / 1)\end{array}$ & $\begin{array}{c}\mathrm{O}_{2} \\
(\mathrm{ml} / \mathrm{l})\end{array}$ & $\begin{array}{l}\mathrm{PO}_{4-}- \\
\text { ( } \mu \mathrm{g} \text { at }\end{array}$ \\
\hline 04 & 28.03 & 35.49 & $22 \cdot 77$ & & 0.91 \\
\hline 15 & 27.58 & 35.47 & $22 \cdot 91$ & & 0.93 \\
\hline 26 & 27.50 & 35.47 & 22.93 & & 0.94 \\
\hline 49 & 27.50 & 35.48 & $22 \cdot 94$ & & 0.94 \\
\hline 88 & $27 \cdot 48$ & $35 \cdot 46$ & $22 \cdot 93$ & & 0.91 \\
\hline 121 & $27 \cdot 36$ & $35 \cdot 42$ & $22 \cdot 94$ & & 0.93 \\
\hline 148 & 26.63 & $35 \cdot 27$ & $23 \cdot 06$ & & 0.96 \\
\hline 172 & 19.37 & 34.88 & 24.85 & & 1.24 \\
\hline 200 & $16 \cdot 22$ & 35.08 & $25 \cdot 78$ & & 1.51 \\
\hline 233 & 12.52 & 34.69 & $26 \cdot 27$ & & 1.75 \\
\hline 266 & $11 \cdot 46$ & 34.30 & $26 \cdot 55$ & & 2.18 \\
\hline 333 & 10.66 & $34 \cdot 76$ & $26 \cdot 67$ & & 2.50 \\
\hline 430 & 9.36 & 34.69 & 26.84 & & 2.71 \\
\hline
\end{tabular}

\section{STATION 27}

M/V Hugh M. Smith: Cruise 14, $2^{\circ} 07^{\prime} \mathrm{N}, 179^{\circ} 57^{\prime} \mathrm{W}$, February 19 , 1952. Messenger time: $1336 \mathrm{GCT}$. Weather: 02, cloud coverage not recorded. Wind: $070^{\circ}, 14 \mathrm{kt}$. Sea: $3-5 \mathrm{ft}$. Wire angle: $1 / 25^{\circ}$

O BSER V E D

\begin{tabular}{|c|c|c|c|c|c|}
\hline $\begin{array}{c}\text { DEPTH } \\
\text { (m) }\end{array}$ & $\left({ }^{\circ} \mathrm{C}\right)$ & $\left.{ }^{\circ} / 00\right)$ & $\begin{array}{l}\sigma_{t} \\
(g / 1)\end{array}$ & $\begin{array}{c}\mathrm{O}_{2} \\
(\mathrm{ml} / \mathrm{l})\end{array}$ & $\begin{array}{l}\mathrm{PO}_{4}- \\
(\mu \mathrm{g} \text { at. }\end{array}$ \\
\hline 04 & 27.85 & 35.34 & 22.72 & & 0.82 \\
\hline 18 & 27.86 & 35.35 & 22.72 & & 0.85 \\
\hline 36 & 27.74 & $35 \cdot 34$ & 22.76 & & 0.87 \\
\hline 74 & 27.68 & $35 \cdot 36$ & $22 \cdot 79$ & & 0.88 \\
\hline 144 & $26 \cdot 72$ & $35 \cdot 22$ & $22 \cdot 98$ & & 1.03 \\
\hline 214 & 11.72 & 34.63 & 26.37 & & 2.09 \\
\hline 283 & 10.96 & 34.79 & 26.64 & & $2 \cdot 45$ \\
\hline 348 & $10 \cdot 30$ & $34 \cdot 75$ & $26 \cdot 73$ & & 2.58 \\
\hline 418 & 9.72 & 34.73 & 26.81 & & 2.74 \\
\hline 493 & 8.90 & 34.66 & 26.89 & & 2.83 \\
\hline 564 & 8.14 & 34.61 & 26.97 & & $2 \cdot 84$ \\
\hline 564 & 8.19 & 34.61 & 26.97 & & \\
\hline 703 & $6 \cdot 14$ & 34.53 & 27.19 & & 3.26 \\
\hline 855 & 4.92 & 34.53 & 27.33 & & $3 \cdot 28$ \\
\hline
\end{tabular}

1) Underway to reduce wire angle. 


\section{STATION 28}

$\mathrm{M} / \mathrm{V}$ Hugh $\mathrm{M}$. Smith: Cruise $14,3^{\circ} 06^{\prime} \mathrm{N}, 179^{\circ} 57^{\prime} \mathrm{W}$, February 19 , 1952. Messenger time: $2237 \mathrm{GCT}$. Weather: 02 , cloud coverage 8 . Wind: $100^{\circ}, 7 \mathrm{kt}$. Sea: $1-3 \mathrm{ft}$. Wire angle: $32^{\circ}$

OBSER VED

\begin{tabular}{|c|c|c|c|c|c|}
\hline $\begin{array}{c}\text { DEPTH } \\
(\mathrm{m})\end{array}$ & $\begin{array}{c}\mathrm{T} \\
\left({ }^{\circ} \mathrm{C}\right)\end{array}$ & $\begin{array}{c}\mathrm{S} \\
(\% / 00)\end{array}$ & $\begin{array}{c}\sigma_{\mathrm{t}} \\
(\mathrm{g} / 1)\end{array}$ & $\begin{array}{c}\mathrm{O}_{2} \\
(\mathrm{ml} / 1)\end{array}$ & $\begin{array}{c}\mathrm{PO}_{4}-\mathrm{P} \\
(\mu \mathrm{g} \text { at } / 1)\end{array}$ \\
\hline
\end{tabular}

$\begin{array}{rrrrr}00 & 28.11 & 35.22 & 22.54 & 0.78 \\ 13 & 28.03 & 35.23 & 22.58 & 0.81 \\ 30 & 28.00 & 35.27 & 22.62 & 0.86 \\ 63 & 28.02 & 35.31 & 22.64 & 0.91 \\ 122 & 27.94 & 35.29 & 22.65 & 0.91 \\ 176 & 22.86 & 35.00 & 23.99 & 0.96 \\ 231 & 12.43 & 34.55 & 26.17 & 2.16 \\ 284 & 9.96 & 34.64 & 26.70 & 2.74 \\ 343 & 9.12 & 34.68 & 26.87 & 2.80 \\ 409 & 8.49 & 34.63 & 26.93 & 2.77 \\ 474 & 7.93 & 34.61 & 27.00 & 36 \\ 474 & 7.99 & 34.61 & 26.99 & 3.10 \\ 607 & 6.96 & 34.56 & 27.10 & \end{array}$

STATION 29

$M / V$ Hugh $M$. Smith: Cruise $14,4^{\circ} 05^{\prime} N, 179^{\circ} 55^{\prime} W$, February 20 , 1952. Messenger time: $0741 \mathrm{GCT}$. Weather: 63, cloud coverage 10. Wind: $150^{\circ}, 11 \mathrm{kt}$. Sea: $3-5 \mathrm{ft}$. Wire angle: $15^{\circ}$

O BSER VED

\begin{tabular}{|c|c|c|c|c|c|}
\hline $\begin{array}{c}\text { DEPTH } \\
\text { (m) }\end{array}$ & $\left({ }^{\mathrm{T}} \mathrm{C}\right)$ & $(0 / 00)$ & $\begin{array}{l}\sigma_{t} \\
(g / 1)\end{array}$ & $\begin{array}{c}\mathrm{O}_{2} \\
(\mathrm{ml} / \mathrm{l})\end{array}$ & $\begin{array}{l}\mathrm{PO}_{4-\mathrm{P}} \\
(\mu \mathrm{g} \text { at } / 1)\end{array}$ \\
\hline 00 & 27.93 & 34.89 & $22 \cdot 34$ & & 0.80 \\
\hline 10 & 28.09 & 35.23 & $22 \cdot 56$ & & 0.80 \\
\hline 29 & 28.01 & $35 \cdot 25$ & 22.60 & & 0.81 \\
\hline 59 & $28 \cdot 00$ & 35.25 & 22.60 & & 0.80 \\
\hline 115 & 28.00 & 35.25 & 22.60 & & 0.80 \\
\hline 173 & $27 \cdot 35$ & 35.17 & $22 \cdot 75$ & & 0.90 \\
\hline 230 & 11.81 & 34.55 & 26.29 & & 2.23 \\
\hline 289 & 9.26 & $34 \cdot 61$ & 26.79 & & 2.65 \\
\hline 343 & 8.66 & 34.61 & 26.89 & & 2.59 \\
\hline 400 & 8.12 & $34.6 C$ & $26 \cdot 96$ & & 2.74 \\
\hline 452 & 7.68 & 34.58 & 27.01 & & $2 \cdot 80$ \\
\hline 452 & 7.73 & 34.58 & 27.00 & & \\
\hline 569 & 6.86 & 34.56 & 27.11 & & 3.06 \\
\hline $68 \mathrm{C}$ & 6.07 & 34.55 & $27 \cdot 21$ & & 3.21 \\
\hline
\end{tabular}


STATION 30

$M / V$ Hugh M. Smith: Cruise 14, $5^{\circ} 066^{\prime} N, 179^{\circ} 50^{\prime} W$, February 20 , 1952. Messenger time: $1637 \mathrm{GCT}$. Weather: '51, cloud coverage not recorded. Wind: $130^{\circ}, 5 \mathrm{kt}$. Sea: $1-3 \mathrm{ft}$. Wire angle: $05^{\circ}$

O BSER VED

\begin{tabular}{|c|c|c|c|c|c|}
\hline $\begin{array}{c}\text { DEPTH } \\
(\mathrm{m})\end{array}$ & $\begin{array}{c}\mathrm{T} \\
\left({ }^{\circ} \mathrm{C}\right)\end{array}$ & $\begin{array}{c}\mathrm{S} \\
(\% / \mathrm{oo})\end{array}$ & $\begin{array}{c}\sigma_{\mathrm{t}} \\
(\mathrm{g} / \mathrm{l})\end{array}$ & $\begin{array}{c}\mathrm{O}_{2} \\
(\mathrm{ml} / 1)\end{array}$ & $\begin{array}{c}\mathrm{PO}_{4}-\mathrm{P} \\
(\mu \mathrm{g} \text { at } / 1)\end{array}$ \\
\hline
\end{tabular}

$\begin{array}{rrrrr}00 & 28.17 & 34.88 & 22.27 & 0.65 \\ 10 & 28.22 & 34.89 & 22.26 & 0.61 \\ 31 & 28.22 & 34.92 & 22.28 & 0.71 \\ 60 & 28.20 & 34.96 & 22.32 & 0.77 \\ 120 & 26.96 & 35.22 & 22.92 & 0.99 \\ 177 & 19.88 & 34.88 & 24.72 & 1.22 \\ 236 & 11.34 & 34.60 & 26.42 & 2.55 \\ 293 & 9.68 & 34.64 & 26.74 & 2.82 \\ 351 & 8.96 & 34.65 & 26.87 & 2.93 \\ 408 & 8.42 & 34.64 & 26.95 & 2.82 \\ 461 & 8.07 & 34.59 & 26.96 & 2.83 \\ 461 & 8.12 & 34.55 & 26.95 & 3.08 \\ 579 & 7.28 & 34.58 & 27.07 & 3.14 \\ 691 & 6.42 & 34.53 & 27.15 & \end{array}$

STATION 31

M/V Hugh M. Smith: Cruise 14, $6^{\circ} 06^{\prime} \mathrm{N}, 179^{\circ} 44^{\prime} \mathrm{W}$, February 21 , 1952. Messenger time: $0141 \mathrm{GCT}$. Weather: 63, cloud coverage 10. Wind: $130^{\circ}, 12 \mathrm{kt}$. Sea: $3-5 \mathrm{ft}$. Wire angle: $05^{\circ}$

OBSER VED

\begin{tabular}{|c|c|c|c|c|c|}
\hline $\begin{array}{c}\text { DEPTH } \\
(\mathrm{m})\end{array}$ & $\begin{array}{c}\mathrm{T} \\
\left({ }^{\circ} \mathrm{C}\right)\end{array}$ & $\begin{array}{c}\mathrm{S} \\
\left({ }^{\circ} / \mathrm{oo}\right)\end{array}$ & $\begin{array}{c}\sigma_{\mathrm{t}} \\
(\mathrm{g} / \mathrm{h})\end{array}$ & $\begin{array}{c}\mathrm{O}_{2} \\
(\mathrm{ml} / 1)\end{array}$ & $\begin{array}{c}\mathrm{PO}_{4}-\mathrm{P} \\
(\mu \mathrm{g} / \mathrm{at} / 1)\end{array}$ \\
\hline 00 & 28.07 & 34.69 & 22.16 & & 0.63 \\
10 & 28.10 & 34.69 & 22.15 & & 0.54 \\
25 & 28.08 & 34.71 & 22.17 & & 0.58 \\
55 & 28.12 & 34.70 & 22.15 & & 0.64 \\
110 & 27.58 & 34.71 & 22.33 & & 0.64 \\
165 & 15.78 & 34.63 & 25.53 & & 2.52 \\
218 & 11.14 & 34.58 & 26.44 & & 2.84 \\
271 & 9.88 & 34.65 & 26.72 & & 2.89 \\
324 & 9.24 & 34.65 & 26.83 & & 2.94 \\
382 & 8.66 & 34.62 & 26.89 & & 3.17 \\
436 & 8.35 & 34.62 & 26.94 & & 3.32 \\
436 & 8.39 & 34.62 & 26.94 & & \\
543 & 7.37 & 34.56 & 27.04 & & \\
650 & 6.50 & 34.53 & 27.14 & & \\
\end{tabular}


M/V Hugh M. Smith: Cruise 14, $7^{\circ} 04^{\prime} \mathrm{N}, 179^{\circ} 59^{\prime} \mathrm{E}$, February 21 , 1952. Messenger time: $1040 \mathrm{GCT}$. Weather: 02, cloud coverage not recorded. Wind: $080^{\circ}, 13 \mathrm{kt}$. Sea: $3-5 \mathrm{ft}$. Wire angle: $03^{\circ}$ OBSER VED

\begin{tabular}{|c|c|c|c|c|c|}
\hline $\begin{array}{c}\text { DEPTH } \\
\text { (m) }\end{array}$ & $\left({ }^{\circ} \mathrm{C}\right)$ & $\begin{array}{c}5 \\
(0 / 00)\end{array}$ & $\begin{array}{l}\sigma_{\mathrm{t}} \\
(g / 1)\end{array}$ & $\begin{array}{c}\mathrm{O}_{2} \\
(\mathrm{ml} / \mathrm{l})\end{array}$ & $\begin{array}{l}\mathrm{PO}_{4} \\
(\mu \mathrm{g} \text { at }\end{array}$ \\
\hline 00 & $27.92 \frac{1}{1}$ & 34.70 & $22.22 \frac{1}{/}$ & & 0.49 \\
\hline 10 & $27 \cdot 96$ & 34.68 & $22 \cdot 19$ & & 0.57 \\
\hline 30 & $27 \cdot 92$ & 34.70 & $22 \cdot 22$ & & 0.51 \\
\hline 60 & 27.94 & $34 \cdot 70$ & $22 \cdot 21$ & & 0.56 \\
\hline 120 & 20.53 & 34.87 & $24 \cdot 54$ & & 0.73 \\
\hline 180 & 13.00 & 34.51 & $26 \cdot 03$ & & 1.92 \\
\hline 240 & 10.37 & 34.63 & 26.60 & & $2 \cdot 74$ \\
\hline 297 & 9.32 & 34.64 & 26.80 & & 2.79 \\
\hline 355 & 8.77 & 34.63 & 26.89 & & 2.82 \\
\hline 413 & 8.32 & 34.60 & $26 \cdot 93$ & & $2 \cdot 78$ \\
\hline 466 & 7.90 & $34 \cdot 60$ & 27.00 & & $2 \cdot 86$ \\
\hline 466 & 7.96 & 34.60 & $26 \cdot 99$ & & \\
\hline 521 & 7.07 & 34.55 & 27.03 & & 3.04 \\
\hline 693 & 6.28 & 34.52 & $27 \cdot 16$ & & $3 \cdot 16$ \\
\hline
\end{tabular}

1) Auxiliary of surface bottle thermometer (a single protected thermometer was used in the surface bottle only) was broken. An average value from nearby bottles in the rack was used for correction; temperature and sigma-t may be in error by 0.01 .

\section{STATION 33}

$\mathrm{M} / \mathrm{V}$ Hugh M. Smith: Cruise $14,8^{\circ} 03^{\prime} \mathrm{N}, 179^{\circ} 58^{\prime} \mathrm{W}$, February 21 , 1952. Messenger time: $1936 \mathrm{GCT}$. Weather: 14, cloud coverage 8 . Wind: $070^{\circ}, 6 \mathrm{kt}$. Sea: $3-5 \mathrm{ft}$. Wire angle: $05^{\circ}$

\section{OBSER VED}

\begin{tabular}{|c|c|c|c|c|c|}
\hline $\begin{array}{c}\text { DEPTH } \\
(\mathrm{m})\end{array}$ & $\begin{array}{c}\mathrm{T} \\
\left({ }^{\circ} \mathrm{C}\right)\end{array}$ & $\begin{array}{c}\mathrm{S} \\
(\% / 00)\end{array}$ & $\begin{array}{c}\sigma_{\mathrm{t}} \\
(\mathrm{g} / \mathrm{l})\end{array}$ & $\begin{array}{c}\mathrm{O}_{2} \\
(\mathrm{ml} / \mathrm{l})\end{array}$ & $\begin{array}{c}\mathrm{PO}_{4}-\mathrm{P} \\
(\mu \mathrm{g} \text { at } / 1)\end{array}$ \\
\hline
\end{tabular}

$\begin{array}{rrrrr}05 & 27.52 & 34.70 & 22.35 & 0.62 \\ 25 & 27.54 & 34.69 & 22.33 & 0.60 \\ 31 & 27.50 & 34.70 & 22.35 & 0.62 \\ 60 & 27.53 & 34.69 & 22.34 & 0.66 \\ 113 & 19.28 & 34.80 & 24.81 & 0.82 \\ 165 & 12.17 & 34.47 & 26.17 & 2.29 \\ 219 & 10.56 & 34.58 & 26.55 & 2.86 \\ 272 & 10.00 & 34.66 & 26.71 & 2.95 \\ 325 & 9.53 & 34.66 & 26.78 & 3.08 \\ 383 & 9.03 & 34.65 & 26.86 & 3.08 \\ 437 & 8.51 & 34.61 & 26.91 & 3.17 \\ 437 & 8.55 & 34.61 & 26.90 & 3.38 \\ 544 & 7.48 & 34.56 & 27.03 & \\ 650 & 6.56 & 34.55 & 27.14 & \end{array}$




\section{STATION 34}

$\mathrm{M} / \mathrm{V}$ Hugh M. Smith: Cruise $14,8^{\circ} 58^{\prime} \mathrm{N}, 179^{\circ} 55^{\prime} \mathrm{W}$, February 22 , 1952. Messenger time: first cast 0355 GCT, second cast 0429 GCT. Weather: 20, cloud coverage 9. Wind: $050^{\circ}, 21 \mathrm{kt}$. Sea: $5-8 \mathrm{ft}$. Wire angle: first cast $25^{\circ}$, second cast $15^{\circ}$

OBSER VED

\begin{tabular}{|c|c|c|c|c|c|}
\hline $\begin{array}{c}\text { DEPTH } \\
(\mathrm{m})\end{array}$ & $\begin{array}{c}\mathrm{T} \\
\left({ }^{\circ} \mathrm{C}\right)\end{array}$ & $\begin{array}{c}\mathrm{S} \\
(\% / 00)\end{array}$ & $\begin{array}{c}\sigma_{\mathrm{t}} \\
(\mathrm{g} / 1)\end{array}$ & $\begin{array}{c}\mathrm{O}_{2} \\
(\mathrm{ml} / 1)\end{array}$ & $\begin{array}{c}\mathrm{PO}_{4}-\mathrm{P} \\
(\mu \mathrm{g} \mathrm{at} / 1)\end{array}$ \\
\hline
\end{tabular}

\begin{tabular}{lllll}
00 & 27.05 & 34.43 & 22.34 & 0.50 \\
10 & 27.08 & 34.49 & 22.33 & 0.51 \\
30 & 27.02 & 34.49 & 22.35 & 0.54 \\
59 & 27.03 & 34.49 & 22.35 & 0.58 \\
\hline 116 & 16.20 & 34.63 & 25.44 & 1.36 \\
\hline 162 & 11.34 & 34.52 & 26.36 & 2.50 \\
215 & 10.45 & 34.69 & 26.65 & 2.90 \\
270 & 9.82 & 34.55 & 26.73 & 2.94 \\
322 & 9.30 & 34.64 & 26.81 & 3.01 \\
376 & 8.38 & 34.62 & 26.86 & 2.95 \\
427 & 8.47 & 34.58 & 26.89 & 3.05 \\
427 & 8.52 & 34.58 & 26.88 & 3.21 \\
540 & 7.47 & 34.56 & 27.03 & 3.23 \\
650 & 6.72 & 34.53 & 27.11 &
\end{tabular}

1/ This one bottle is second cast.

\section{STATION 35}

M/V Hugh M. Smith: Cruise 14, $5^{\circ} 00^{\prime} S, 168^{\circ} 57^{\prime} \mathrm{W}$, March 2, 1952. Messenger time: 0409 GCT. Weather: 02, cloud coverage 2 . Wind: $060^{\circ}, 9 \mathrm{kt}$. Sea: $<1 \mathrm{ft}$. Wire angle: $18^{\circ}$

O B S R V E D

\begin{tabular}{|c|c|c|c|c|c|}
\hline $\begin{array}{c}\text { DEPTH } \\
\text { (m) }\end{array}$ & $\left({ }^{\circ} \mathrm{C}\right)$ & $\left.{ }^{\mathrm{S}} / 00\right)$ & $\begin{array}{l}\sigma_{t} \\
(g / 1)\end{array}$ & $\begin{array}{c}\mathrm{O}_{2} \\
(\mathrm{ml} / 1)\end{array}$ & $\begin{array}{c}\mathrm{PO}_{4}-\mathrm{P} \\
(\mu \mathrm{g} \text { at } / 1)\end{array}$ \\
\hline 00 & 28.89 & 35.58 & 22.56 & & 0.48 \\
\hline 10 & $28 \cdot 38$ & $35 \cdot 56$ & 22.71 & & 0.51 \\
\hline 23 & 28.31 & 35.58 & 22.75 & & 0.50 \\
\hline 52 & $28 \cdot 29$ & 35.60 & $22 \cdot 77$ & & 0.50 \\
\hline 103 & 27.70 & 35.53 & 22.91 & & 0.66 \\
\hline 153 & 27.52 & 35.67 & 23.07 & & 0.70 \\
\hline 202 & 22.54 & 36.09 & 24.91 & & 0.84 \\
\hline 251 & 15.90 & 35.27 & 26.00 & & 1.49 \\
\hline 301 & 12.02 & 34.92 & $26 \cdot 54$ & & 1.92 \\
\hline 355 & 9.83 & 34.75 & 26.80 & & $2 \cdot 13$ \\
\hline 404 & 9.08 & 34.69 & $26 \cdot 88$ & & $2 \cdot 30$ \\
\hline 404 & 9.12 & 34.69 & $26 \cdot 88$ & & \\
\hline 503 & 6.08 & 34.62 & 26.98 & & $2 \cdot 36$ \\
\hline 603 & 6.70 & 34.52 & $27 \cdot 10$ & & 2.48 \\
\hline
\end{tabular}




\section{STATION 36}

M/V Hugh M. Smith: Cruise $14,4^{\circ} 02^{\prime} \mathrm{S}, 168^{\circ} 58^{\prime} \mathrm{W}$, March 2, 1952. Messenger time: $1254 \mathrm{GCT}$. Weather: 00, cloud coverage not recorded. Wind: $060^{\circ}, 13 \mathrm{kt}$. Sea: $<1 \mathrm{ft}$. Wire angle: $28^{\circ}$

OBSER VED

\begin{tabular}{|c|c|c|c|c|c|}
\hline $\begin{array}{c}\text { DEPTH } \\
(\mathrm{m})\end{array}$ & $\begin{array}{c}\mathrm{T} \\
\left({ }^{\circ} \mathrm{C}\right)\end{array}$ & $\begin{array}{c}\mathrm{S} \\
(0 / 00)\end{array}$ & $\begin{array}{c}\sigma_{\mathrm{t}} \\
(\mathrm{g} / 1)\end{array}$ & $\begin{array}{c}\mathrm{O}_{2} \\
(\mathrm{ml} / 1)\end{array}$ & $\begin{array}{c}\mathrm{PO}_{4}-\mathrm{P} \\
(\mu \mathrm{g} \text { at } / 1)\end{array}$ \\
\hline
\end{tabular}

$\begin{array}{rrrrr}00 & 27.94 & 35.46 & 22.78 & 0.55 \\ 09 & 27.96 & 35.46 & 22.77 & 0.55 \\ 27 & 27.90 & 35.47 & 22.80 & 0.55 \\ 52 & 27.84 & 35.48 & 22.83 & 0.55 \\ 101 & 27.85 & 35.50 & 22.84 & 0.57 \\ 147 & 27.34 & 25.82 & 23.25 & 0.70 \\ 193 & 17.56 & 35.45 & 25.74 & 1.25 \\ 240 & 12.44 & 34.97 & 26.50 & 1.91 \\ 287 & 10.48 & 34.79 & 26.72 & 2.11 \\ 334 & 9.34 & 34.72 & 26.78 & 2.20 \\ 377 & 9.29 & 34.70 & 26.86 & 2.24 \\ 377 & 9.33 & 34.70 & 26.85 & 2.41 \\ 474 & 8.18 & 34.62 & 26.97 & 2.65 \\ 569 & 7.31 & 34.53 & 27.06 & \end{array}$

\section{STATION 37}

M/V Hugh M. Smith: Cruise 14, $3^{\circ} 04 ' S, 168^{\circ} 59^{\prime} \mathrm{W}$, March 2, 1952. Messenger time: $2231 \mathrm{GCT}$. Weather: 02, cloud coverage 3 . Wind: $070^{\circ}, 14 \mathrm{kt}$. Sea: $1-3 \mathrm{ft}$. Wire angle: $45^{\circ}$

O BSER VE D

\begin{tabular}{|c|c|c|c|c|c|}
\hline $\begin{array}{c}\text { DEPTH } \\
(\mathrm{m})\end{array}$ & $\left.{ }^{\mathrm{T}} \mathrm{C}\right)$ & $\left.\rho^{\circ} / 00\right)$ & $\begin{array}{c}\sigma_{\mathrm{t}} \\
(\mathrm{g} / 1)\end{array}$ & $\begin{array}{c}\mathrm{O}_{2} \\
(\mathrm{ml} / 1)\end{array}$ & $\begin{array}{c}\mathrm{PO}_{4}-\mathrm{P} \\
(\mu \mathrm{g} \text { at } / 1)\end{array}$ \\
\hline
\end{tabular}

$\begin{array}{rrrrr}00 & 27.84 & 35.50 & 22.84 & 0.59 \\ 12 & 27.80 & 35.50 & 22.86 & 0.57 \\ 26 & 27.72 & 35.48 & 22.87 & 0.56 \\ 51 & 27.65 & 35.49 & 22.90 & 0.57 \\ 95 & 27.62 & 35.47 & 22.89 & 0.57 \\ 133 & 27.30 & 35.50 & 23.08 & 0.69 \\ 166 & 18.97 & 35.56 & 25.47 & 1.23 \\ 196 & 15.00 & 35.19 & 26.14 & 1.64 \\ 230 & 12.32 & 34.93 & 26.49 & 1.97 \\ 263 & 10.50 & 34.77 & 26.70 & 2.14 \\ 295 & 10.16 & 34.76 & 26.76 & \\ 295 & 10.19 & 34.76 & 26.75 & 2.07 \\ 359 & 9.87 & 34.76 & 26.81 & 2.28 \\ 452 & 8.76 & 34.66 & 26.91 & \end{array}$




\section{STATION 38}

$M / V$ Hugh M. Smith: Cruise 14, $2^{\circ} 03^{\prime} S, 168^{\circ} 57^{\prime} \mathrm{W}$, March 3, 1952.

Messenger time: first cast $0829 \mathrm{GCT}, 5$ econd cast $0910 \mathrm{GCT}$. Weather: 00 , cloud coyerage not recorded. Wind: $080^{\circ}, 14 \mathrm{kt}$. Sea: $3-5 \mathrm{ft}$. Wire angle: 1- first cast $45^{\circ}$, second Cast $45^{\circ}$

\begin{tabular}{|c|c|c|c|c|c|}
\hline $\begin{array}{c}\text { DEPTH } \\
(\mathrm{m})\end{array}$ & $\begin{array}{c}\mathrm{T} \\
\left({ }^{\circ} \mathrm{C}\right)\end{array}$ & $(\% / 00)$ & $\begin{array}{c}\sigma_{t} \\
(\mathrm{~g} / 1)\end{array}$ & $\begin{array}{c}\mathrm{O}_{2} \\
(\mathrm{ml} / 1)\end{array}$ & $\begin{array}{c}\mathrm{PO}_{4}-\mathrm{P} \\
(\mu \mathrm{g} \text { at } / 1)\end{array}$ \\
\hline
\end{tabular}

\begin{tabular}{rrrrr}
02 & 27.15 & 35.26 & 22.89 & 0.70 \\
13 & 27.18 & 35.26 & 22.88 & 0.71 \\
23 & 27.08 & 35.26 & 22.91 & 0.65 \\
55 & 27.00 & 35.28 & 22.95 & 0.65 \\
107 & 27.11 & 35.54 & 23.11 & 0.74 \\
\hline 161 & 18.45 & 35.51 & 25.57 & 1.31 \\
213 & 12.28 & 34.93 & 26.50 & 1.70 \\
264 & 11.24 & 34.80 & 26.59 & 2.04 \\
317 & 10.69 & 34.76 & 26.66 & 2.21 \\
373 & 10.10 & 34.73 & 26.74 & 2.16 \\
441 & 9.60 & 34.68 & 26.79 & 2.25 \\
554 & 8.50 & 34.64 & 26.93 & 2.44 \\
689 & 6.40 & 34.55 & 27.17 & 2.68
\end{tabular}

1/ Underway to reduce wire angle.

\section{STATION 39}

M/V Hugh M. Smith: Cruise $14,1^{\circ} 02^{\prime} S, 168^{\circ} 54^{\prime} \mathrm{W}$, March 3, 1952. Messenger time: $1901 \mathrm{GCT}$. Weather: 02 , cloud coverage 1 .

Wind: $090^{\circ}, 14 \mathrm{kt}$. Sea: $3-5 \mathrm{ft}$. Wire angle: $1 / 25^{\circ}$

\section{O BSER V E D}

\begin{tabular}{|c|c|c|c|c|c|}
\hline $\begin{array}{c}\text { DEPTH } \\
(\mathrm{m})\end{array}$ & $\left.\mathrm{\rho}^{\mathrm{T}} \mathrm{C}\right)$ & $(\% / \mathrm{oo})$ & $\begin{array}{c}\sigma_{\mathrm{t}} \\
(\mathrm{g} / 1)\end{array}$ & $\begin{array}{c}\mathrm{O}_{2} \\
(\mathrm{ml} / 1)\end{array}$ & $\begin{array}{c}\mathrm{PO}_{4}-\mathrm{P} \\
(\mu \mathrm{g} \text { at } / 1)\end{array}$ \\
\hline
\end{tabular}

$\begin{array}{rrrrr}02 & 26.39 & 35.26 & 22.97 & 0.69 \\ 15 & 26.92 & 35.25 & 22.95 & 0.72 \\ 34 & 26.39 & 35.27 & 22.93 & 0.66 \\ 69 & 26.56 & 35.31 & 23.02 & 0.67 \\ 134 & 21.50 & 35.66 & 24.88 & 1.02 \\ 199 & 13.10 & 35.00 & 26.39 & 1.49 \\ 266 & 11.59 & 34.83 & 26.55 & 1.89 \\ 327 & 10.84 & 34.78 & 26.65 & 2.23 \\ 395 & 9.98 & 34.74 & 26.77 & 2.21 \\ 457 & 9.20 & 34.69 & 26.86 & 2.22 \\ 534 & 8.34 & 34.63 & 26.95 & 2.43 \\ 534 & 8.39 & 34.63 & 26.94 & 2.61 \\ 657 & 6.32 & 34.56 & 27.19 & 2.68 \\ 792 & 5.41 & 34.53 & 27.28 & \end{array}$

1/ Underway to reduce wire angle. 
STATION 40

M/V Hugh M. Smith: Cruise 14, $0^{\circ} 011^{\prime} N, 169^{\circ} 00^{\prime} \mathrm{W}$, March 4, 1952. Messenger time: $0455 \mathrm{GCT}$. Weather: 02, cloud coverage 1.

Wind: $090^{\circ}, 16 \mathrm{kt}$. Sea: $3-5 \mathrm{ft}$. Wire angle: $1 / 45^{\circ}$

OBSER VED

\begin{tabular}{|c|c|c|c|c|c|}
\hline $\begin{array}{c}\text { DEPTH } \\
(\mathrm{m})\end{array}$ & $\begin{array}{c}\mathrm{T} \\
\left({ }^{\circ} \mathrm{C}\right)\end{array}$ & $\begin{array}{c}\mathrm{S} \\
(\% / 00)\end{array}$ & $\begin{array}{c}\sigma_{\mathrm{t}} \\
(\mathrm{g} / 1)\end{array}$ & $\begin{array}{c}\mathrm{O}_{2} \\
(\mathrm{ml} / 1)\end{array}$ & $\begin{array}{c}\mathrm{PO}_{4}-\mathrm{P} \\
(\mu \mathrm{g} \text { at } / 1)\end{array}$ \\
\hline
\end{tabular}

$\begin{array}{lrrrr}04 & 26.74 & 35.29 & 23.04 & 0.61 \\ 15 & 26.74 & 35.23 & 23.03 & 0.61 \\ 24 & 26.58 & 35.28 & 23.08 & 0.62 \\ 45 & 26.48 & 35.29 & 23.12 & 0.65 \\ 114 & 22.55 & 35.21 & 24.24 & 0.95 \\ 153 & 18.66 & 35.23 & 25.30 & 1.05 \\ 1933 / & 15.24 & 35.10 & 26.01 & 1.27 \\ 195- & & & & \\ 237 & 12.24 & 34.88 & 26.47 & 1.64 \\ 286 & 11.55 & 34.84 & 26.56 & 1.97 \\ 337 & 10.81 & 34.78 & 26.66 & 2.21 \\ 337 & 10.85 & 34.78 & 26.65 & 2.48 \\ 441 \frac{4}{5} & 9.02 & 34.67 & 26.88 & 2.58 \\ 567- & 7.69 & 34.60 & 27.03 & \end{array}$

$1 / 2$ Underway to reduce wire angle.

$\overline{2}$ / Bottle pretripped, but was not recast.

3/ Depth slightly unsure--bottle apparently slipped on wire.

4) Two bottom bottles had slid to end of wire when raised, but values look all right.

STATION 41

$M / V$ Hugh M. Smith: Cruise 14, $1^{\circ} 03^{\prime} \mathrm{N}, 169^{\circ} 00^{\prime} \mathrm{W}$, March 4, 1952. Messenger time: 1448 GCT. Weather: 00, cloud coverage not recorded. Wind: $080^{\circ}, 15 \mathrm{kt}$. Sea: $5-8 \mathrm{ft}$. Wire angle: 1/ $37^{\circ}$

OBSER VED

\begin{tabular}{|c|c|c|c|c|c|}
\hline $\begin{array}{c}\text { DEPTH } \\
(\mathrm{m})\end{array}$ & $\left.{ }^{\mathrm{T}} \mathrm{C}\right)$ & $\left.\%^{\mathrm{S}} / 00\right)$ & $\begin{array}{c}\sigma_{\mathrm{t}} \\
(\mathrm{g} / 1)\end{array}$ & $\begin{array}{c}\mathrm{O}_{2} \\
(\mathrm{ml} / 1)\end{array}$ & $\begin{array}{c}\mathrm{PO}_{4}-\mathrm{P} \\
(\mu \mathrm{g} \text { at } / 1)\end{array}$ \\
\hline
\end{tabular}

$\begin{array}{rrrrr}04 & 26.46 & 35.16 & 23.03 & 0.63 \\ 15 & 26.48 & 35.17 & 23.03 & 0.61 \\ 30 & 26.44 & 35.16 & 23.04 & 0.60 \\ 59 & 26.18 & 35.19 & 23.14 & 0.66 \\ 113 & 25.04 & 35.16 & 23.47 & 0.79 \\ 167 & 16.12 & 34.97 & 25.72 & 1.20 \\ 222 & 12.02 & 34.85 & 26.49 & 1.71 \\ 2752 / & 11.42 & 34.80 & 26.56 & 1.93 \\ 288- & & & & 2.13 \\ 331 & 10.25 & 34.73 & 26.72 & 2.28 \\ 392 & 9.88 & 34.70 & 26.75 & 2.35 \\ 451 & 9.07 & 34.66 & 26.86 & \\ 451 & 9.12 & 34.66 & 26.85 & 2.54 \\ 565 & 7.26 & 34.58 & 27.07 & 2.68 \\ 694 & 5.99 & 34.52 & 27.20 & \end{array}$

1/ Underway to reduce wire angle.

2/ Bottle slipped on wire, depth unsure. Used $275 \mathrm{~m}$. for drawing temperature curve, but may be wrong. 
STATION 42

M/V Hugh M. Smith: Cruise 14, $2^{\circ} 06^{\prime} \mathrm{N}, 168^{\circ} 57^{\prime} \mathrm{W}$, March 5, 1952. Messenger time: first cast $0043 \mathrm{GCT}$, second cast $0144 \mathrm{GCT}$, third cast $0245 \mathrm{GCT}$. Weather: 02 , cloud coverage 2 . Wind: $090^{\circ}, 16 \mathrm{kt}$. Sea: $3-5 \mathrm{ft}$. Wire angle: $1 /$ first cast $42^{\circ}$, second cast $32^{\circ}$, third cast $35^{\circ}$ OBSERVED

\begin{tabular}{|c|c|c|c|c|c|}
\hline $\begin{array}{c}\text { DEPTH } \\
(\mathrm{m})\end{array}$ & $\begin{array}{c}\mathrm{T} \\
\left({ }^{\circ} \mathrm{C}\right)\end{array}$ & $\begin{array}{c}\mathrm{S} \\
(\% / \mathrm{oo})\end{array}$ & $\begin{array}{c}\sigma_{\mathrm{t}} \\
(\mathrm{g} / 1)\end{array}$ & $\begin{array}{c}\mathrm{O}_{2} \\
(\mathrm{ml} / 1)\end{array}$ & $\begin{array}{c}\mathrm{PO}_{4}-\mathrm{P} \\
(\mu \mathrm{g} \text { at } / 1)\end{array}$ \\
\hline
\end{tabular}

\begin{tabular}{lllll}
03 & 27.02 & 35.17 & 22.86 & 0.59 \\
14 & 26.94 & 35.17 & 22.88 & 0.59 \\
28 & 26.87 & 35.15 & 22.89 & 0.62 \\
58 & 26.82 & 35.16 & 22.92 & 0.67 \\
114 & 25.08 & 35.10 & 23.41 & 0.84 \\
170 & 14.03 & 34.70 & 25.96 & 1.52 \\
230 & 11.04 & 34.79 & 26.62 & 1.86 \\
285 & 10.72 & 34.78 & 26.67 & 2.04 \\
\hline 394 & 9.81 & 34.71 & 26.78 & 2.29 \\
472 & 9.18 & 34.63 & 26.86 & 2.34 \\
\hline 522 & 8.46 & 34.62 & 26.92 & 2.39 \\
522 & 9.50 & 34.62 & 26.92 & 2.83 \\
603 & 6.74 & 34.55 & 27.12 & 2.85 \\
815 & 5.22 & 34.53 & 27.30 &
\end{tabular}

1) Underway to reduce wire angle.

\section{STATION 43}

M/V Hugh M. Smith: Cruise 14, $3^{\circ} 05^{\prime} \mathrm{N}, 169^{\circ} 00^{\prime} \mathrm{W}$, March 5, 1952. Messenger time: first cast $1140 \mathrm{GCT}$, second cast $1214 \mathrm{GCT}$. Weather: 00, cloud coverage not recorded. Wind: $130^{\circ}, 10 \mathrm{kt}$. Sea: $3-5 \mathrm{ft}$. Wire angle: first cast $42^{\circ}$, second cast $54^{\circ}$

O BSER VED

\begin{tabular}{|c|c|c|c|c|c|}
\hline $\begin{array}{c}\text { DEPTH } \\
(\mathrm{m})\end{array}$ & $\begin{array}{c}\mathrm{T} \\
\left({ }^{\circ} \mathrm{C}\right)\end{array}$ & $\left.{ }^{\mathrm{S}} / \mathrm{oo}\right)$ & $\begin{array}{c}\sigma_{\mathrm{t}} \\
(\mathrm{g} / 1)\end{array}$ & $\begin{array}{c}\mathrm{O}_{2} \\
(\mathrm{ml} / 1)\end{array}$ & $\begin{array}{c}\mathrm{PO}_{4}-\mathrm{P} \\
(\mu \mathrm{g} \text { at } / 1)\end{array}$ \\
\hline
\end{tabular}

\begin{tabular}{lllll}
60 & 27.16 & 35.11 & 22.77 & 0.63 \\
13 & 27.18 & 35.10 & 22.76 & 0.58 \\
27 & 27.16 & 35.12 & 22.78 & 0.61 \\
55 & 27.08 & 35.12 & 22.81 & 0.64 \\
99 & 27.03 & 35.14 & 22.83 & 0.68 \\
137 & 26.81 & 35.16 & 22.92 & 0.70 \\
173 & 16.07 & 34.63 & 25.51 & 1.32 \\
173 & & & & 1.38 \\
208 & 11.20 & 34.56 & 26.42 & 2.03 \\
\hline 225 & 10.92 & 34.61 & 26.51 & 1.97 \\
263 & 9.91 & 34.63 & 26.70 & 2.27 \\
304 & 9.73 & 34.70 & 26.78 & 2.38 \\
304 & 9.76 & 34.70 & 26.77 & 2.41 \\
403 & 8.70 & 34.63 & 26.89 & 2.56 \\
527 & 7.56 & 34.59 & 27.04 &
\end{tabular}


STATION 44

M/V Hugh M. Smith: Cruise 14, $4^{\circ} 06^{\prime} \mathrm{N}, 168^{\circ} 57^{\prime} \mathrm{W}$, March 5, 1952. Messenger time: 2224 GCT. Weather: 02 , cloud coverage 9.

Wind: $040^{\circ}, 19 \mathrm{kt}$. Sea: $5-8 \mathrm{ft}$. Wire angle: $28^{\circ}$

O B S E R E D

\begin{tabular}{|c|c|c|c|c|c|}
\hline $\begin{array}{c}\text { DEPTH } \\
(\mathrm{m})\end{array}$ & $\begin{array}{c}\mathrm{T} \\
\left({ }^{\circ} \mathrm{C}\right)\end{array}$ & $\begin{array}{c}\mathrm{S} \\
(\% / 00)\end{array}$ & $\begin{array}{c}\sigma_{\mathrm{t}} \\
(\mathrm{g} / 1)\end{array}$ & $\begin{array}{c}\mathrm{O}_{2} \\
(\mathrm{ml} / 1)\end{array}$ & $\begin{array}{c}\mathrm{PO}_{4}-\mathrm{P} \\
(\mu \mathrm{g} \text { at } / 1)\end{array}$ \\
\hline
\end{tabular}

$\begin{array}{rrrrr}00 & 27.72 & 35.05 & 22.54 & 0.51 \\ 13 & 27.74 & 35.07 & 22.55 & 0.53 \\ 31 & 27.69 & 35.07 & 22.57 & 0.53 \\ 66 & 27.65 & 35.09 & 22.60 & 0.57 \\ 132 & 26.90 & 35.12 & 22.86 & 0.55 \\ 198 & 13.30 & 34.59 & 26.03 & 1.83 \\ 254 & 9.87 & 34.63 & 26.70 & 2.38 \\ 327 & 9.10 & 34.60 & 26.81 & 2.34 \\ 395 & 8.51 & 34.60 & 26.90 & 2.37 \\ 469 & 7.94 & 34.56 & 26.96 & 2.48 \\ 538 & 7.39 & 34.55 & 27.03 & 2.90 \\ 538 & 7.44 & 34.55 & 27.02 & 2.90 \\ 678 & 6.22 & 34.50 & 27.15 & 2.89 \\ 331 & 5.36 & 34.51 & 27.27 & \end{array}$

\section{STATION 45}

M/V Hugh M. Smith: Cruise 14, $4^{\circ} 59^{\prime} \mathrm{N}, 168^{\circ} 56^{\prime} \mathrm{W}$, March 6, 1952. Messenger time: $0611 \mathrm{GCT}$. Weather: 02 , cloud coverage 9 . Wind: $050^{\circ}, 18 \mathrm{kt}$. Sea: $5-8 \mathrm{ft}$. Wire angle: $25^{\circ}$

OBSER V E D

\begin{tabular}{|c|c|c|c|c|c|}
\hline $\begin{array}{c}\text { DEPTH } \\
(\mathrm{m})\end{array}$ & $\begin{array}{c}\mathrm{T} \\
\left({ }^{\circ} \mathrm{C}\right)\end{array}$ & $\begin{array}{c}\mathrm{S} \\
\left({ }^{\circ} / 00\right)\end{array}$ & $\begin{array}{c}\sigma_{\mathrm{t}} \\
(\mathrm{g} / 1)\end{array}$ & $\begin{array}{c}\mathrm{O}_{2} \\
(\mathrm{ml} / 1)\end{array}$ & $\begin{array}{c}\mathrm{PO}_{4}-\mathrm{P} \\
(\mu \mathrm{g} \text { at } / 1)\end{array}$ \\
\hline
\end{tabular}

$\begin{array}{lllll}01 & 27.91 & 34.85 & 22.33 & 0.49 \\ 09 & 27.94 & 34.85 & 22.32 & 0.49 \\ 26 & 27.91 & 34.85 & 22.33 & 0.54 \\ 53 & 28.01 & 35.04 & 22.44 & 0.55 \\ 105 & 26.91 & 35.07 & 22.82 & 0.59 \\ 157 & 20.84 & 34.88 & 24.46 & 0.83 \\ 209 & 11.46 & 34.54 & 26.35 & 2.04 \\ 261 & 9.30 & 34.66 & 26.82 & 2.10 \\ 314 & 8.90 & 34.69 & 26.91 & 2.26 \\ 368 & 8.46 & 34.60 & 26.91 & 2.39 \\ 417 & 8.02 & 34.60 & 26.98 & 2.39 \\ 417 & 8.07 & 34.60 & 26.97 & 2.80 \\ 525 & 7.28 & 34.60 & 27.09 & 2.82 \\ 630 & 6.52 & 34.54 & 27.14 & \end{array}$


STATION 46

M/V Hugh M. Smith: Cruise 14, $5^{\circ} 52^{\prime} \mathrm{N}, 169^{\circ} 00^{\prime} \mathrm{W}$, March 6, 1952. Messenger time: $1514 \mathrm{GCT}$. Weather: 00 , cloud coverage not recorded. Wind: $080^{\circ}, 19 \mathrm{kt}$. Sea: 5-8 ft. Wire angle: $08^{\circ}$

OBSER V E D

\begin{tabular}{|c|c|c|c|c|c|}
\hline $\begin{array}{c}\text { DEPTH } \\
(\mathrm{m})\end{array}$ & $\begin{array}{c}\mathrm{T} \\
\left({ }^{\circ} \mathrm{C}\right)\end{array}$ & $\begin{array}{c}\mathrm{S} \\
(\% / \mathrm{oo})\end{array}$ & $\begin{array}{c}\sigma_{\mathrm{t}} \\
(\mathrm{g} / 1)\end{array}$ & $\begin{array}{c}\mathrm{O}_{2} \\
(\mathrm{ml} / 1)\end{array}$ & $\begin{array}{c}\mathrm{PO}_{4}-\mathrm{P} \\
(\mu \mathrm{g} \text { at } / 1)\end{array}$ \\
\hline
\end{tabular}

$\begin{array}{lllll}00 & 27.92 & 34.97 & 22.42 & 0.54 \\ 11 & 27.94 & 34.98 & 22.42 & 0.49 \\ 31 & 27.91 & 34.96 & 22.41 & 0.53 \\ 60 & 27.93 & 34.97 & 22.42 & 0.55 \\ 116 & 23.62 & 34.91 & 23.71 & 0.64 \\ 177 & 15.08 & 34.59 & 25.66 & 1.63 \\ 235 & 10.48 & 34.61 & 26.58 & 2.51 \\ 293 & 9.40 & 34.63 & 26.78 & 2.56 \\ 351 & 8.76 & 34.60 & 26.86 & 2.40 \\ 409 & 8.37 & 34.58 & 26.91 & 2.47 \\ 462 & 7.95 & 34.57 & 26.97 & 2.60 \\ 462 & 7.99 & 34.57 & 26.96 & \\ 578 & 7.12 & 34.53 & 27.05 & 3.08 \\ 690 & 6.08 & 34.51 & 27.18 & \end{array}$

\section{STATION 47}

M/V Hugh M. Smith: Cruise 14, $6^{\circ} 58^{\prime} \mathrm{N}, 168^{\circ} 54^{\prime} \mathrm{W}$, March 7, 1952. Messenger time: 0442 GCT. Weather: 21 , cloud coverage 10. Wind: $080^{\circ}, 16 \mathrm{kt}$. Sea: $8-12 \mathrm{ft}$. Wire angle: $00^{\circ}$

OBSERVED

\begin{tabular}{|c|c|c|c|c|c|}
\hline $\begin{array}{c}\text { DEPTH } \\
(\mathrm{m})\end{array}$ & $\begin{array}{c}{ }^{\mathrm{T}} \\
\left.{ }^{\mathrm{C}} \mathrm{C}\right)\end{array}$ & $\begin{array}{c}\mathrm{S} \\
\left({ }^{\circ} / \mathrm{oo}\right)\end{array}$ & $\begin{array}{c}\sigma_{\mathrm{t}} \\
(\mathrm{g} / 1)\end{array}$ & $\begin{array}{c}\mathrm{O}_{2} \\
(\mathrm{~m} / / 1)\end{array}$ & $\begin{array}{c}\mathrm{PO}_{4}-\mathrm{P} \\
(\mu \mathrm{g} \text { at } / 1)\end{array}$ \\
\hline
\end{tabular}

$\begin{array}{rrrrr}03 & 27.85 & 34.90 & 22.39 & 0.50 \\ 13 & 27.88 & 34.88 & 22.36 & 0.47 \\ 32 & 27.89 & 34.97 & 22.43 & 0.49 \\ 63 & 27.88 & 34.95 & 22.42 & 0.53 \\ 122 & 23.26 & 34.96 & 23.85 & 0.67 \\ 180 & 14.33 & 34.63 & 25.85 & 1.67 \\ 233 & 10.58 & 34.69 & 26.63 & 2.54 \\ 298 & 9.52 & 34.69 & 26.81 & 2.59 \\ 357 & 8.92 & 34.66 & 26.89 & 2.67 \\ 417 & 8.56 & 34.67 & 26.95 & 2.70 \\ 471 & 8.17 & 34.61 & 26.96 & 2.75 \\ 471 & 8.21 & 34.61 & 26.95 & 2.96 \\ 588 & 7.40 & 34.55 & 27.03 & 3.01 \\ 701 & 6.55 & 34.52 & 27.12 & \end{array}$


